\title{
THE PALOMAR TRANSIENT FACTORY ORION PROJECT: ECLIPSING BINARIES AND YOUNG STELLAR OBJECTS
}

\author{
Julian C. van Eyken ${ }^{1}$, David R. Ciardi ${ }^{1}$, Luisa M. Rebull ${ }^{2}$, John R. Stauffer ${ }^{2}$, Rachel L. Akeson ${ }^{1}$, \\ Charles A. Beichman ${ }^{1}$, Andrew F. Boden ${ }^{3}$, Kaspar von Braun ${ }^{1}$, Dawn M. Gelino ${ }^{1}$, D. W. Hoard ${ }^{2}$, Steve B. Howell , $^{4}, 5$, \\ Stephen R. Kane ${ }^{1}$, Peter Plavchan ${ }^{1}$, Solange V. Ramírez ${ }^{1}$, Joshua S. Bloom ${ }^{6}$, S. Bradley Cenko ${ }^{6}$, Mansi M. Kasliwal ${ }^{7}$, \\ Shrinivas R. Kulkarni ${ }^{7}$, Nicholas M. LaW ${ }^{8}$, Peter E. Nugent ${ }^{9}$, Eran O. OfeK ${ }^{7}, 13$, Dovi Poznanski ${ }^{6,9,13}$, \\ Robert M. Quimby ${ }^{7}$, Carl J. Grillmair ${ }^{10}$, Russ Laher ${ }^{10}$, David Levitan ${ }^{11}$, Sean Mattingly ${ }^{12}$, And Jason A. Surace ${ }^{10}$ \\ ${ }^{1}$ NASA Exoplanet Science Institute, California Institute of Technology, Pasadena, CA 91125, USA; vaneyken@ipac.caltech.edu \\ ${ }^{2}$ Spitzer Science Center/Caltech, M/S 220-6, Pasadena, CA 91125, USA \\ ${ }^{3}$ Caltech Optical Observatories, California Institute of Technology, Pasadena, CA 91125, USA \\ ${ }^{4}$ National Optical Astronomy Observatory, Tucson, AZ 85719, USA \\ ${ }^{5}$ NASA Ames Research Center, M/S 244-30, Moffett Field, CA 94035, USA \\ ${ }^{6}$ Department of Astronomy, University of California, Berkeley, CA 94720-3411, USA \\ ${ }^{7}$ Cahill Center for Astrophysics, California Institute of Technology, Pasadena, CA 91125, USA \\ ${ }^{8}$ Dunlap Institute for Astronomy and Astrophysics, University of Toronto, Toronto M5S 3H4, Ontario, Canada \\ ${ }^{9}$ Computational Cosmology Center, Lawrence Berkeley National Laboratory, Berkeley, CA 94720, USA \\ ${ }^{10}$ Spitzer Science Center, M/S 220-6, California Institute of Technology, Jet Propulsion Laboratory, Pasadena, CA 91125, USA \\ ${ }^{11}$ Department of Physics, California Institute of Technology, Pasadena, CA 91125, USA \\ ${ }^{12}$ Department of Physics and Astronomy, The University of Iowa, Iowa City, IA 52242, USA \\ Received 2011 March 30; accepted 2011 June 12; published 2011 July 15
}

\begin{abstract}
The Palomar Transient Factory (PTF) Orion project is one of the experiments within the broader PTF survey, a systematic automated exploration of the sky for optical transients. Taking advantage of the wide $(3.5 \times 2.3)$ field of view available using the PTF camera installed at the Palomar 48 inch telescope, 40 nights were dedicated in 2009 December to 2010 January to perform continuous high-cadence differential photometry on a single field containing the young (7-10 Myr) 25 Ori association. Little is known empirically about the formation of planets at these young ages, and the primary motivation for the project is to search for planets around young stars in this region. The unique data set also provides for much ancillary science. In this first paper, we describe the survey and the data reduction pipeline, and present some initial results from an inspection of the most clearly varying stars relating to two of the ancillary science objectives: detection of eclipsing binaries and young stellar objects. We find 82 new eclipsing binary systems, 9 of which are good candidate 25 Ori or Orion OB1a association members. Of these, two are potential young W UMa type systems. We report on the possible low-mass (M-dwarf primary) eclipsing systems in the sample, which include six of the candidate young systems. Forty-five of the binary systems are close (mainly contact) systems, and one of these shows an orbital period among the shortest known for W UMa binaries, at $0.2156509 \pm 0.0000071$ days, with flat-bottomed primary eclipses, and a derived distance that appears consistent with membership in the general Orion association. One of the candidate young systems presents an unusual light curve, perhaps representing a semi-detached binary system with an inflated low-mass primary or a star with a warped disk, and may represent an additional young Orion member. Finally, we identify 14 probable new classical T-Tauri stars in our data, along with one previously known (CVSO 35) and one previously reported as a candidate weak-line T-Tauri star (SDSS J052700.12+010136.8).
\end{abstract}

Key words: binaries: close - binaries: eclipsing - open clusters and associations: individual (25 Ori) - planets and satellites: detection - stars: pre-main sequence - techniques: photometric

Online-only material: color figures

\section{INTRODUCTION}

The Palomar Transient Factory (PTF) is a survey built around a wide-field mosaic camera installed on the Palomar 48 inch Samuel Oschin telescope dedicated to surveying the sky to find photometric transient and variable objects with variability timescales of minutes to years. The camera consists of a mosaic of two rows of six $2 \mathrm{k} \times 4 \mathrm{k}$ CCDs (of which one is non-functional), with $1^{\prime \prime}$ pixels, giving a total field size of $\approx 3^{\circ} .5 \times 2.3$. With a $5 \sigma$ detection limit of $R \approx 21.0 \mathrm{mag}$ for $60 \mathrm{~s}$ exposures (Law et al. 2010), the main survey is fully automated, and is designed to allow for very rapid follow-up of transient events using the Palomar 60 inch telescope as well as a further

\footnotetext{
${ }^{13}$ Einstein Fellow.
}

global network of telescopes for additional characterization. A technical summary of the system is given by Law et al. (2009) and an overview of the science goals is given by Rau et al. (2009). A summary of the first year's performance is given by Law et al. (2010).

Among the programs within the survey is the PTF Orion project. The primary goal of the Orion project is to search for transiting exoplanets around stars that are 5-10 Myr old. Little is known empirically about exoplanets during the first few millions of years of their lives - the timescales on which they are expected to form and migrate - and the aim is to try and fill this gap in order to provide constraints on theories of planet formation and evolution (e.g., Armitage 2009). Typical young circumstellar disk lifetimes are on the order of 5-10 Myr (Hillenbrand 2008), and by trying to catch stars at or just after 
the point of disk dissipation, we can hope to find planets at the point where they may first become observable without their photometric signatures being swamped by that of the disk, and where disk-planet interaction has ceased.

In addition to the planet search, the observations provide a unique data set to study a variety of stellar astrophysics: eclipsing binary systems enable tests of star formation and evolution models, stellar activity and rotation at these young ages can be characterized, and previously unknown pre-mainsequence (PMS) stars can be identified and characterized within the region. (A similar young transit and eclipse search, the Monitor project, is described by Aigrain et al. 2007 and Miller et al. 2008.)

The 25 Ori association, with an estimated age of around 7-10 Myr (Briceño et al. 2007), provides an ideal target to achieve the goals of the PTF Orion survey, being the most populous known sample in its age range within $500 \mathrm{pc}$, the right age, and with a spatial extent well matched to the PTF field of view. The field has already been well studied and characterized by Briceño et al. (2005, 2007). In the winter of 2009/2010, we obtained time series data centered on the 25 Ori association, taking continuous $R$-band observations and obtaining $\approx 110,000$ individual high-cadence light curves.

The unique capacity of eclipsing binary systems for yielding accurate measurements of fundamental stellar parameters, such as mass, radius, and effective temperature ratio, makes them useful for constraining stellar models. This is especially true of young PMS systems, where evolutionary models are not as well constrained observationally (Mathieu et al. 2007). At primary masses below $1.5 M_{\odot}$, Hebb et al. (2010) list in addition to their newly reported system, MML 53, only six known PMS eclipsing binaries (RXJ 0529.4+0041A, Covino et al. 2000, 2004; V1174 Ori, Stassun et al. 2004; 2MASS J05352184-0546085, Stassun et al. 2006, 2007; JW 380, Irwin et al. 2007; Par 1802, Cargile et al. 2008; Stassun et al. 2008; and ASAS J052821+0338.5, Stempels et al. 2008), with a further four candidates in the Orion Nebula cluster more recently reported by Morales-Calderón et al. (2011). Evolutionary models for stars at the late end of the stellar mass range ( $\gtrsim \mathrm{M} 0)$ are also currently not well constrained by empirical measurements, owing to their intrinsic faintness, and the consequent difficulty in observing them. To date, fewer than 20 such systems are known (see Kraus et al. 2011 , and references therein). It is therefore of particular interest to identify binary systems that are young Orion members and/or that lie in the low-mass regime in our data set.

Observations of contact binary systems, commonly known as W UMa systems, are also of interest both because they are potentially useful distance indicators (Rucinski 2004; Eker et al. 2009) and because their formation and evolution is currently not well understood (see, e.g., Bilir et al. 2005; Eker et al. 2006; Pribulla \& Rucinski 2006). It has been debated whether they may form as the result of the merger of previously detached binary systems through angular momentum loss due to tidal interaction and magnetic braking (e.g., Stępień 1995, 2006) or as a result of initially detached binary orbits decaying by a combination of the Kozai mechanism and tidal friction due to the presence of a third more distant body (Tokovinin et al. 2006; Rucinski et al. 2007; Eggleton \& Kisseleva-Eggleton 2006) or instead directly as contact systems (e.g., Bilir et al. 2005; Roxburgh 1966). The 7-10 Myr age of the 25 Ori region, for example, is too short for the expected Gyr timescale needed for in-spiral via angular momentum loss (Stępień 1995; 2006; Bilir et al. 2005; Gazeas \& Stępień 2008), and indeed, appears somewhat short even for a merger by the Kozai/tidal-friction mechanism, which operates on timescales of tens of Myr or more (Eggleton $\&$ Kisseleva-Eggleton 2006). Finding young contact binaries within our sample is potentially useful for constraining these formation mechanisms.

Single PMS T-Tauri stars (TTSs) are also useful for understanding stellar formation and early evolution. At the age of the 25 Ori association, they are relatively unobscured and can be observed in the optical regime. The advent of wide-field surveys like the CIDA-QUEST survey (Briceño et al. 2005) allows for broad, deep searches that are sensitive to the more dispersed and more mature low-mass populations, where previous studies have been more tightly focused on the brighter OB stars in relatively compact regions. The PTF survey is similarly sensitive, and identification of new young TTSs in our data complements the sample reported by Briceño et al. (2005, 2007).

In this first paper, we present a description of the survey and the data reduction procedure, and some of the initial results that relate to these two stellar astrophysical science objectives of the program: binary systems and new candidate PMS stars. Section 2 describes the survey; Section 3 describes the data reduction pipeline developed for the PTF Orion project; Section 4 gives an overview of the data obtained and an assessment of the current precision levels; Section 5 discusses the eclipsing binaries found including the candidate young binary systems, the low-mass binaries, and some of the contact and near-contact binaries of interest (with some overlap between these groups); and Section 6 discusses the new classical T-Tauri stars (CTTSs). A brief summary is given in Section 7.

\section{THE PTF ORION SURVEY}

\subsection{Field Selection}

Our target field around 25 Ori was chosen to balance a number of requirements. Interstellar reddening is minimal along the line of sight to the cluster (mean $A_{V}=0.29$; Briceño et al. 2007); the source count density is optimal, being high but without overcrowding $(\approx 4.4$ sources per square arcminute at our detection threshold); and the region is already relatively well characterized, with an estimated age of $\approx 7-10 \mathrm{Myr}$ (Briceño et al. 2007), corresponding to the time at which the disks around young stars have mostly dissipated (Hillenbrand 2008). This allows us to probe the photometric variability of young stars without it being completely dominated by variations due to accretion or circumstellar extinction. The majority of TTSs in the 25 Ori region are weak-lined T-Tauri stars (WTTSs), indicating that the disks have indeed dissipated (Briceño et al. 2007).

Within these constraints, the field was aligned to maximize the number of known TTSs covered (Briceño et al. 2005, 2007; C. Briceño 2009, private communication) while allowing for the non-functioning chip, and to place 25 Ori itself on a gap between chips, minimizing charge bleeding from what would otherwise be a heavily saturated source. Figure 1 shows the positioning of the field with respect to 25 Ori and the brighter stars in the region. The field is centered at approximately $\alpha=05^{\mathrm{h}} .26 .46^{\mathrm{s}}$., $\delta=+01.50$ '.50'. (J2000), covering an area 3.5 in right ascension and 2.3 in declination. One of the chips in the array is nonfunctional (the northeast of the central four, chip number 4) and is not shown. 


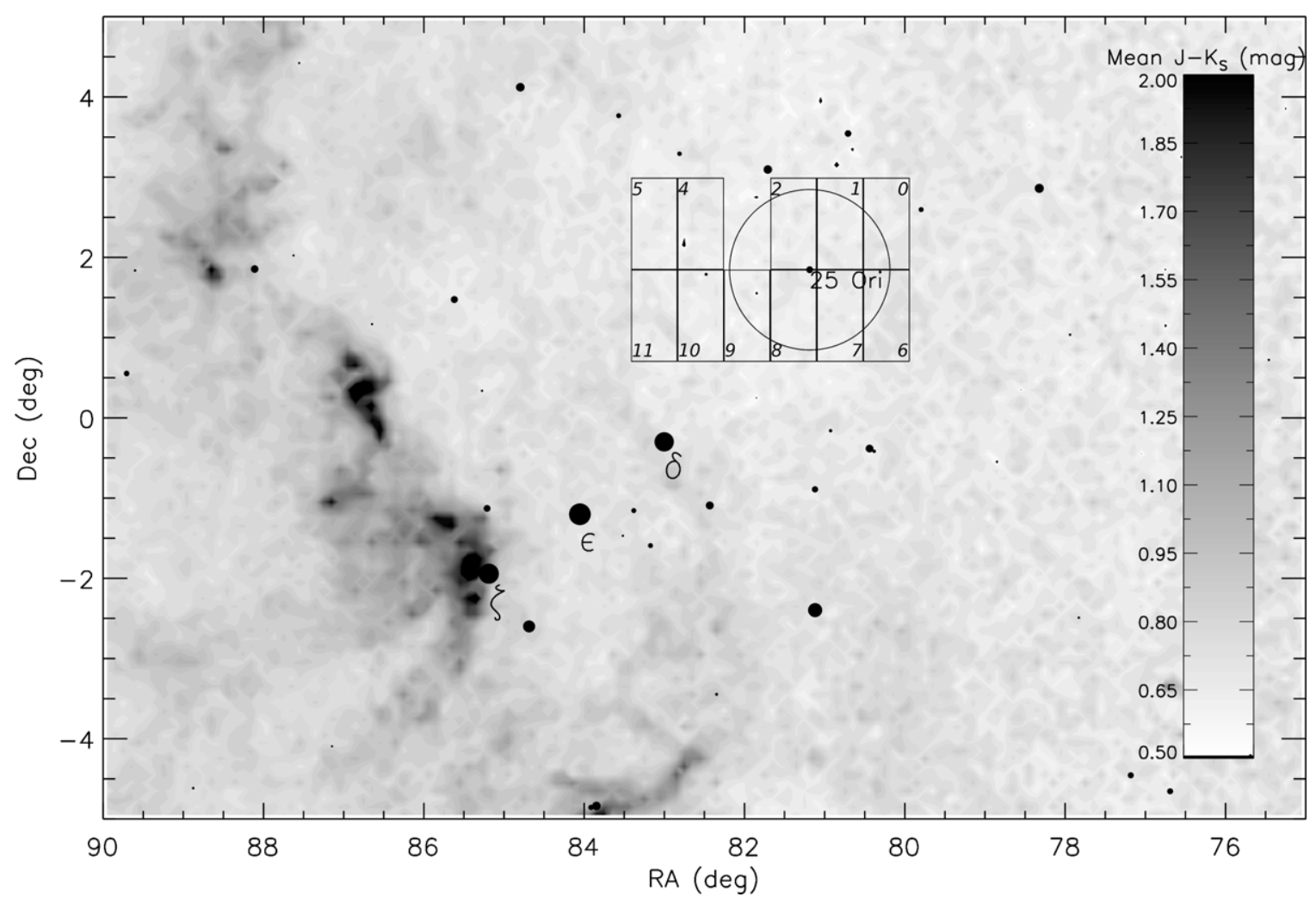

Figure 1. Location of the field with respect to 25 Ori and the Orion belt stars, overlaid on gray-scale contours representing mean 2 MASS $J-K_{s}$ colors, which trace reddening. Bin size is $0.1 \times 0$. 1 . Filled circles mark bright stars $(V \leqslant 6.0 \mathrm{mag})$, with size varying linearly with visual magnitude; the brightest of these are labeled, along with 25 Ori which is placed in a chip gap in the PTF field of view. The footprint for each chip in the field is marked and the chip number is identified; the dead chip (NE of the central four) is omitted. The circle shows the $1^{\circ}$ working radius used by Briceño et al. (2007) to define the 25 Ori group.

The figure confirms that the amount of interstellar reddening is apparently uniformly quite low in the 25 Ori field, and we adopt the derived mean extinction value for the cluster from Briceño et al. (2007; $\left.A_{V}=0.29 \mathrm{mag}\right)$ as a single representative value. (In comparison, typical values from the dust maps of Schlegel et al. 1998 along lines of sight to the sources in the field range from $\approx 0.3$ to $0.6 \mathrm{mag}$, and mostly $<1.0 \mathrm{mag}$. As estimates of the full line-of-sight extinction through to the edge of the Galaxy, these suggest an upper limit.) Following the extinction laws of Cardelli et al. (1989), and assuming $R_{V}=3.1$, the adopted value of $A_{V}$ corresponds to extinctions in $R, J, H$, and $K_{S}$ of $0.22 \mathrm{mag}, 0.082 \mathrm{mag}, 0.055 \mathrm{mag}$, and 0.033 mag, respectively.

\subsection{PTF Observations}

Data were taken for the Orion project on the majority of the clear nights between 2009 December 1 and 2010 January 15. Weather permitting, the chosen primary field was observed in the $R$ filter as near to continuously as possible, whenever it was higher in the sky than an airmass of 2.0. Exposures were 30 s long, with a cadence varying generally between 70 and $90 \mathrm{~s}$, including readout time and depending on the performance of the telescope control system and guiding control loop (see below).

Since the Orion observing program could not easily be incorporated into the normal PTF robotic scheduling, separate scripts for the telescope and camera control code were adapted from the standard PTF robot code for the purposes of the Orion observations. Each night at the beginning of the Orion field observations, normal PTF operations were manually interrupted to run the Orion observing program. When the field center sets once again below airmass 2.0, control was automatically returned to the standard PTF robot scheduler and normal PTF operations resumed.

Of the 40 nights dedicated to the Orion project, 14 yielded usable data, the majority of the remainder being either of marginal quality due to clouds or subject to telescope closure because of poor weather. (One night also could not be processed due to an insufficiency of non-Orion exposures to create a flat field for that night, and can in principle be recovered.) A total of 4486 exposures were taken during the run. An average across the chips of $\approx 3460$ exposures ran successfully through the pipeline after rejection of those that could be immediately discarded at the outset by visual inspection, and an average of $\approx 2400$ of those exposures passed the data reduction pipeline's automated data quality assessment without flagging. There is some variation in these numbers from chip to chip since each is processed completely independently. Figure 2 shows a histogram of the number of exposures taken on each night, and the fraction of those that passed unflagged for chip 0 , which is used as a representative example of all the chips.

In order to minimize the effects of flat-fielding errors for highprecision photometry, it is desirable to minimize image shift on the detector between frames. Since the Palomar 48 inch has no native guiding facility, we developed software to guide on the science images as they came from the telescope, providing feedback to the telescope via offset pointing commands. Offsets were referenced to a single good quality reference frame taken at the beginning of the run, and telescope pointing updates were applied during readout time generally every second frame. On initial pointing of the telescope, offsets of typically $\approx 70-90$ pixels relative to the reference frame were normally 


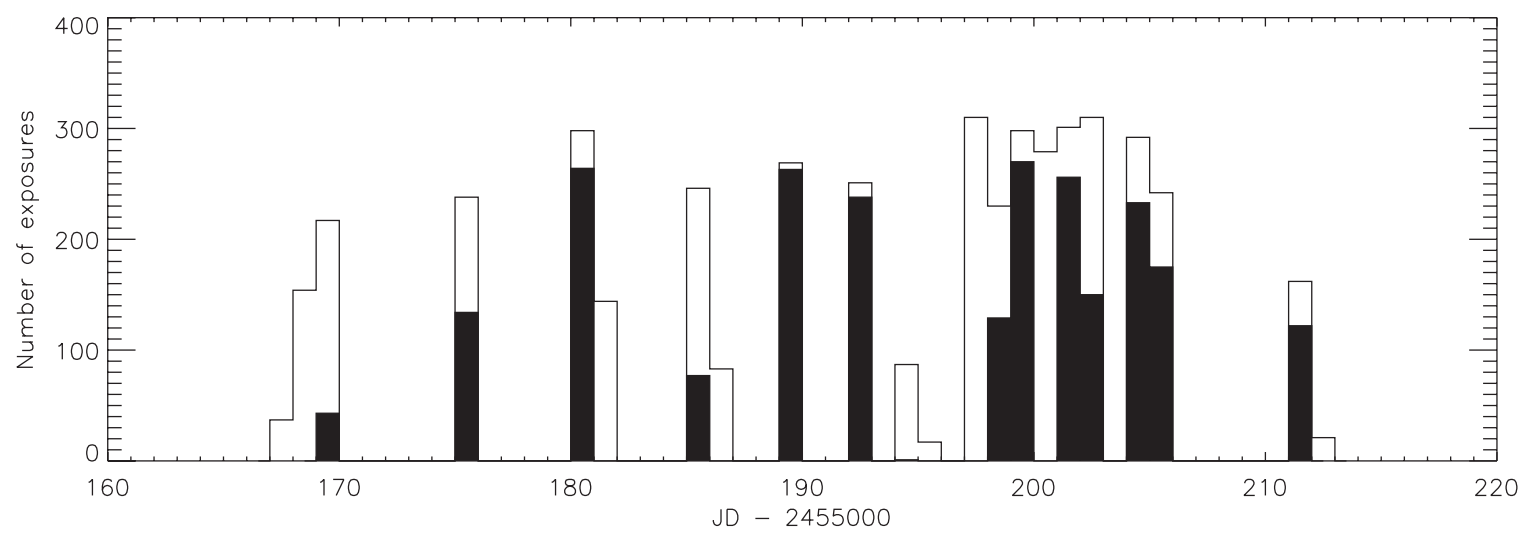

Figure 2. Number of exposures taken per night for the PTF Orion project from 2009 December to 2010 January. Solid black regions indicate the fraction of exposures that remained unflagged after processing by the data reduction pipeline, for chip 0 (similar fractions are seen for the other chips).

corrected within two exposures. With the guiding switched on, the pointing toward the center of the field was stabilized with a root-mean-square $(\mathrm{rms})$ image shift of $\approx 1.0$ and $\approx 0.5$ pixels in right ascension and declination, respectively, compared to unguided linear tracking drifts of $\approx 0.3$ pixel minute ${ }^{-1}$.

\subsection{Ancillary Data}

In addition to the PTF data obtained, we also drew on data from several other sources. The photographic US Naval Observatory USNO-B1.0 source catalog (Monet et al. 2003) provided a reference data set for initial photometric zero-point correction of the $R$-band PTF Orion data (Section 3.1). A further catalog of $\approx 40,000$ reference stars from the Centro de Investigaciones de Astronomía-Quasar Equatorial Survey Team (CIDA-QUEST), provided by C. Briceño (2009, private communication), allowed for a final more accurate correction to the $R$-band zero point after differential photometry had been performed (Section 3.2). This catalog had in turn been calibrated against the Landolt system (Landolt 1992) with $R$ magnitudes ranging from $14 \lesssim R \lesssim 19$ and measurement errors varying with increasing magnitude from $\approx 0.015$ to $0.1 \mathrm{mag}$. The overall error for the brighter stars (down to $R \approx 17$ ) including systematics is $\approx 0.02-0.03 \mathrm{mag}$. We also made use of the PPMXL catalog (Roeser et al. 2010) to obtain proper motions for our candidate binary systems. The PPMXL catalog provides a determination of positions and proper motions by combining USNO-B1.0 and Two Micron All-Sky Survey (2MASS) astrometry, and aims to be completed to $V \approx 20 \mathrm{mag}$.

The 2MASS point-source catalog (Skrutskie et al. 2006) provides all-sky coverage of point sources in the near-infrared $J(1.25 \mu \mathrm{m}), H(1.65 \mu \mathrm{m})$, and $K_{s}(2.16 \mu \mathrm{m})$ bandpasses, with magnitude limits of $15.8,15.1$, and $14.3 \mathrm{mag}$, respectively. PTF Orion source detections were matched against this catalog to provide color information on each source in combination with the PTF Orion $R$ magnitude. The 2MASS catalog includes a "quality" flag for each measurement in each band, which can take various values. In particular, designations from " $\mathrm{A}$ " to " $\mathrm{F}$ " represent photometric quality in decreasing order (in the case of an " $F$," error estimates could not be determined). In certain cases throughout this paper we use these flags to restrict analysis to sources with 2MASS data above a given quality level, as stated in the text.

For certain target objects, anticipating that these young objects might have discernible mid-infrared (MIR) excesses due to circumstellar disks, we also examined the Spitzer Space Telescope (Werner et al. 2004) Heritage Archive for both Infrared
Array Camera (IRAC; Fazio et al. 2004) and Multiband Imaging Photometer for Spitzer (MIPS; Rieke et al. 2004) data, discussed below. We found some IRAC data (at 3.6, 4.5, 5.8, and $8 \mu \mathrm{m}$ ), but MIPS data (at $24 \mu \mathrm{m}$ ) were more common in our target region. We found MIPS and/or IRAC data for 15 objects, discussed in Sections 5.2.5 and 6. All 15 of the objects were found in at least one of 9 AORKEYs ${ }^{14}: 10987776,26066432$, and/ or 26067456 for IRAC, and 10987008, 17052160, 26067712, 26067968, 26069760, and/or 26070016 for MIPS. The images for each object were examined for potential source confusion, and none was found.

For IRAC, we started from the automatically produced mosaics from the Archive (S18.7 or greater), and performed aperture photometry using the IDL ${ }^{15}$ routine aper.pro ${ }^{16}$ on these mosaics at the designated positions. We used an aperture radius of 3 native pixels (1.2 $\operatorname{arcsec}_{\text {pixel }}^{-1}$ ), a sky annulus of radius 3-7 native pixels, and multiplicative aperture corrections of 1.124 , $1.127,1.143$, and 1.234 for the four channels, respectively, as prescribed by the IRAC Instrument Handbook, available on the Spitzer Science Center Web site. The zero points we used to convert between flux density units (Jy) and magnitudes were, respectively, 280.9, 179.7, 115.0, and 64.13 Jy.

For MIPS, we initially obtained the automatically produced mosaics from the Archive (S18.12 for MIPS), and on a case-bycase basis, reprocessed the mosaics if necessary from the basic calibrated data level, to reduce the influence of instrumental artifacts. We also conducted aperture photometry using IDL's aper.pro on the mosaics (either ours or the Archive's), using an aperture of 7 ", an annulus of $7 "-13^{\prime \prime}$, and an aperture correction of 2.05, from the MIPS Instrument Handbook, again available from the SSC Web site. The MIPS-24 zero point we used was $7.14 \mathrm{Jy}$.

In the ideal case, to determine whether or not there is infrared excess due to a circumstellar disk, we need an estimate of a spectral type so that we can fit a model to the spectral energy distribution (SED) and estimate reddening $\left(A_{V}\right)$, and then estimate the degree of infrared excess due to a disk. In the absence of an accurate estimate of spectral type, the degree of IR excess is not well defined. We can instead attempt to estimate the degree of infrared excess by assuming that these sources

\footnotetext{
14 AORKEYs are the unique eight-digit identifier for the Astronomical Observation Request, which can be used to retrieve these data from the Spitzer Heritage Archive.

15 http://www.ittvis.com/idl

16 Found in the IDL Astronomy User's Library, http://idlastro.gsfc.nasa.gov/; see also Landsman (1993) and Stetson (1987).
} 
are all truly stars (as opposed to background objects), and then comparing the longer Spitzer bands to a shorter band. Where possible, even lacking a spectral type estimate, we can use [3.6]- [24] to reasonably reliably indicate disk excess (where the bracket notation denotes the magnitudes at the Spitzer bands), since this range is within the Rayleigh-Jeans tail even for the latest spectral types, giving them colors of zero. Reddening also does not strongly affect this color unless $A_{V}$ is extreme, in which case the extinction will be high enough to make a source detection unlikely in our PTF data. MIPS-24 data are more widely available in our field than the IRAC bands, however, and $3.6 \mu \mathrm{m}$ data do not always exist. In such cases, the 2MASS $K_{s}$ band is the most commonly available alternative. $K_{s}-[24]$ therefore provides an alternative excess estimate, although it is less reliable since late-type stars (such as many of those in our sample) are not colorless at $K_{s}-$ [24] (Gautier et al. 2007), and the effects of reddening are stronger at $K_{s}$ than at $3.6 \mu \mathrm{m}$. For such cases, additional follow-up observations will be needed to refine the estimate of the excess.

\section{THE PTF ORION DATA REDUCTION PIPELINE}

The initial PTF data reduction pipeline in place at the time the data were taken was geared primarily toward absolute photometry and extensive database operations for handling the vast quantities of data coming from the broader survey. We developed a separate dedicated relative-photometry pipeline, which took over from the standard pipeline at the point where image processing was complete. Here, we describe the two broad steps in the data reduction procedure: obtaining the initial raw photometry, beginning with the standard PTF image processing and the first steps of the Orion pipeline, and the differential photometry process used to obtain the precisions needed for the PTF Orion survey.

\subsection{Raw Photometry}

The main PTF pipeline image processing consisted of standard bias subtractions, flat fielding, and astrometric solutions. Each chip was reduced separately and in parallel. Floating biases were measured as the median of the overscan regions of the chips and subtracted along with a nightly combined superbias frame; flat fields were created by combining all (non-Orionproject) images taken with the same filter during a night, all the bright pixels first being masked out using SExtractor (Bertin \& Arnouts 1996), to leave a pure sky flat field. Astrometric information was added to the FITS image headers using SCAMP (Bertin 2006) to solve for the astrometry. The general PTF pipeline is discussed in more detail in Grillmair et al. (2010) and R. Laher et al. (2011, in preparation).

The Orion pipeline ran separately and took as input the image data from the standard pipeline after the astrometric solutions had been added. Written in IDL, the main steps in the PTF Orion pipeline are as follows.

1. Pixel masking. Masks are initially applied to reject any badly behaved pixels on the detector using an algorithm loosely based on the IRAF ${ }^{17}$ "ccdmask" procedure (Tody 1986, 1993). Masks are created from images made by dividing a $70 \mathrm{~s}$ light-emitting diode $\left(\mathrm{LED}^{18}\right)$ flat field by a 35 s LED flat field; three independent such divided frames were obtained for each chip. Any pixels with outlier fluxes

\footnotetext{
17 http://iraf.noao.edu/

18 Light-emitting diode-see Law et al. (2009).
}

beyond $4 \sigma$ in at least two of the three frames, or beyond $3 \sigma$ in all three of the frames were flagged as bad. This approach helps catch excessively variable pixels in addition to nonlinear pixels, while still rejecting cosmic-ray hits. The flagging procedure was then repeated several times after boxcar smoothing of the original image along the readout direction with a selection of bin sizes from 2 to 20 pixels (this removes columns where individual pixels are not statistically bad, but are statistically bad when taken as an aggregate). Pixels lying in small gaps between bad pixels were then also iteratively flagged, with the aim of completely blocking out large regions of bad pixels while minimizing encroachment into good pixel regions. Any source detection with a flagged pixel within its photometry aperture is rejected and does not pass through the pipeline.

2. Raw photometry. Source detection and aperture photometry are performed using the IDL Astronomy User's Library implementation of DAOPHOT (Landsman 1993; Stetson 1987), with minor modifications to return more significant figures in the output files and to handle the large number of sources in each CCD frame. Owing to the sometimes large offsets of the field on the detector from image to image, source detection is performed separately on every exposure obtained, with a $4 \sigma$ detection threshold. A 4 pixel aperture radius provides a reasonable compromise between maximizing the flux enclosed for bright sources and minimizing sky-background noise for faint sources, while also being large enough to prevent significant photometric errors from imperfect centroiding of the apertures. This compares to median seeing of 2".5 at FWHM (where 1 pixel $\approx 1^{\prime \prime}$ ). Inner and outer radii of 10 and 20 pixels, respectively, are chosen for the annulus used for background estimation around the aperture. The default limits for "sharpness" and "roundness" of the detected sources provide a first cut to reject cosmic rays and background galaxy detections.

3. Initial zero-point correction. An initial zero-point correction is applied by matching detections against the USNOB 1.0 catalog and correcting for the outlier-resistant ${ }^{19}$ mean difference between raw and USNO-B magnitudes for each image from each chip. This places all photometry on an absolute scale, within the accuracy of the USNO-B catalog ( $\sim 0.3 \mathrm{mag})$. Sloan Digital Sky Survey (SDSS) photometry data would provide a more accurate correction, but were not available for the 25 Ori field.

4. Creation of master source list. After filtering out images with poor seeing or high background, a master source list for each chip is created by stepping through each of the 100 images with the highest numbers of source counts, in decreasing order of source counts. For each image, if a source is detected whose sky position cannot be matched with one already found (within a $2^{\prime \prime}$ radius, the matching radius used throughout the pipeline), it is added to the master list. On every 20th image, sources with only one detection are pruned from the table, as are any sources with fewer than 10 detections at the end of the process. This ensures that cosmic rays, asteroids, and spurious detections are rejected from the table. Since we are primarily concerned with persistent stellar objects with relatively low-level variability, we accepted the consequent

\footnotetext{
19 Throughout this text, where an outlier-resistant mean or standard deviation is referred to, these are found using the "resistant_mean" and "robust_sigma" functions, respectively, from the IDL Astronomy User's Library.
} 
loss of short transient events that may appear from below the detection limit in return for the significant savings in data storage space in the final output data tables. This does not affect any of the sources that are suitable for planet transit searches.

\subsection{Differential Photometry}

After the initial raw photometry and preparation of the master source lists, a single data table for each chip is prepared with entries for each source in the lists at each epoch of observation. A second pruning of the table is performed to remove sources if they show very few total detections (fewer than 100) and those detections are sparsely distributed in time (the fraction of successful detections within any 50 consecutive epochs is less than 0.5 ). This helps remove sources which are due to badly behaved pixels or on the extreme edge of the detection limit, while retaining genuine transients that are only detected for a limited length of time. The source list from each chip is then split into four equal-area quadrants, each treated separately. This provides a first-order accommodation for variations in photometry across the wide field of view which may be caused by thin cloud, variations in point-spread function (PSF) due to the wide-field optics, effects of differential airmass variation, and atmospheric refraction (see Everett \& Howell 2001). On average $\approx 2600$ sources were detected per chip quadrant for the 25 Ori field.

In a given quadrant, an outlier-resistant mean magnitude is then calculated for each source across all epochs; this creates a reference magnitude list against which to perform the differential photometry. For each observation, the difference is calculated between every source's measured magnitude and its corresponding reference magnitude. Within each quadrant, the mode of the differences is then calculated and subtracted from the measured magnitudes to provide the differentially corrected magnitudes. The mode is calculated by fitting a Gaussian curve to the peak of the histogram of magnitude differences in the quadrant, and the peak of this Gaussian is taken to represent the best-likelihood estimator of the true fine-scale correction for the zero point of the frame. All stars in a given quadrant are thus used in establishing the differential correction applied for that quadrant.

Using the modal magnitude difference correction in this manner has the advantage of being very robust to outliers and variable stars. It also requires no selection of an ensemble set of stable reference stars, provided that there is a sufficient number of stars in the field and that a sufficient number of those stars are relatively stable. Even in the event that only a small fraction of stars are stable, provided that the total number of stars is large, the mode still represents a reasonable estimate of the differential correction since there is no reason to expect any correlation in intrinsic variability between the stars included in the histogram. Additionally, the modal difference draws information from the full ensemble of stars, providing a natural "weighting" of the differences: stars that have higher photometric uncertainties (i.e., lower fluxes) or that are more variable will contribute a more spread-out Gaussian to the total distribution, adding more to the wings of the histogram, though still peaking around the true magnitude difference; those with lower uncertainties (high fluxes) will contribute a tighter Gaussian to the total and contribute more to the peak of the histogram.

To further improve the statistics, the differential photometry is then repeated after identifying and omitting from the histogram sources which still show an rms variation greater than $4 \sigma$ above their mean measurement error, or an rms variation greater than $2 \%$ (or more precisely, an rms greater than the quadrature sum of these two values). This entire procedure is iterated until the total number of sources rejected in this way changes by less than $3 \%$.

As a final step, once differential photometry is complete, the real apparent magnitude is set by comparing the median magnitudes of each differentially corrected time series against the list of stable reference stars from the CIDA-QUEST survey, which in turn are calibrated against the Landolt system (see Section 2.3). The median of the differences is used to provide a final single zero-point correction for each entire chip.

After processing, the data are automatically checked and any questionable sources, nights, or individual data points are flagged according to a number of criteria. Flags are added for failed DAOPHOT photometry or differential photometry; multiple source detections, USNO-B reference sources, or master-list sources, within the FWHM; high image background, very low zero point, or high variation in background across the image; exceptionally large FWHM; low number of sources across the image; large variations with time in the measured right ascension/declination for an individual source location; saturation; high sigma or skew in individual background annuli for sources; proximity to predicted optical ghosts; and indication of crowding or contamination within the photometry aperture (assessed by finding outlier values for the ratio of flux inside the aperture to that in a thin annulus around the aperture compared to other sources in the field).

\section{DATA}

The differential photometry pipeline yielded a total of 116,285 individual light curves (including some resulting from false multiple detections on saturated stars). For an initial investigation of the clearly variable stars, a subsample of all the light curves with an outlier-resistant rms variation greater than three times the outlier-resistant mean measurement error for each light curve was selected. This cut alone yielded $\approx 2060$ sources. These light curves and their corresponding images were visually inspected to select only those that clearly represented intrinsic photometric variation, rather than crowded sources or image artifacts that had escaped flagging, sources on unmasked bad pixels, or sources whose variability was clearly due to known systematics (see Section 4.1). This initial inspection yielded a total of $\approx 530$ sources, the difference in number from the first cut being primarily due to the $\approx 4$ mmag noise floor at the bright end which was not accounted for, and to biasing toward light curves that were not too sparsely sampled. (For comparison, selecting only sources with $>1000$ of a possible $\approx 2400$ unflagged detections, and rms greater than three times the quadrature sum of the mean measurement error and the noise floor, yields $\approx 700$ light curves.) Among the light curves selected from the initial inspection, we visually identified 82 eclipsing binaries and 16 likely CTTS light curves. The results presented in this paper are based on these last two groups of light curves.

\subsection{Precision and Sensitivity}

Figure 3 shows a plot of the standard deviation of unflagged data points versus $R$ magnitude for all of the $\sim 100,000$ light curves obtained, on all 11 chips, over the full length of the observations. The results are compared with the theoretical errors including photon, background, and readout noise. Some chips are better behaved than others, but the noise floor at around 


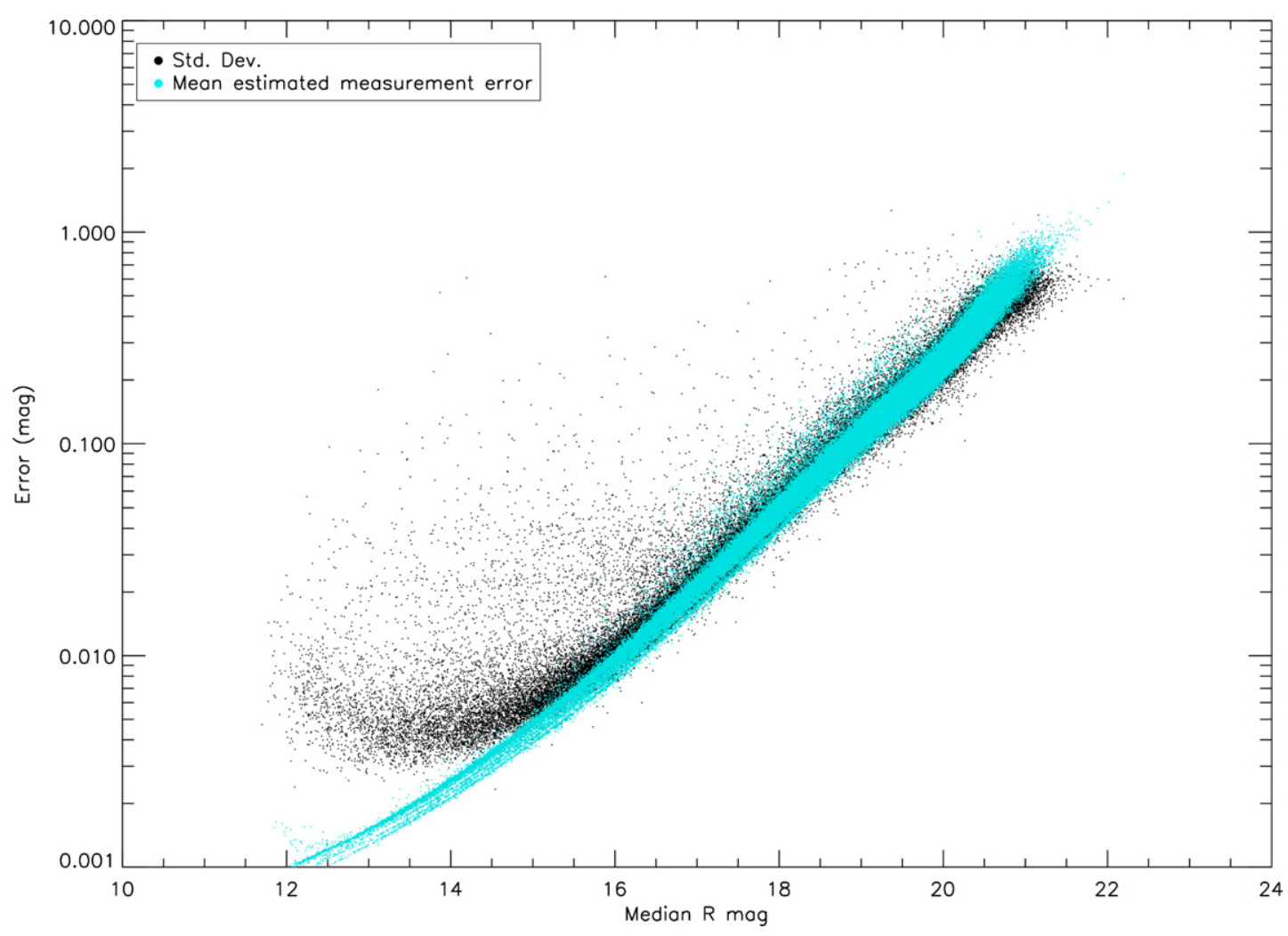

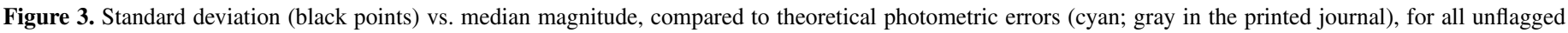

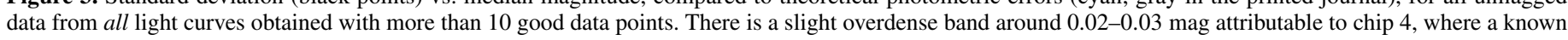
systematic trend affects certain localized regions. See Section 4.1.

(A color version of this figure is available in the online journal.)

4 mmag is evident. Approximately $8 \%$ of all light curves with more than 10 detections have an rms $<1 \%$. The banding in the theoretical errors comes from the different chips and is likely due to small differences in quantum efficiency. Saturation begins to set in around $R \approx 13 \mathrm{mag}$, where the upturn in the rms appears. The faint-end limit varies depending on the chip, but the turn-over in the number of source detections with increasing faintness begins to set in around $R \approx 19.5 \mathrm{mag}$, with few detections beyond 20.5 mag.

In addition to intrinsically variable stars, some of the scatter of points above the noise floor at brighter magnitudes in Figure 3 is due to systematic trends that appear in small regions of certain chips. After performing differential photometry, it is not unusual to discover common systematic trends in some of the resulting light curves that may be a function of position, color, and/or airmass. An advantage of the "modal differencing" technique employed in the differential photometry (Section 3.2) is that, provided the fraction of sources in the ensemble exhibiting a given trend is relatively small, the position of the peak of the difference histogram should be insensitive to the trend. As a result, sources that are not directly affected by the trend will also not be compromised as a result of the differential photometry corrections.

The presence of trends appears to be fairly minimal in the PTF Orion differential photometry, with the exception of one effect that is attributed to known fogging of the detector during the period of the Orion observations, due to slow deposition of oil contaminants from the dry-air system used at the time to prevent condensation on the CCD window (see Law et al. 2010). This contributes a slow long-term trend in certain regions of the detector over the length of the observations, with the exception of the last night, just before which the CCD window had been cleaned. After the cleaning the affected light curves returned to their initial flux level. The trend causes a variation of up to $\approx 0.2$ mag in the worst cases, but is generally easy to recognize, and appears to be confined to relatively small regions on the detector; only a small fraction of the light curves are significantly affected at the level of variability with which we are concerned here. Chip 4 suffered the most from the fogging effect, which leads to a slight overdense band of sources from that chip around 0.02-0.03 mag in Figure 3. At this point light curves that clearly show the trend are rejected from our current analysis for simplicity (four probable eclipsing binaries are affected in this way). A number of sources on chip 4 alone also show random noise on the few percent level on the first three nights and the last night of the run, which may be detector noise related. This should be borne in mind with regard to results from that chip.

There are some suggestions of other small trends, possibly color related, but no detrending of the data has been attempted at this stage, and none of these effects are accounted for in Figure 3. Nonetheless, the figure shows that the majority of the data are well behaved down to the $4 \mathrm{mmag}$ noise floor without any detrending.

\subsection{Absolute Photometric Accuracy}

The absolute accuracy of the magnitudes is dependent on the second zero-point correction against the CIDA-QUEST reference stars (see Section 2.3). The outlier-resistant standard deviation in the magnitude difference between the CIDAQUEST sources and the least variable 50\% of the matched PTF Orion sources ranges from 0.025 to 0.044 mag depending on the chip. Combining this with the overall 0.02-0.03 mag error 
of the CIDA-QUEST list (including systematics), we therefore conservatively estimate the zero points of the individual light curves to be accurate to $\approx 3 \%-5 \%$ for chips $0-4$ and $6-10$.

The area covered by chips 5 and 11 in the Orion field had no coverage in the reference star set, and therefore no direct secondary zero-point correction could be determined. Instead, a correction was made based on the weighted mean of the corrections from the other chips, excluding only chip 4, owing to more significant fogging-trend systematics on that chip which give rise to a distinctly bimodal, non-Gaussian distribution in the zero-point histogram for that chip. The mean of the secondary zero-point corrections for the remaining chips was $0.159 \mathrm{mag}$, with a standard deviation of $0.044 \mathrm{mag}$. Adding this whole-chip zero-point uncertainty in quadrature with the typical uncertainty for the individual light curves discussed above, and a 0.03 mag error for the CIDA-QUEST list, yields an absolute accuracy estimate of 0.069 mag for the light curves on chips 5 and 11.

Marginal color-dependent trends are seen in the magnitude residuals against the CIDA-QUEST references (as a function of $R-I$ ), but they are below the level of the random scatter in the residuals, implying that errors due to mismatch between the PTF and CIDA-QUEST filters are negligible at our accuracy level. Since the error estimates for the zero points are based on the scatter in the residuals about the mean, rather than the error in the mean itself, any systematic errors in zero point should be included in the accuracy estimate.

\section{ECLIPSING BINARY SYSTEMS}

Among the most easily identified of the light curves are the eclipsing binaries. In this section, we present those found in this initial investigation. Section 5.1 presents an overview of the data; an analysis is presented in Section 5.2, where we identify candidate young binaries, and Section 5.3, where we identify some additional low-mass systems. In Section 5.4, we summarize and discuss the specific noteworthy sources.

\subsection{Eclipsing Binary Results}

We identified a total of 82 clear eclipsing binaries in the PTF Orion data, for which period-folded light curves are presented in Figure 4. These were identified by visual inspection of our short list of strongly varying light curves, most eclipsing binary light curve types displaying distinctive characteristic shapes. Light curves that were sinusoidal in appearance were rejected due to the ambiguity in interpretation of their nature. Among the binaries, three were found to have been previously identified as candidate variable stars by Kraus et al. (2007) in the MOTESSGNAT survey (sources 0-9653, 5-12287, and 5-12446). In those cases where a period could not be determined, either because too few eclipses were detected or because the data did not show a regular period, the data covering only the best-captured eclipse are instead plotted against time to show the eclipse shape. The complete light curves for the five such targets are shown in addition in Figure 5.

Table 1 lists the basic properties of the eclipsing binary systems: the assigned PTF Orion survey ID, $N-n$, consisting of the chip number, $N$, on which the source was detected, followed by a running sequence number, $n$; the mean measured $\mathrm{J} 2000$ coordinates over the whole observing run (accurate to $\approx 1$ inch); the corresponding 2MASS ID where a match was obtained (Skrutskie et al. 2006); the 2MASS $J, H$, and $K_{s}$ magnitudes; classification as "close" (C) or "detached" (D) (see below); the period, $P$, in days; $T_{0}$, the heliocentric Julian date for the epoch of the primary eclipse; the estimated distance to the system; the median-measured $R$ magnitude; and $\Delta R$, the approximate peakto-peak magnitude range of the measured light curve. Specific sources discussed in the text are broken out at the beginning of the table. The newly measured properties in the table are obtained as described below.

Classification. The 82 systems are categorized into one of two broad classifications: "close" ("C"), of which we find 45, and "detached" ("D"), of which we find 37. For the purposes of this paper, "close" systems are defined as those for which there is no apparent distinction between in- and out-of-eclipse regions in the light curve, i.e., where there is no clear discontinuity in the slope of the curve. These should represent, for the most part, contact and overcontact binary systems. Those for which such a distinction is apparent are considered "detached," though this category can also include semi-detached and near-contact systems. In some cases semi-detached or near-contact systems may also fall into the "close" category instead, depending on the exact nature of the light curve. We have not attempted to further sub-classify the binary systems to avoid ambiguities that are better addressed with full light curve modeling.

Orbital parameters. Orbital periods, $P$, are obtained by calculating the Plavchan periodogram of the light curve (Plavchan et al. 2008), using a stand-alone version of the NASA/IPAC/ NExScI Star and Exoplanet Database periodogram service. ${ }^{20}$ The light curve is folded on the period corresponding to the highest peak in the periodogram, and visually inspected to confirm the period is reasonable. If not, the next highest peaks are investigated until a plausible folding is found. The error in the period is estimated from the width of a Gaussian fit to the corresponding peak. In cases where the shape of the peak was such that a good Gaussian fit could not be made, the approximate peak was estimated by hand, and an appropriately large error assigned.

The time of mid-eclipse, $T_{0}$, for the systems is measured by fitting a symmetrical inverted trapezium to the deepest and bestsampled minimum of each light curve and calculating the center time of the fitted trapezium. This fit also yields the formal errors for the eclipse center time.

Distance. W UMa systems display a relatively wellestablished empirical period-color-luminosity relationship resulting from their approximately uniform temperature common envelope: Kepler's law constrains the orbital radius as a function of period, and the orbital radius in turn constrains the radiating surface area and, hence, the luminosity and absolute magnitude of the system (Rucinski 2004). They therefore can be used as distance estimators. Eker et al. (2009) provided an updated calibration of the relationship based on 2MASS $\left(J-H\right.$ and $H-K_{S}$ ) colors; we use this relationship to estimate the distances for the "close" binaries in the table where the detections have 2MASS counterparts, on the assumption that the vast majority are $\mathrm{W}$ UMa systems.

Bilir et al. (2008) provided a similar empirical relationship between 2MASS colors and absolute magnitude for mainsequence-detached binaries, analogous to the color-luminosity relationship for single main-sequence stars. We apply this relationship to the "detached" binaries in the table, on the assumption of dwarf star status.

Errors in the derived distances are estimated by standard propagation of the 2MASS photometric errors through the

\footnotetext{
20 http://nsted.ipac.caltech.edu/periodogram/cgi-bin/ Periodogram/nph-simpleupload
} 

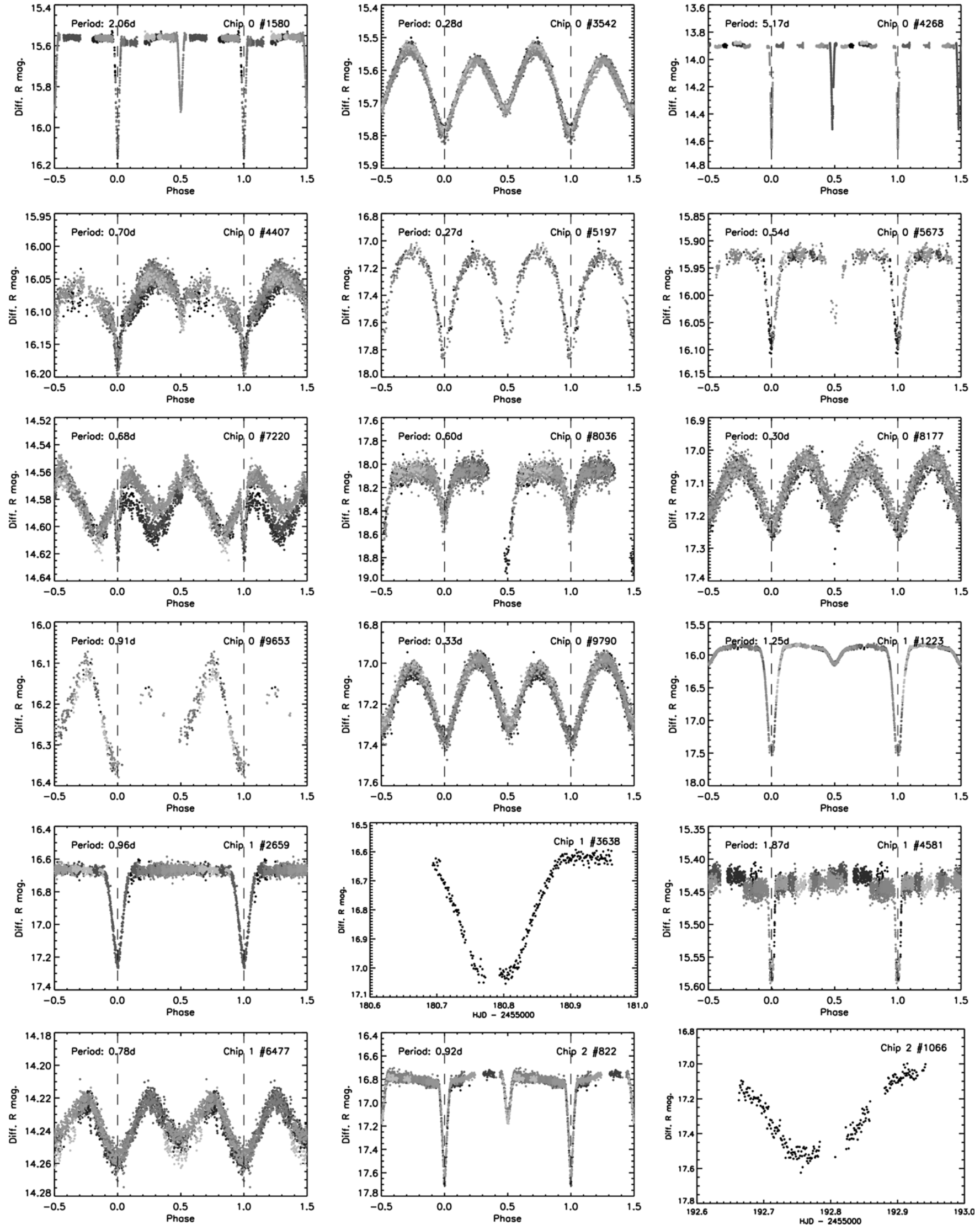

Figure 4. Folded light curves for the eclipsing binaries identified in the data (Section 5.1 and Table 1). The gray scale represents time of observation to give a sense of any long-term variation, running from black at the beginning of the PTF Orion observing run to pale gray at the end. Error bars are omitted for clarity. In cases where insufficient coverage was obtained to determine a period, or where a regular period could not be found, data covering the best eclipse detection are instead plotted in black directly against heliocentric Julian date (complete light curves for these are shown in Figure 5). See Table 1 for the full precision in the measured periods. 

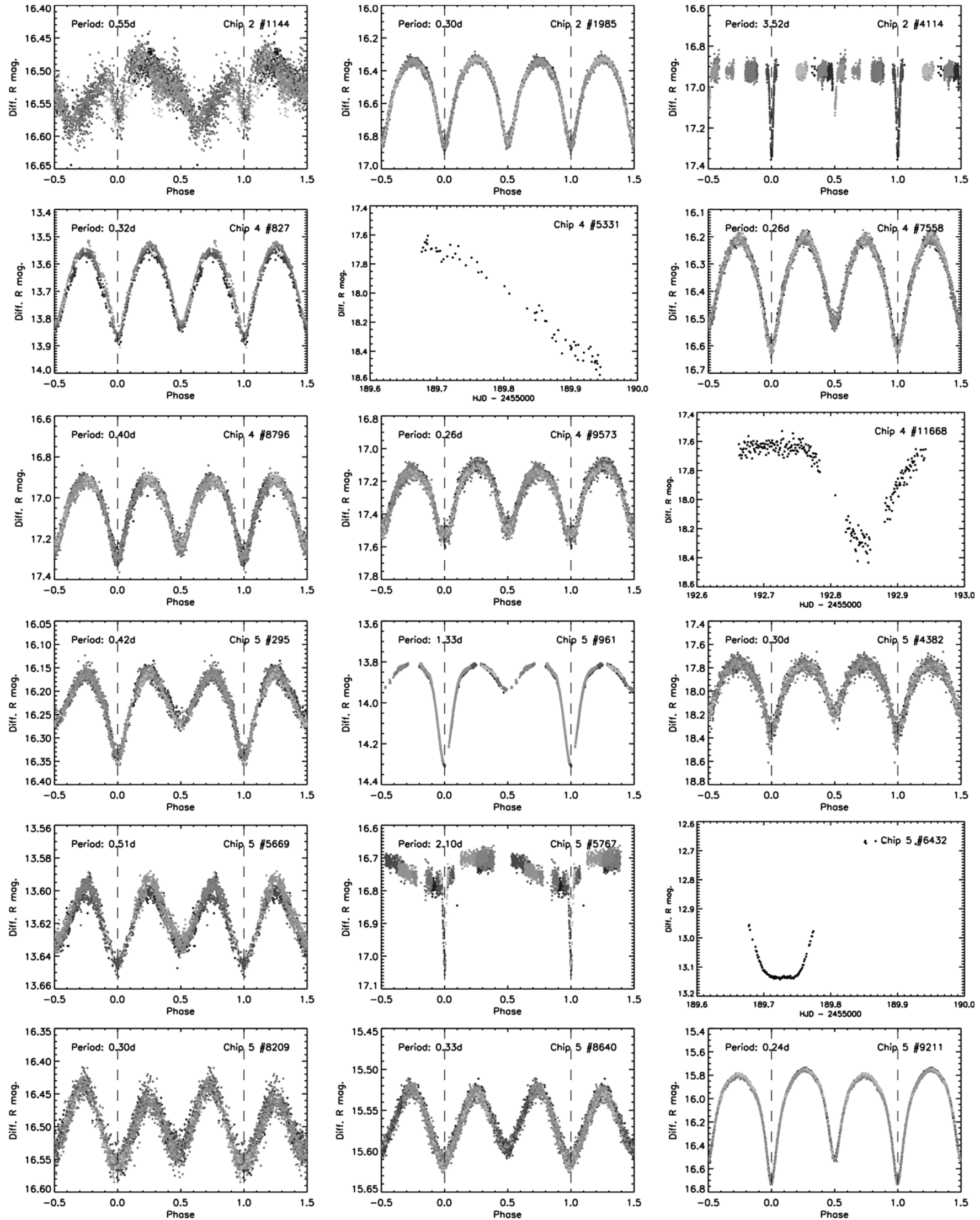

Figure 4. (Continued) 

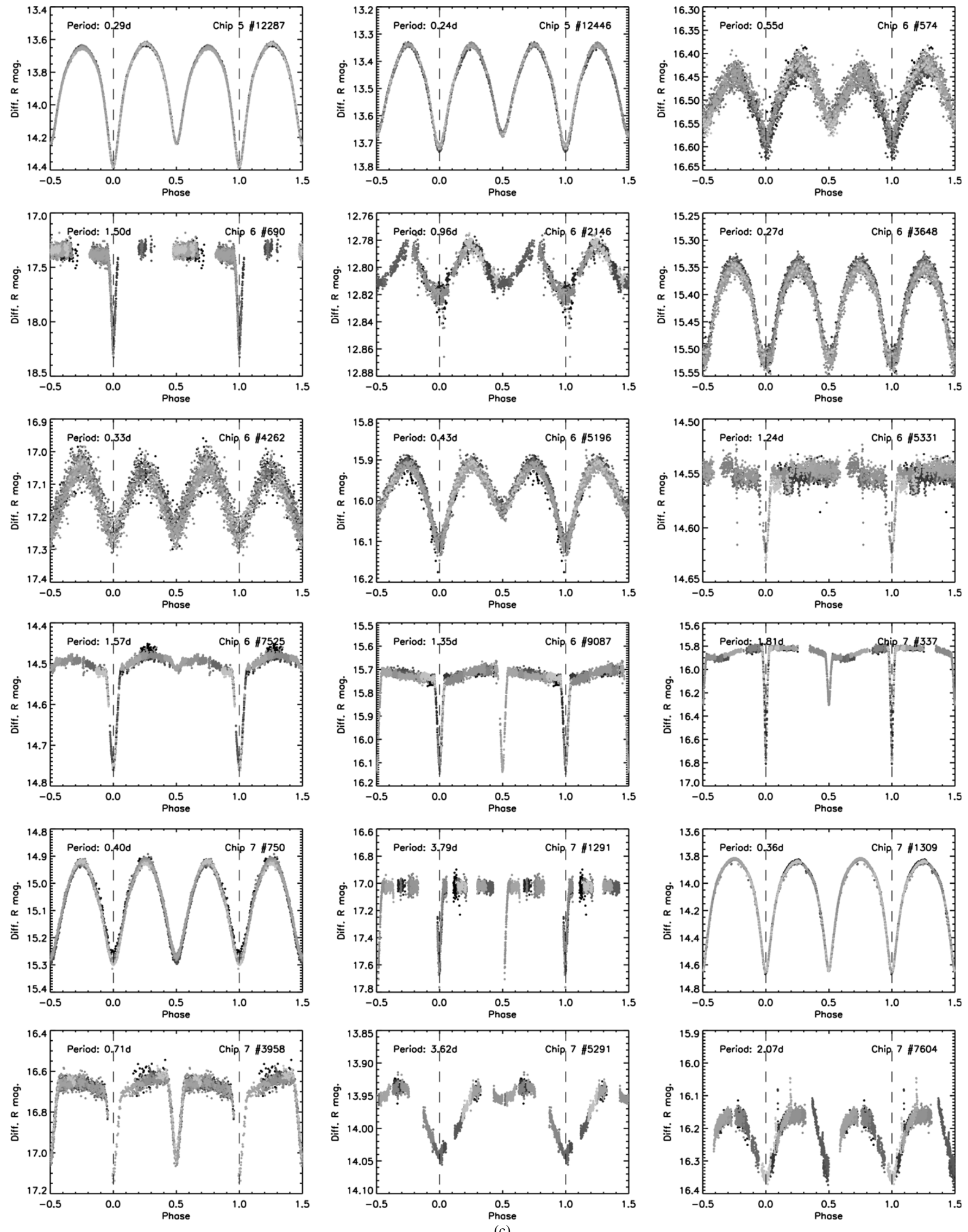

Figure 4. (Continued) 

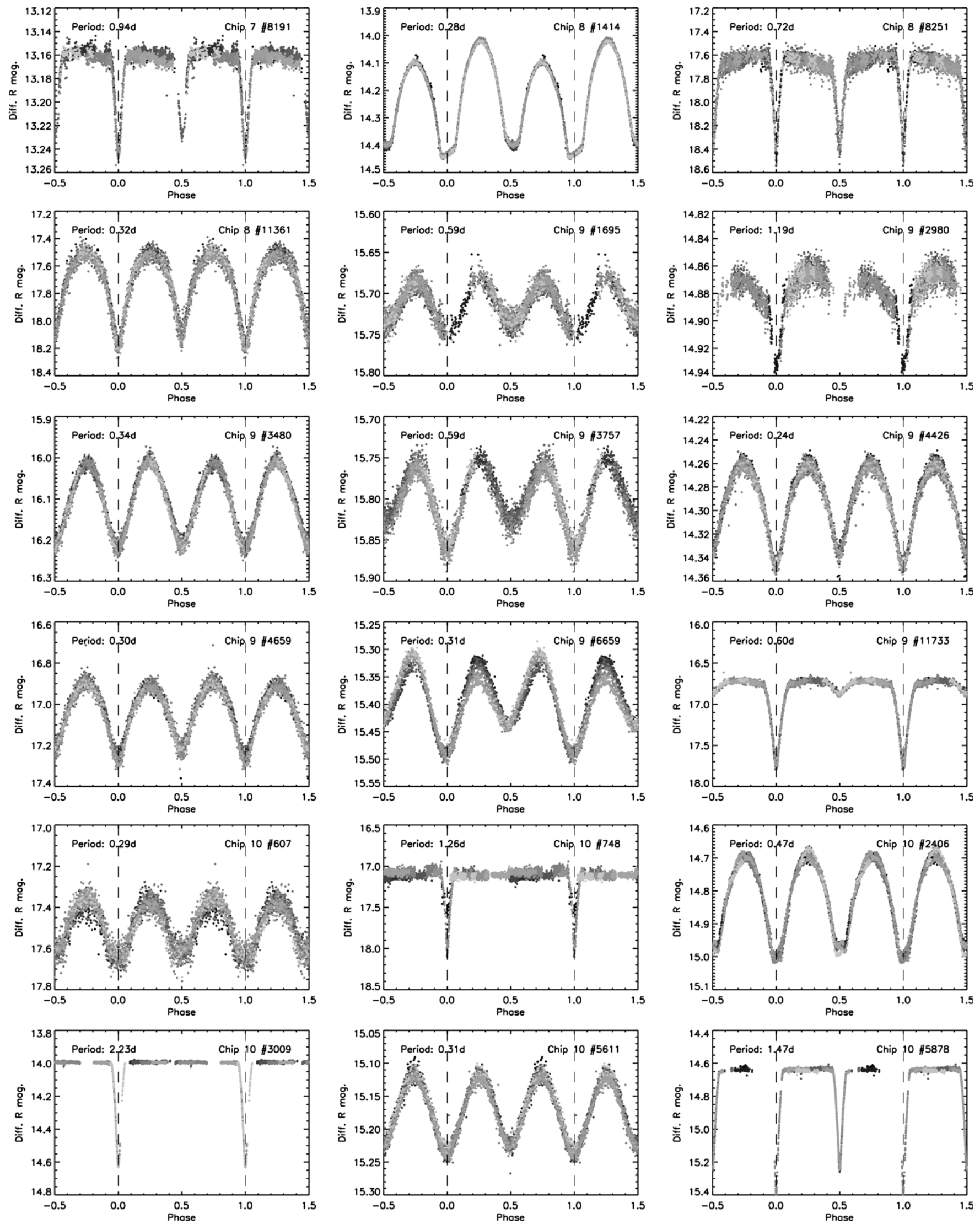

Figure 4. (Continued) 

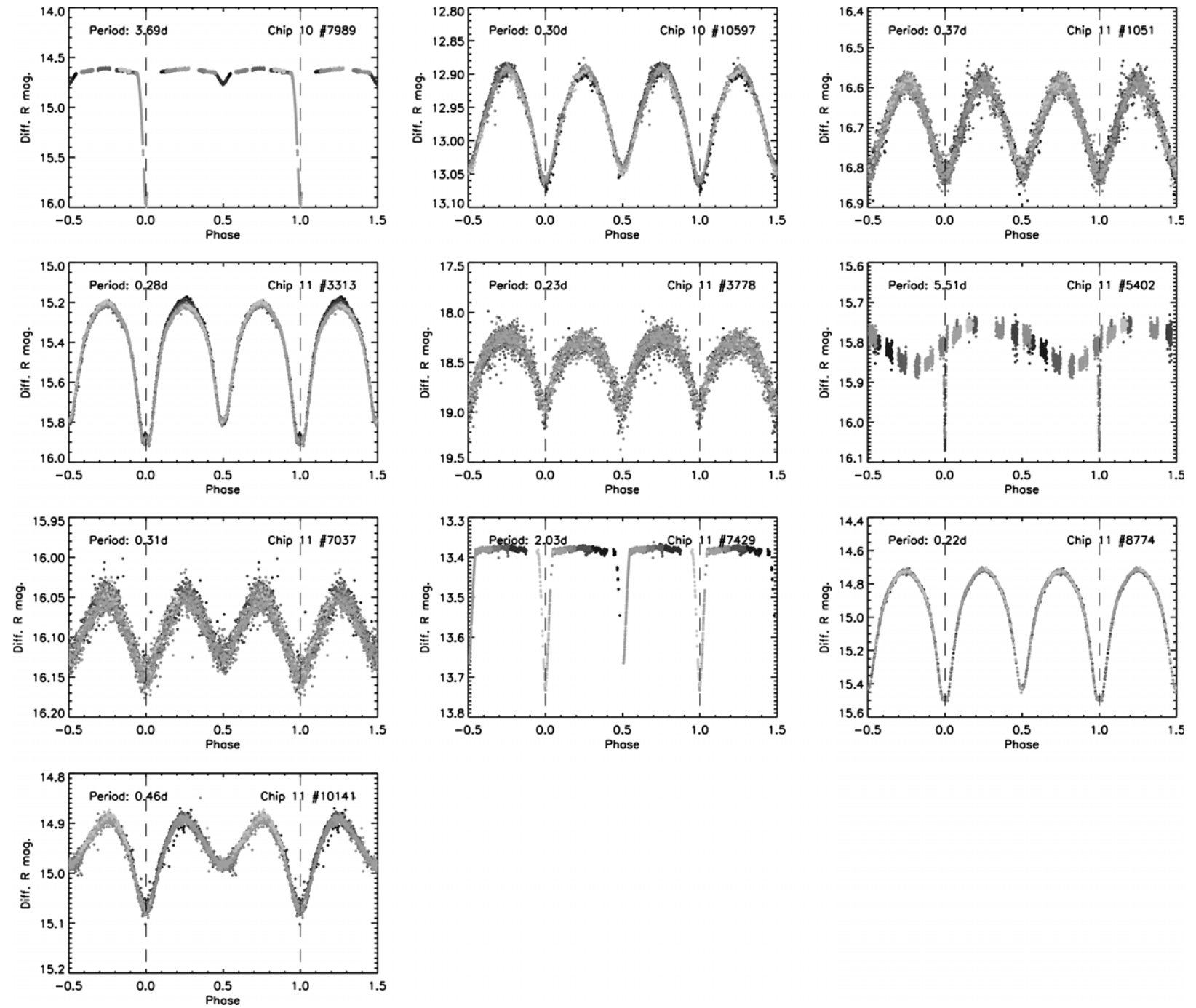

(e)

Figure 4. (Continued)

respective empirical relationships. The errors in the coefficients in the relationships are neglected, but the reported standard deviations about the relationships $(0.26 \mathrm{mag}$ and $0.49 \mathrm{mag}$ for the W UMa and detached-system calibrations, respectively) are added in quadrature to account for astrophysical effects that are not included in the relationships. In both cases, the effects of reddening are also neglected: for our assumed value of $A_{V}$ at the distance of 25 Ori, using the extinction laws of Cardelli et al. (1989) gives reddening values of $E(J-H)=0.027 \mathrm{mag}$ and $E(H-K)=0.022 \mathrm{mag}$, which in most cases are comparable to or less than the photometric measurement errors. Neglecting reddening does lead to a small systematic underestimation of the distances, generally by of order one half the measurement error in most cases for our assumed mean $A_{V}$, and always less than the full size of the error, but it is simpler than attempting to realistically estimate the reddening for each individual source.

We note that these distance relationships are likely to be inaccurate at PMS ages. Low-mass stars $\left(<1.5 M_{\odot}\right)$ younger than 10 Myr will still be on PMS tracks and can be expected to be brighter than predicted (albeit the close companions in our sample may complicate the evolutionary picture). The agreement between the list of young candidates from the color-magnitude diagram and the list from the distance estimates, however, provides some suggestion that the estimates are still meaningful in these cases (see Sections 5.2.1 and 5.2.2). Some systems also represent extrapolations beyond the formal calibrated limits of the relationships provided by Eker et al. (2009) and Bilir et al. (2008), particularly for the detached systems at apparent types later than mid-K. The distances provided in the table therefore are only intended as a guideline and should be treated with appropriate caution.

$R$ magnitudes. A single representative value for the magnitude, $R_{\text {med }}$, is given as a straight median of the measured differential $R$ magnitude for each light curve. We estimate this to be accurate on an absolute scale to $\approx 0.03-0.05 \mathrm{mag}$ for chips $0-4$ and $6-10$, and $\approx 0.07$ mag for chips 5 and 11 (see Section 4.2). The light curve amplitude variation, $\Delta R$, is calculated as the difference between the 1st and 99th percentiles of the measured $R$ magnitudes for each light curve, in order to reject possible unflagged outliers and allow for a certain amount of statistical deviation. This is similar to the range used by Basri et al. (2010). 

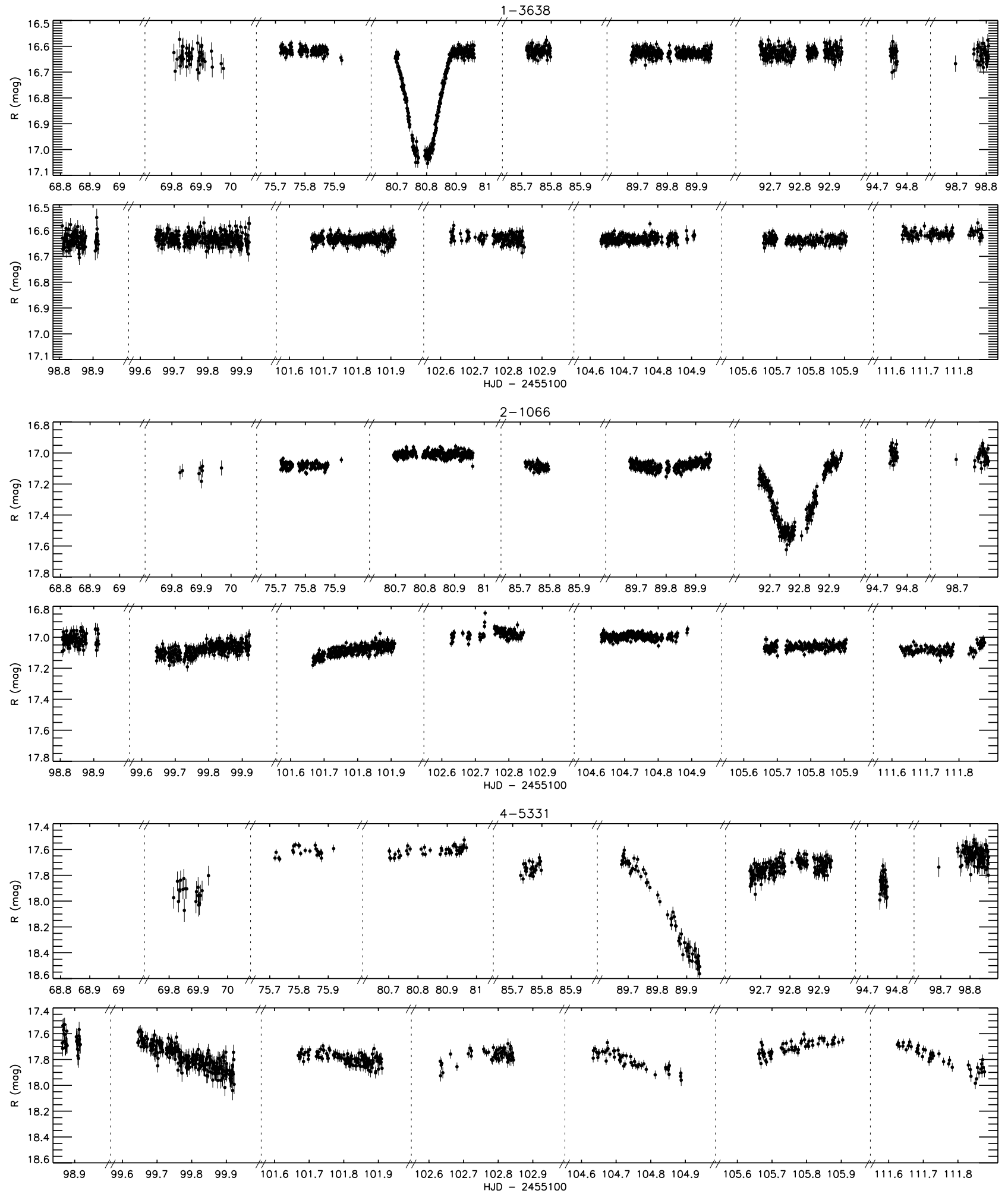

(a)

Figure 5. Full unfolded light curves for those sources for which a period could not be uniquely determined due to an insufficient number of eclipse detections or because the data did not exhibit a regular period. 5-6432 (b, bottom panel) shows sparse coverage because most of the data is saturated; the light curve drops below the saturation threshold during the eclipse, however.

\subsection{Identifying Young Binaries}

We identify young binary candidates in our sample with a color-magnitude selection, and then follow several approaches to check for consistency, detailed below. We find nine candidates that are worthy of follow-up (see beginning of Table 1).

\subsubsection{Color-Magnitude Selection}

In Figure 6, we plot the complete set of 82 binary systems in a color-magnitude diagram, using our median-measured $R$ values and the single-epoch 2MASS $K_{s}$ magnitudes (excepting those with poor $K_{s}$ measurements, i.e., those with 2MASS quality 

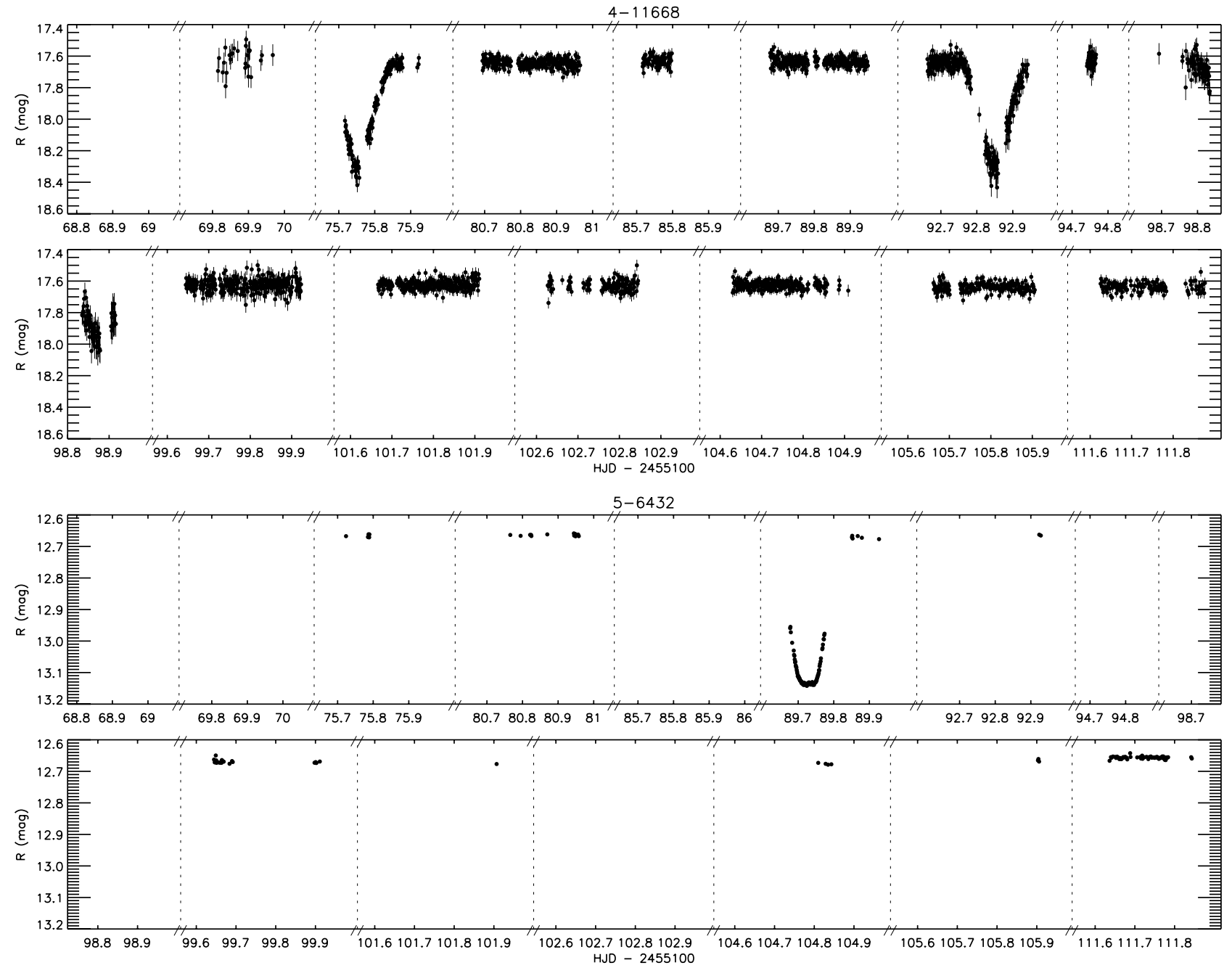

(b)

Figure 5. (Continued)

flags worse than "C" or error bars $>0.1 \mathrm{mag}$ - see Section 2.3). Isochrones are overplotted which are based on the Siess et al. (2000) models. These are tuned to fit the Pleiades main sequence at $100 \mathrm{Myr}$ (Guieu et al. 2010; Stauffer et al. 2007; Jeffries et al. 2007), and then converted to $R$ versus $R-K_{s}$ using polynomial fits to young star colors (Kenyon \& Hartmann 1995). The blue triangles mark the locations of previously identified (single) WTTS stars identified by Briceño et al. (2007) which are also found in our data and in the 2MASS catalog. They lie along the $10 \mathrm{Myr}$ isochrone, in good agreement with the $10 \mathrm{Myr}$ age determined by Briceño et al. (2007) also using Siess et al. isochrones, and supporting our placement of the isochrones in the diagram.

The uncertainties of magnitudes and colors are dominated by the amplitude of the physical photometric variability. As a result the error bars in Figure 6 are assigned to represent the overall variability of the light curves (half the peak-to-peak amplitude). In the absence of multi-epoch 2MASS measurements, we assume the variability in $K_{s}$ to be the same as that in the PTF $R$ measurements for the purposes of the error bars, and since the $R$ and $K_{s}$ are not contemporaneous, we assume the errors to be independent. We treat the WTTS sources in the same way to allow for their variability as well.
Those systems lying below the marked main sequence are taken to be unassociated with the 25 Ori/OB1a association. Those falling above the main sequence (the PMS region for the assumed 25 Ori distance and reddening) are highlighted with star symbols, and identified as candidate young systems. This criterion yields nine systems: 0-7220, 2-1066, 2-1144, 4-5331, 5-12446, 7-5291, 7-7604, 10-10597, and 11-5402 (see Table 1). For comparison, we ran a Besançon Galactic model simulation for the PTF Orion field using the default model parameters ${ }^{21}$ (Robin et al. 2003). This allows us to estimate how many field stars might be expected to lie in the PMS region. Scaling the fraction of sources found to lie above the $330 \mathrm{pc}$ main sequence in the simulation to the size of our binary sample yields $\approx 2-5$ expected field sources, depending on the exact location of the main-sequence cut.

There is an ambiguity with regard to binary systems on the color-magnitude diagram in that the mass ratios of the systems are unknown. For systems with a small mass ratio, one star dominates the luminosity, and hence the system's location on the diagram would fall as expected for a single star of the same type and color (diverging evolutionary effects due to

21 http://model.obs-besancon.fr/ 
Table 1

New Eclipsing Binaries from the PTF Orion Data

\begin{tabular}{|c|c|c|c|c|c|c|c|c|c|c|c|c|}
\hline \multirow[b]{2}{*}{ ID } & \multirow[b]{2}{*}{$\begin{array}{l}\text { R.A. } \\
\text { (deg) }\end{array}$} & \multirow[b]{2}{*}{$\begin{array}{l}\text { Decl. } \\
\text { (deg) }\end{array}$} & \multirow[b]{2}{*}{ 2MASS/USNOB1.0 } & \multicolumn{3}{|c|}{ 2MASS } & \multirow[b]{2}{*}{ Class. } & \multirow[b]{2}{*}{$\begin{array}{c}P \\
\text { (days) }\end{array}$} & \multirow[b]{2}{*}{$\begin{array}{c}T_{0} \\
\text { (HJD-2455000) }\end{array}$} & \multirow[b]{2}{*}{$\begin{array}{l}\text { Distance } \\
(\mathrm{pc})\end{array}$} & \multirow[b]{2}{*}{$\begin{array}{l}R_{\text {med }}{ }^{\mathrm{n}} \\
(\mathrm{mag})\end{array}$} & \multirow[b]{2}{*}{$\begin{array}{c}\Delta R \\
(\mathrm{mag})\end{array}$} \\
\hline & & & & $\begin{array}{c}J \\
(\mathrm{mag})\end{array}$ & $\begin{array}{c}H \\
(\mathrm{mag})\end{array}$ & $\begin{array}{c}K_{s} \\
(\mathrm{mag})\end{array}$ & & & & & & \\
\hline \multicolumn{13}{|c|}{ Pre-main-sequence low-mass binary candidates } \\
\hline $0-7220$ & 80.26648 & 2.67426 & J05210397+0240275 & $12.69 \pm 0.02$ & $12.06 \pm 0.02$ & $11.89 \pm 0.02$ & $\mathrm{D}$ & $0.679631 \pm 0.001025$ & $205.83808 \pm 0.00025$ & $349 \pm 83$ & 14.58 & 0.06 \\
\hline $2-1066^{\mathrm{a}}$ & 81.49801 & 1.96661 & J05255953+0157593 & $13.64 \pm 0.03$ & $13.04 \pm 0.03$ & $12.76 \pm 0.03$ & $\mathrm{D}$ & (1) & $192.77787 \pm 0.00050$ & $428 \pm 109$ & 17.06 & 0.58 \\
\hline 2-1144 & 81.45159 & 1.97448 & J05254838+0158276 & $13.09 \pm 0.03$ & $12.43 \pm 0.03$ & $12.20 \pm 0.03$ & $\mathrm{D}$ & $0.554431 \pm 0.001839$ & $201.70360 \pm 0.00075$ & $337 \pm 83$ & 16.53 & 0.14 \\
\hline $4-5331^{\mathrm{b}}$ & 82.40622 & 2.35695 & J05293750+0221251 & $14.36 \pm 0.04$ & $13.60 \pm 0.04$ & $13.17 \pm 0.04$ & $\mathrm{D}$ & & $189.94145 \pm 1.00000$ & $270 \pm 72$ & 17.75 & 0.87 \\
\hline $7-7604^{c}$ & 80.77483 & 1.64755 & $\mathrm{~J} 05230596+0138511$ & $13.09 \pm 0.03$ & $12.39 \pm 0.02$ & $12.18 \pm 0.03$ & $\mathrm{D}$ & $2.071092 \pm 0.009377$ & $211.69810 \pm 0.00158$ & $322 \pm 78$ & 16.18 & 0.23 \\
\hline $11-5402^{\mathrm{d}}$ & 83.18037 & 1.31577 & J05324332+0118566 & $13.44 \pm 0.03$ & $12.81 \pm 0.03$ & $12.55 \pm 0.03$ & $\mathrm{D}$ & $5.505740 \pm 0.355271$ & $199.83101 \pm 0.00032$ & $391 \pm 98$ & 15.79 & 0.29 \\
\hline \multicolumn{13}{|c|}{ Other low-mass candidates } \\
\hline $2-822$ & 81.59643 & 1.93894 & J05262316+0156196 & $15.26 \pm 0.07$ & $14.67 \pm 0.07$ & $14.29 \pm 0.08$ & $\mathrm{D}$ & $0.920431 \pm 0.000166$ & $180.83174 \pm 0.00012$ & $694 \pm 245$ & 16.82 & 0.89 \\
\hline $5-5767$ & 83.10952 & 2.38606 & J05322632+0223097 & $15.16 \pm 0.06$ & $14.58 \pm 0.06$ & $14.32 \pm 0.08$ & $\mathrm{D}$ & $2.104862 \pm 0.002494$ & $211.71483 \pm 0.00044$ & $946 \pm 326$ & 16.73 & 0.33 \\
\hline \multicolumn{13}{|c|}{ Pre-main-sequence close-binary candidates } \\
\hline $5-12446^{\circ}$ & 83.34683 & 2.97482 & J05332318+0258281 & $11.99 \pm 0.03$ & $11.47 \pm 0.02$ & $11.34 \pm 0.03$ & $\mathrm{C}$ & $0.236633 \pm 0.000017$ & $204.65544 \pm 0.00003$ & $317 \pm 41$ & 13.46 & 0.39 \\
\hline $7-5291^{\mathrm{e}}$ & 80.80171 & 1.35431 & J05231240+0121151 & $12.13 \pm 0.02$ & $11.44 \pm 0.02$ & $11.31 \pm 0.02$ & $\mathrm{C}$ & $3.623108 \pm 0.023858$ & $192.86419 \pm 0.00265$ & $1442 \pm 183$ & 13.95 & 0.11 \\
\hline 10-10597 & 82.53469 & 1.81344 & J05300832+0148489 & $11.77 \pm 0.03$ & $11.27 \pm 0.03$ & $11.13 \pm 0.02$ & $\mathrm{C}$ & $0.299531 \pm 0.000050$ & $192.72915 \pm 0.00008$ & $348 \pm 45$ & 12.96 & 0.18 \\
\hline \multicolumn{13}{|c|}{ Other binaries of interest } \\
\hline $0-3542$ & 80.00204 & 2.25436 & J05200049+0215159 & $14.27 \pm 0.03$ & $13.74 \pm 0.02$ & $13.51 \pm 0.04$ & $\mathrm{C}$ & $0.284425 \pm 0.000077$ & $199.77639 \pm 0.00023$ & $934 \pm 122$ & 15.62 & 0.28 \\
\hline $0-9653^{\circ}$ & 80.29957 & 2.93869 & J05211191+0256189 & $14.70 \pm 0.05$ & $14.18 \pm 0.04$ & $13.88 \pm 0.06$ & $\mathrm{C}$ & $0.910619 \pm 0.001313$ & $202.84000 \pm 0.10000$ & $2273 \pm 339$ & 16.23 & 0.29 \\
\hline $9-2980^{1}$ & 81.86439 & 0.98081 & J05272748+0058506 & $14.39 \pm 0.04$ & $14.12 \pm 0.04$ & $14.11 \pm 0.07$ & $\mathrm{D}$ & $1.190701 \pm 0.003662$ & $175.79416 \pm 0.00062$ & $2923 \pm 900$ & 14.87 & 0.07 \\
\hline $11-7037$ & 82.87870 & 1.50007 & J05313088+0130004 & $15.01 \pm 0.05$ & $14.50 \pm 0.07$ & $14.25 \pm 0.09$ & $\mathrm{C}$ & $0.306948 \pm 0.000228$ & $199.74559 \pm 0.00116$ & $1395 \pm 235$ & 16.09 & 0.13 \\
\hline $11-8774^{f}$ & 83.32166 & 1.68053 & J05331720+0140498 & $13.23 \pm 0.03$ & $12.62 \pm 0.03$ & $12.47 \pm 0.03$ & $\mathrm{C}$ & $0.215651 \pm 0.000007$ & $180.94621 \pm 0.00004$ & $450 \pm 60$ & 14.83 & 0.78 \\
\hline \multicolumn{13}{|c|}{ Other binaries } \\
\hline $0-1580$ & 79.94125 & 2.02664 & J05194592+0201361 & $14.35 \pm 0.04$ & $13.91 \pm 0.04$ & $13.78 \pm 0.05$ & $\mathrm{D}$ & $2.061758 \pm 0.001051$ & $198.80653 \pm 0.00012$ & $1334 \pm 376$ & 15.56 & 0.52 \\
\hline $0-4268$ & 80.46153 & 2.33903 & J05215079+0220205 & $13.03 \pm 0.03$ & $12.67 \pm 0.02$ & $12.66 \pm 0.03$ & $\mathrm{D}$ & $5.167944 \pm 0.176421$ & $202.79638 \pm 0.00003$ & $1233 \pm 307$ & 13.90 & 0.70 \\
\hline $0-4407$ & 80.17316 & 2.35519 & J05204154+0221186 & $14.60 \pm 0.03$ & $14.07 \pm 0.04$ & $13.97 \pm 0.07$ & $\mathrm{D}$ & $0.701540 \pm 0.001087$ & $189.75110 \pm 0.00039$ & $1296 \pm 389$ & 16.07 & 0.15 \\
\hline $0-5197$ & 80.45244 & 2.44664 & J05214859+0226480 & $16.20 \pm 0.13$ & $15.42 \pm 0.11$ & $15.60 \pm 0.25$ & $\mathrm{C}$ & $0.273236 \pm 0.000064$ & $198.80522 \pm 0.00047$ & $2006 \pm 627$ & 17.24 & 0.80 \\
\hline $0-5673$ & 80.06399 & 2.50293 & J05201536+0230104 & $14.91 \pm 0.05$ & $14.39 \pm 0.04$ & $14.34 \pm 0.08$ & $\mathrm{D}$ & $0.543151 \pm 0.000185$ & $175.83572 \pm 0.00037$ & $1786 \pm 602$ & 15.93 & 0.19 \\
\hline $0-8036^{\mathrm{g}}$ & 80.03662 & 2.76290 & 0927-0078105 & $\ldots$ & $\ldots$ & $\ldots$ & $\mathrm{D}$ & $0.597359 \pm 0.000604$ & $204.70458 \pm 0.00048$ & $\ldots$ & 18.09 & 0.75 \\
\hline $0-8177$ & 80.28231 & 2.77769 & J05210774+0246397 & $15.98 \pm 0.08$ & $15.82 \pm 0.14$ & $>15.21$ & $\mathrm{C}$ & $0.304414 \pm 0.000214$ & $204.74716 \pm 0.00047$ & $\ldots$ & 17.11 & 0.25 \\
\hline $0-9790$ & 80.42457 & 2.95301 & J05214195+0257109 & $15.96 \pm 0.10$ & $15.77 \pm 0.14$ & $15.49 \pm 0.23$ & $\mathrm{C}$ & $0.326311 \pm 0.000147$ & $199.72763 \pm 0.00036$ & $3842 \pm 1112$ & 17.12 & 0.43 \\
\hline $1-1223$ & 80.94578 & 2.00642 & $\mathrm{~J} 05234700+0200225$ & $15.26 \pm 0.05$ & $14.80 \pm 0.07$ & $14.87 \pm 0.13$ & $\mathrm{D}$ & $1.252181 \pm 0.000334$ & $192.80870 \pm 0.00012$ & $3488 \pm 1528$ & 15.91 & 1.59 \\
\hline $1-2659$ & 80.80171 & 2.18552 & J05231240+0211079 & $15.99 \pm 0.11$ & $15.90 \pm 0.19$ & $15.12 \pm 0.16$ & $\mathrm{D}$ & $0.960161 \pm 0.002785$ & $189.89204 \pm 0.00025$ & $1045 \pm 585$ & 16.67 & 0.59 \\
\hline $1-3638^{\mathrm{a}}$ & 81.08221 & 2.31406 & $\mathrm{~J} 05241975+0218507$ & $15.68 \pm 0.07$ & $15.46 \pm 0.12$ & $15.43 \pm 0.20$ & $\mathrm{D}$ & $\ldots$ & $180.78801 \pm 0.00028$ & $5650 \pm 3628$ & 16.63 & 0.43 \\
\hline
\end{tabular}


Table 1

(Continued)

\begin{tabular}{|c|c|c|c|c|c|c|c|c|c|c|c|c|}
\hline \multirow[b]{2}{*}{ ID } & \multirow[b]{2}{*}{$\begin{array}{l}\text { R.A. } \\
(\operatorname{deg})\end{array}$} & \multirow[b]{2}{*}{$\begin{array}{l}\text { Decl. } \\
(\mathrm{deg})\end{array}$} & \multirow[b]{2}{*}{ 2MASS/USNOB1.0 } & \multicolumn{3}{|c|}{ 2MASS } & \multirow[b]{2}{*}{ Class. } & \multirow[b]{2}{*}{$\begin{array}{c}P \\
\text { (days) } \\
\end{array}$} & \multirow[b]{2}{*}{$\begin{array}{c}T_{0} \\
\text { (HJD-2455000) }\end{array}$} & \multirow[b]{2}{*}{$\begin{array}{l}\text { Distance } \\
\text { (pc) }\end{array}$} & \multirow[b]{2}{*}{$\begin{array}{l}R_{\text {med }}{ }^{\mathrm{n}} \\
(\mathrm{mag})\end{array}$} & \multirow[b]{2}{*}{$\begin{array}{c}\Delta R \\
(\mathrm{mag})\end{array}$} \\
\hline & & & & $\begin{array}{c}J \\
(\mathrm{mag})\end{array}$ & $\begin{array}{c}H \\
(\mathrm{mag})\end{array}$ & $\begin{array}{c}K_{s} \\
(\mathrm{mag})\end{array}$ & & & & & & \\
\hline 8-11361 & 81.57440 & 1.82366 & 0918-0060816 & & & & $\mathrm{C}$ & $0.318375 \pm 0.000106$ & $180.84402 \pm 0.00037$ & & 17.65 & 0.75 \\
\hline $9-1695$ & 82.19286 & 0.85685 & $\mathrm{~J} 05284629+0051248$ & $15.15 \pm 0.04$ & $14.96 \pm 0.07$ & $14.75 \pm 0.12$ & $\mathrm{C}$ & $0.594296 \pm 0.001069$ & $175.76614 \pm 0.00116$ & $4098 \pm 747$ & 15.71 & 0.08 \\
\hline $9-3480$ & 81.96793 & 1.02633 & J05275232+0101345 & $15.14 \pm 0.05$ & $14.83 \pm 0.08$ & $14.88 \pm 0.14$ & $\mathrm{C}$ & $0.342832 \pm 0.000113$ & $204.68358 \pm 0.00041$ & $2921 \pm 557$ & 16.11 & 0.23 \\
\hline $9-3757^{\mathrm{i}}$ & 82.15440 & 1.05077 & J05283707+0103024 & $15.11 \pm 0.05$ & $14.83 \pm 0.07$ & $14.74 \pm 0.11$ & $\mathrm{C}$ & $0.585259 \pm 0.000765$ & $201.80116 \pm 0.00075$ & $3775 \pm 655$ & 15.80 & 0.13 \\
\hline $9-4426$ & 81.81013 & 1.11631 & J05271446+0106585 & $13.22 \pm 0.03$ & $12.82 \pm 0.02$ & $12.69 \pm 0.03$ & $\mathrm{C}$ & $0.239152 \pm 0.000056$ & $201.86540 \pm 0.00024$ & $698 \pm 91$ & 14.28 & 0.10 \\
\hline $9-4659$ & 81.86969 & 1.14085 & J05272875+0108267 & $15.92 \pm 0.09$ & $15.50 \pm 0.13$ & $15.49 \pm 0.22$ & $\mathrm{C}$ & $0.297846 \pm 0.000110$ & $180.84125 \pm 0.00034$ & $3024 \pm 833$ & 17.00 & 0.42 \\
\hline $9-6659$ & 82.18806 & 1.33594 & J05284514+0120089 & $14.33 \pm 0.03$ & $13.93 \pm 0.05$ & $13.86 \pm 0.05$ & $\mathrm{C}$ & $0.310999 \pm 0.000134$ & $204.68321 \pm 0.00031$ & $1458 \pm 207$ & 15.40 & 0.19 \\
\hline $9-11733$ & 82.00983 & 1.80036 & J05280235+0148015 & $15.93 \pm 0.10$ & $15.80 \pm 0.18$ & $>16.30$ & $\mathrm{D}$ & $0.602753 \pm 0.000100$ & $192.72042 \pm 0.00007$ & $\ldots$ & 16.75 & 1.06 \\
\hline $10-607$ & 82.46420 & 0.77599 & J05295140+0046337 & $16.29 \pm 0.14$ & $16.12 \pm 0.19$ & $>15.84$ & $\mathrm{C}$ & $0.289616 \pm 0.000200$ & $189.72307 \pm 0.00075$ & & 17.48 & 0.38 \\
\hline $10-748^{j}$ & 82.52387 & 0.79020 & J05300573+0047249 & $14.87 \pm 0.04$ & $14.24 \pm 0.04$ & $14.10 \pm 0.07$ & $\mathrm{D}$ & $1.261511 \pm 0.001564$ & $192.67356 \pm 0.00200$ & $1051 \pm 337$ & 17.11 & 0.92 \\
\hline $10-2406$ & 82.78685 & 0.96683 & J05310883+0058008 & $13.52 \pm 0.03$ & $13.15 \pm 0.03$ & $13.03 \pm 0.04$ & $\mathrm{C}$ & $0.468237 \pm 0.000143$ & $199.83276 \pm 0.00016$ & $1313 \pm 178$ & 14.77 & 0.33 \\
\hline $10-3009^{j}$ & 82.49377 & 1.03404 & J05295852+0102022 & $13.13 \pm 0.03$ & $12.89 \pm 0.03$ & $12.83 \pm 0.03$ & $\mathrm{D}$ & $2.234000 \pm 0.006000$ & $202.82948 \pm 0.00011$ & $1504 \pm 370$ & 13.99 & 0.63 \\
\hline $10-5611$ & 82.57626 & 1.31356 & J05301832+0118484 & $14.23 \pm 0.04$ & $13.89 \pm 0.04$ & $13.77 \pm 0.06$ & $\mathrm{C}$ & $0.308967 \pm 0.000117$ & $180.87753 \pm 0.00034$ & $1484 \pm 211$ & 15.17 & 0.14 \\
\hline $10-5878$ & 82.81800 & 1.34175 & J05311632+0120299 & $13.63 \pm 0.03$ & $13.26 \pm 0.04$ & $13.17 \pm 0.04$ & $\mathrm{D}$ & $1.466659 \pm 0.000361$ & $199.66223 \pm 0.00012$ & $1294 \pm 339$ & 14.64 & 0.64 \\
\hline $10-7989$ & 82.71804 & 1.55714 & $\mathrm{~J} 05305236+0133253$ & $14.03 \pm 0.04$ & $13.78 \pm 0.04$ & $13.61 \pm 0.06$ & $\mathrm{D}$ & $3.690487 \pm 0.008470$ & $204.88043 \pm 0.00031$ & $1611 \pm 458$ & 14.64 & 1.26 \\
\hline $11-1051^{\mathrm{j}}$ & 82.94454 & 0.82398 & J05314668+0049260 & $15.91 \pm 0.10$ & $15.62 \pm 0.12$ & $15.57 \pm 0.23$ & $\mathrm{C}$ & $0.372703 \pm 0.000221$ & $201.79040 \pm 0.00050$ & $4185 \pm 1216$ & 16.68 & 0.27 \\
\hline $11-3313$ & 83.03217 & 1.07005 & J05320774+0104117 & $14.06 \pm 0.03$ & $13.62 \pm 0.03$ & $13.55 \pm 0.04$ & $\mathrm{C}$ & $0.281535 \pm 0.000019$ & $180.87347 \pm 0.00006$ & $1128 \pm 152$ & 15.33 & 0.70 \\
\hline $11-3778$ & 83.06084 & 1.12772 & 0911-0062767 & & & 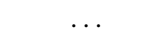 & $\mathrm{C}$ & $0.227754 \pm 0.000108$ & $180.90388 \pm 0.00060$ & & 18.40 & 0.91 \\
\hline $11-7429$ & 83.03414 & 1.54127 & J05320820+0132287 & $12.56 \pm 0.03$ & $12.30 \pm 0.03$ & $12.21 \pm 0.03$ & $\mathrm{D}$ & $2.034245 \pm 0.002301$ & $211.73886 \pm 0.00010$ & $1014 \pm 252$ & 13.38 & 0.32 \\
\hline $11-10141$ & 83.42733 & 1.82439 & $\mathrm{~J} 05334253+0149276$ & $14.25 \pm 0.04$ & $13.96 \pm 0.03$ & $13.96 \pm 0.06$ & $\mathrm{C}$ & $0.456071 \pm 0.000184$ & $201.79630 \pm 0.00022$ & $2319 \pm 327$ & 14.95 & 0.19 \\
\hline
\end{tabular}

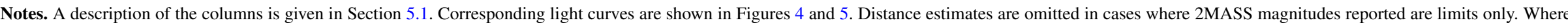

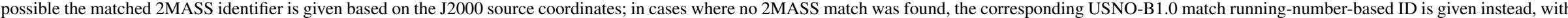

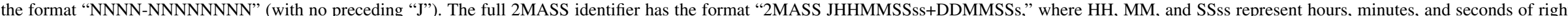

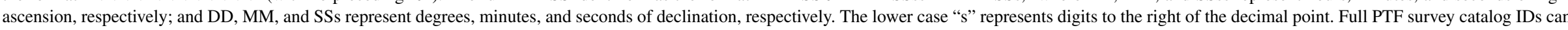

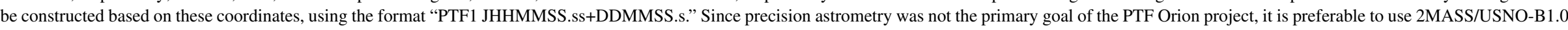
coordinates for this purpose rather than our PTF Orion measured coordinates.

${ }^{a}$ Only one eclipse obtained-period indeterminate.

${ }^{\mathrm{b}}$ Partial coverage of only one (presumed) eclipse obtained—period indeterminate, $T_{0}$ not well constrained.

${ }^{\mathrm{c}} T_{0}$ not well determined due to incomplete coverage of eclipse. Measurement error may be unreliable.

d Only one (very good) eclipse observed; period based on out-of-eclipse variation.

${ }^{\mathrm{e}}$ Unusual light curve, possible semi-detached system. Listed distance is assuming a contact binary, but using a detached-system distance estimate yields a distance of $268 \pm 64 \mathrm{pc}-$ see Section 5.4 .3 .

${ }^{\mathrm{f}}$ Very short period W UMa system-see Section 5.4.4.

g Used center of secondary eclipse for $T_{0}$, owing to poor coverage of primary

${ }^{\mathrm{h}}$ Three clear eclipses, but unable to find coherent period-possible triple system?

${ }^{\mathrm{i}}$ Nearby second source in USNO-B; chance of slight contamination.

${ }^{j}$ Secondary eclipse not evident, or primary and secondary eclipses indistinguishable—possible factor of two ambiguity in $P$.

${ }^{\mathrm{k}}$ Only two eclipses obtained-period ambiguous.

${ }^{1}$ Apparent pulsating binary-short-period oscillations seen, with $\sim 1 / 2 \mathrm{hr}$ period. See Section 5.4.4.

${ }^{\mathrm{m}}$ Somewhat distorted light curve shape-may actually represent stellar pulsations rather than a binary system (D. Bradstreet 2010, private communication).

${ }^{\mathrm{n}}$ See Section 4.2 for a discussion of zero-point accuracy.

${ }^{\circ}$ Identified as a candidate variable star by Kraus et al. (2007). 


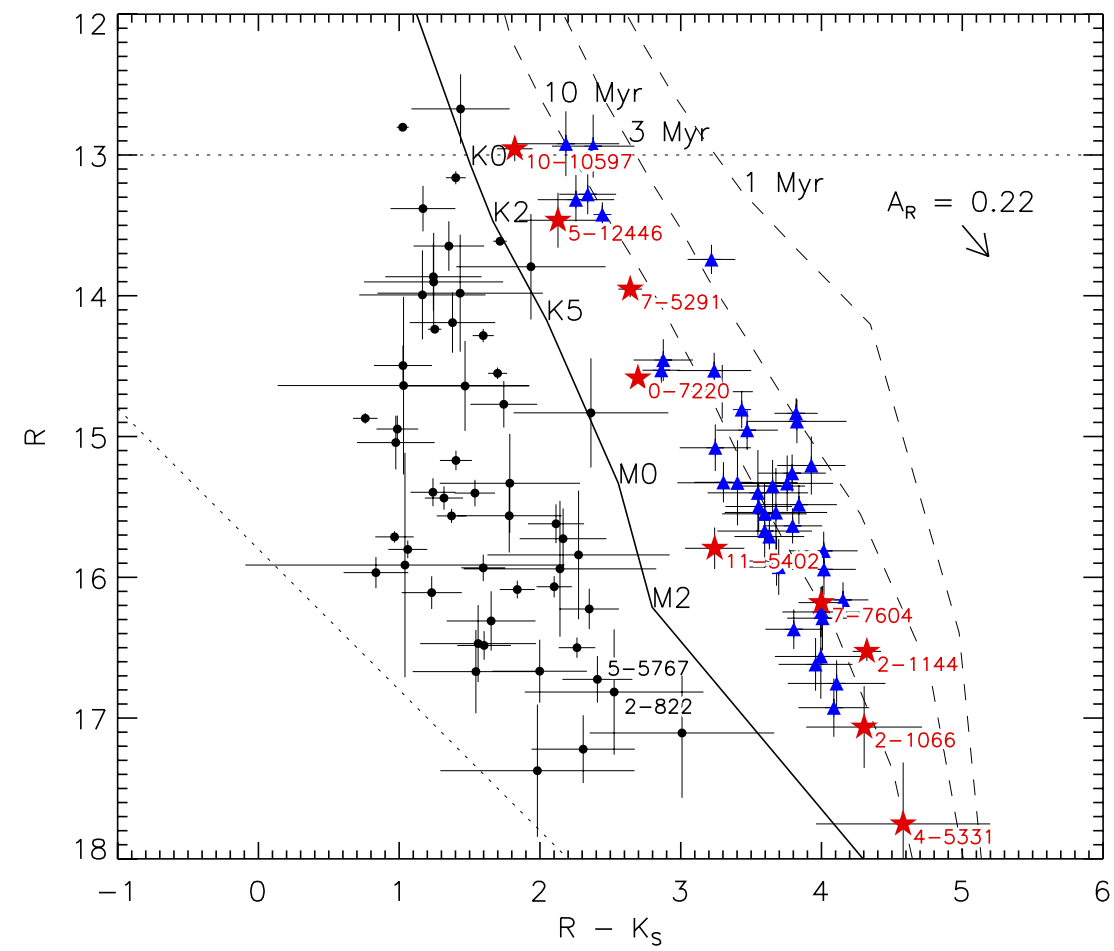

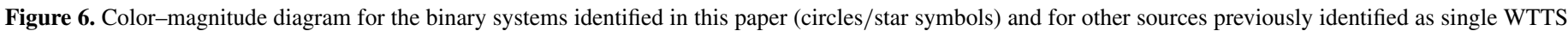

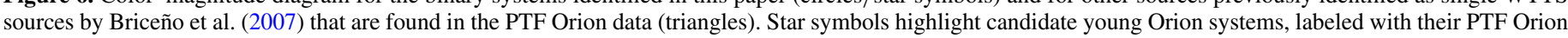

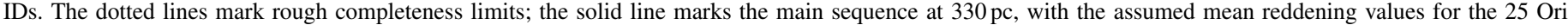

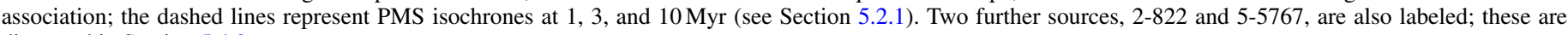
discussed in Section 5.4.2.

(A color version of this figure is available in the online journal.)

the binarity notwithstanding). However, for mass ratios near unity, the two stars contribute similar fluxes, appearing up to $\approx 0.75$ mag brighter in total than a single star of the same color. There is a possibility, therefore, that some binaries could be shifted systematically upward in the diagram compared to single stars, and that location near the OB1a/25 Ori group sequence could simply be a result of field binaries suffering from this effect. These nine systems should therefore only be considered a list of good candidate young systems from the sample, for which further spectroscopic follow-up is needed for proper confirmation. At the same time, we note that shifting downward by 0.75 mag any of those candidates on the low edge of the cluster sequence in the diagram (assuming that $R-K$ remains the same) still places them near the main sequence for the distance of the association, and does not rule out Orion membership. It is possible, for example, that binarity has affected their evolution compared to that of single stars, particularly given the close separations implied by the relatively short orbital periods of our sample. In the following sections, we investigate other consistency tests for the proposed young ages of the candidates.

\subsubsection{Distances}

Figure 7 shows a histogram of the log of the derived distances for the binaries in Table 1 (excluding those with 2MASS quality flags worse than "C" - see Section 2.3). The number of binaries per logarithmic bin increases with distance, peaking at around $\log ($ distance $/ \mathrm{pc})=3.1$ and then trailing off. This we attribute to the background field distribution of binaries in the galaxy in combination with the sensitivity limit of the sample and the logarithmic bin sizes. In addition we see a distinct peak at around $330 \mathrm{pc}$, the distance to the OB1a and 25 Ori associations adopted by Briceño et al. $(2005,2007)$. We take systems with derived distances falling at the 25 Ori/OB1a distance within a factor of 1.5 times their respective errors, and with errors below $150 \mathrm{pc}$, to be good candidates for membership in the association. This criterion yields exactly the same list of candidates as the color-magnitude diagram selection, consistent with their proposed young nature, with the exception only of source 7-5291. This source presents some peculiarities, however, and there are indications that the initial distance estimate is probably incorrect. An estimate based on the detached-system calibration being likely more appropriate than the presumed W UMa distance calibration and satisfies the above criteria. We therefore do not rule it out as a young candidate (see Section 5.4.3 for more discussion).

The same mass ratio ambiguity as discussed in the previous section can also affect the distance estimates, although probably to a lesser extent since the distance calibrations used are based on broad samples of binary systems, and therefore should represent the relation for a "typical" mass ratio. Bilir et al. (2008) report a standard deviation of 0.49 mag about the relation for detached systems, pointing out that this could be largely explained by the mass ratio ambiguity, which should lead on average to an error less than 0.4 mag; Eker et al. (2009) report a standard deviation of 0.26 mag for $\mathrm{W}$ UMa systems. In the worst possible case where a mass ratio of unity leads to a $\approx 0.75 \mathrm{mag}$ underestimate of the distance modulus, this would cause a distance underestimate of $\approx 40 \%$. Even at this extreme, however, an estimated distance at the $330 \mathrm{pc}$ mark still places the true value near the more distant OB1b association $(\approx 440$ pc; Briceño et al. 2005; Brown 


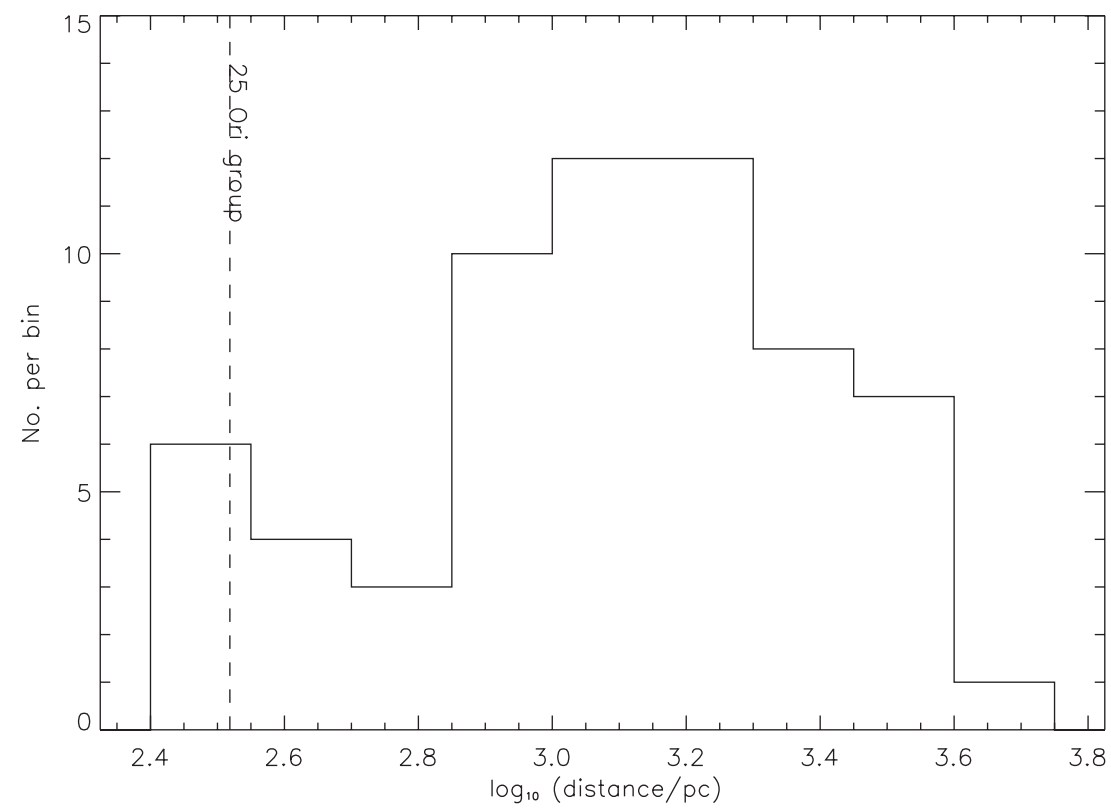

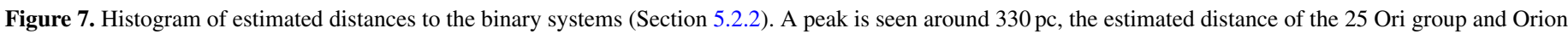
OB 1a $\left(\log _{10}\right.$ distance $=2.52$, marked with a dashed line $)$. Bin size is $0.15 \log _{10}($ distance $/ \mathrm{pc})$.

et al. 1999), and such a source would remain of potential interest.

\subsubsection{Stellar Variability}

Intrinsic stellar variability can also be a useful indicator for young stars, which tend to be more spotted and active (e.g., Briceño et al. 2001; Lamm et al. 2004). In Figure 8, we plot an estimate of the intrinsic variability of the detached binary systems in our sample for which a meaningful estimate could be calculated. All "close" systems are omitted, as is 5-961, a likely semi-detached system, since they show continuous strong ellipsoidal variation which confuses the intrinsic variability measurement. To quantify the intrinsic stellar variability, the out-of-eclipse rms $R$ magnitude is first calculated as the outlierresistant standard deviation for a given light curve between orbital phases $0.1-0.4$ and $0.6-0.9$, effectively cutting out the eclipses in the detached binaries (assuming circular orbits, which is reasonable given the short orbital periods in our sample). To account for the apparent noise floor in Figure 3 a constant $0.004 \mathrm{mag}$ is added in quadrature to the measurement errors. Variability is then calculated by subtracting the median measurement error for each light curve (in quadrature) in order to leave only intrinsic variability. This quantity provides an estimate of the stellar activity, though with the caveat that lowlevel ellipsoidal variations may also be included in the metric. The value of the reduced $\chi^{2}$ compared to a constant $R$ magnitude at the weighted mean for each source is also plotted, to provide an indication of the statistical significance of the measure.

The effects of photon error having been removed from the variability diagram, the general increasing trend with fainter magnitudes is attributed to the tendency toward increased activity at later spectral types, since more of these are seen at the faint end of the sample. The same candidate young binary systems (Section 5.2.1) are again marked with star symbols (excluding the three "close" systems, 7-5291, 5-12446, and 1010597). They are intrinsically more variable on average than the other detached systems in the sample, consistent with their suspected young ages. (The elevated source below 11-5402 is an additional system that shows marked variation probably caused by hot or cold spots (7-337—see Figure 4(c)).)

\subsubsection{Proper Motions}

Proper motions provide a further consistency check on the candidate young binary systems, although for the 25 Ori cluster and the broader Orion region, the proper motion is near zero, making it hard to distinguish between 25 Ori association members, Ori OB1 members, and more distant background sources (see de Zeeuw et al. 1999; Kharchenko et al. 2005). Figure 9 shows the proper motions of each of the binary systems, taken from the recently released PPMXL catalog of positions and proper motions (Roeser et al. 2010). Kharchenko et al. (2005) reported a proper motion for the 25 Ori cluster (identified as ASCC 16 in their catalog) of $0.75 \pm 0.22 \mathrm{mas} \mathrm{yr}^{-1}$ in right ascension and $-0.18 \pm 0.29$ mas yr$^{-1}$ in declination based on the brighter stars in the cluster. Briceño et al. (2007) report a $\pm 1.7 \mathrm{~km} \mathrm{~s}^{-1}$ width to the core of the peak of their distribution of radial velocity (RV) measurements for the cluster, which corresponds to $\mathrm{a} \pm 1.09$ mas $\mathrm{yr}^{-1}$ dispersion in proper motions at $330 \mathrm{pc}$ (indicated in the figure), assuming a three-dimensionally isotropic distribution of velocities.

The nine young candidate systems are again highlighted with red star symbols. Their proper motions are largely consistent with 25 Ori/OB 1 a membership, with the exception of 5-12446, with a proper motion of $30.0 \pm 3.8 \mathrm{mas} \mathrm{yr}^{-1}$ in right ascension despite its estimated distance of $317 \pm 41 \mathrm{pc}$, which is extremely close to that of the 25 Ori/OB1a association. This suggests it is probably an interloper rather than a true member (see Section 5.4.3).

\subsubsection{Infrared Analysis}

Finally, we also searched for any existing Spitzer IRAC/ MIPS data, to check for any infrared excess that might be indicative of T-Tauri-like disks in the nine candidate young systems. Data were found for 2-1066, 2-1144, 7-5291, 7-7604, and 10-10597. These are listed in Table 2, and SEDs are shown in Figure 10, where the gray lines represent an extrapolation of 


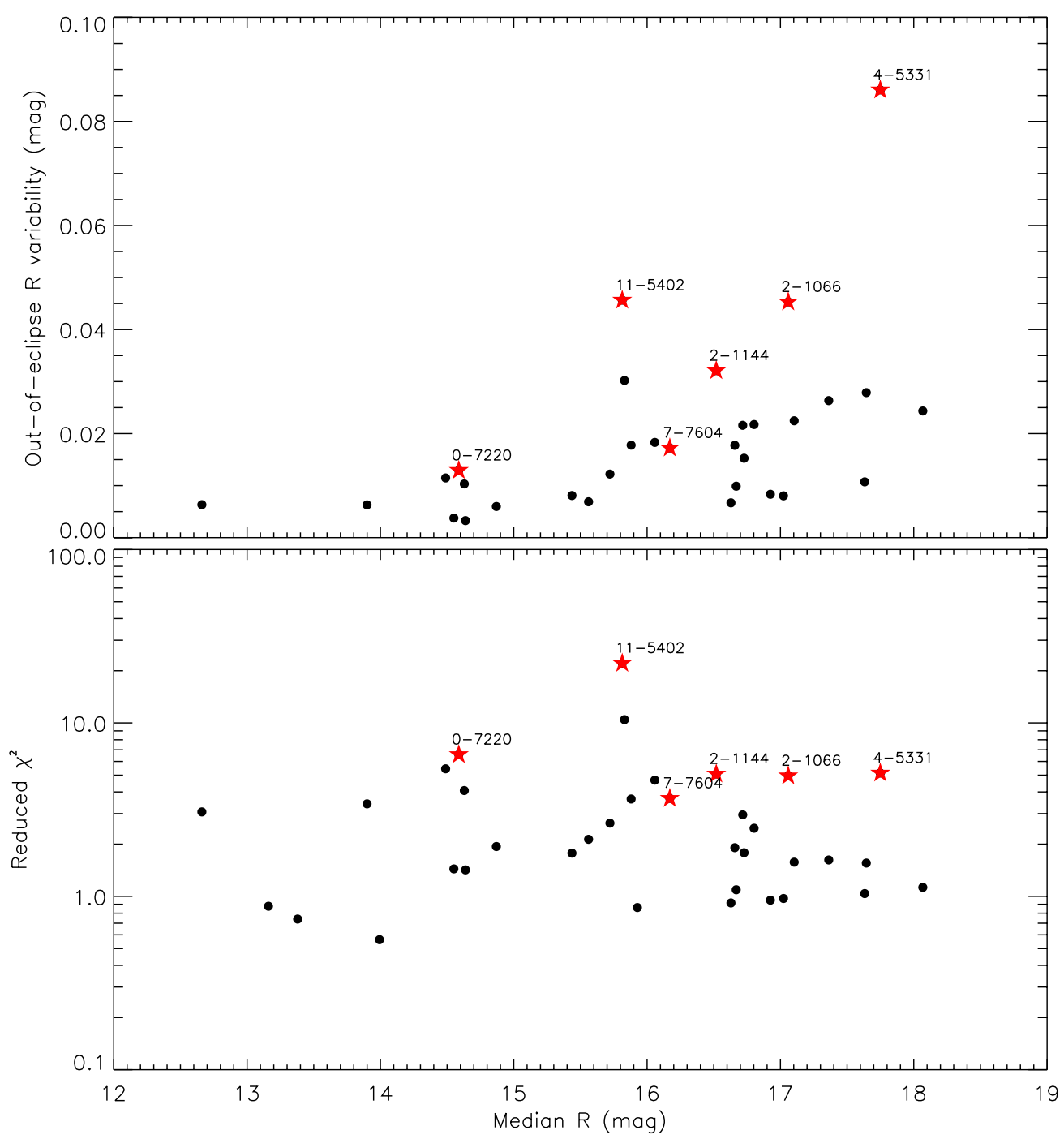

Figure 8. Intrinsic stellar variability estimates for all the binary systems classified as detached (Section 5.2.3). Star symbols highlight those that are the candidate young systems selected from the color-magnitude diagram (Figure 6). Variability (top panel) is calculated as the quadrature difference between the out-of-eclipse rms and the median measurement error for each light curve (with an additional estimated 0.004 mag noise floor added in quadrature); sources where the rms is below the median measurement error are omitted. The reduced $\chi^{2}$ against the weighted mean $R$ magnitude is shown for the same data (bottom panel), including those sources with rms smaller than the measurement error. System 5-961 is omitted from both panels since it shows significant ellipsoidal variation (Figure 4(b)).

(A color version of this figure is available in the online journal.)

Table 2

Existing Spitzer Data for Candidate Young Binaries

\begin{tabular}{lcccccccrrr}
\hline \hline ID & $J$ & $H$ & $K_{s}$ & {$[3.6]$} & {$[4.5]$} & {$[5.8]$} & {$[8]$} & {$[24]$} & $K_{s}-[24]$ & {$[3.6]-[24]$} \\
\hline $2-1066$ & $13.64 \pm 0.03$ & $13.04 \pm 0.03$ & $12.76 \pm 0.03$ & $\ldots$ & $11.84 \pm 0.06$ & $\ldots$ & $11.24 \pm 0.06$ & $8.01 \pm 0.05$ & $4.75 \pm 0.06$ & $\ldots$ \\
$2-1144$ & $13.09 \pm 0.03$ & $12.43 \pm 0.03$ & $12.20 \pm 0.03$ & $\ldots$ & $11.82 \pm 0.06$ & $\ldots$ & $11.77 \pm 0.06$ & $11.68 \pm 0.34$ & $0.52 \pm 0.34$ & $\ldots$ \\
$7-5291$ & $12.13 \pm 0.02$ & $11.44 \pm 0.02$ & $11.31 \pm 0.02$ & $11.20 \pm 0.06$ & $\ldots$ & $11.21 \pm 0.06$ & $\ldots$ & $10.47 \pm 0.12$ & $0.84 \pm 0.12$ & $0.73 \pm 0.13$ \\
$7-7604$ & $13.09 \pm 0.03$ & $12.39 \pm 0.02$ & $12.18 \pm 0.03$ & $11.97 \pm 0.06$ & $11.87 \pm 0.06$ & $11.87 \pm 0.06$ & $11.89 \pm 0.06$ & $10.18 \pm 0.11$ & $2.00 \pm 0.11$ & $1.79 \pm 0.13$ \\
$10-10597$ & $11.77 \pm 0.03$ & $11.27 \pm 0.03$ & $11.14 \pm 0.02$ & $10.93 \pm 0.06$ & $10.94 \pm 0.06$ & $10.92 \pm 0.06$ & $10.92 \pm 0.06$ & $11.48 \pm 0.20$ & $-0.34 \pm 0.20$ & $-0.55 \pm 0.21$ \\
\hline
\end{tabular}

Notes. All candidate young binaries are listed for which Spitzer IRAC/MIPS data could be found (Section 5.2.5). 2MASS $J, H$, and $K_{s}$ measurements are repeated from Table 1 for comparison. All values are in magnitudes.

the Rayleigh-Jeans slope from the $K_{s}$ measurements to guide the eye with regard to the expected photospheric flux. A color difference between any two points on such a gradient will have a value of zero, and anything lying above the slope is suggestive of an IR excess. As discussed in Section 2.3, comparing to $K_{s}$ is not optimal, but [3.6] is in many cases not available. 2-1066 seems to show a clear excess, with $K_{s}-[24]=4.75 \pm 0.06 \mathrm{mag}$. Though it lacks a [3.6] measurement, the two IRAC bands which are measured ([4.5] and [8]) also lie above the extrapolated photosphere as can be seen in the figure, supporting this conclusion (though it is also possible that the excess may be caused by confusion from the secondary in the system). The remainder show no detected excess, or at most, the possibility of a marginal very weak excess. These results are not surprising: by design, the age of the region in the field is such that for the most part the disks have largely dissipated. 


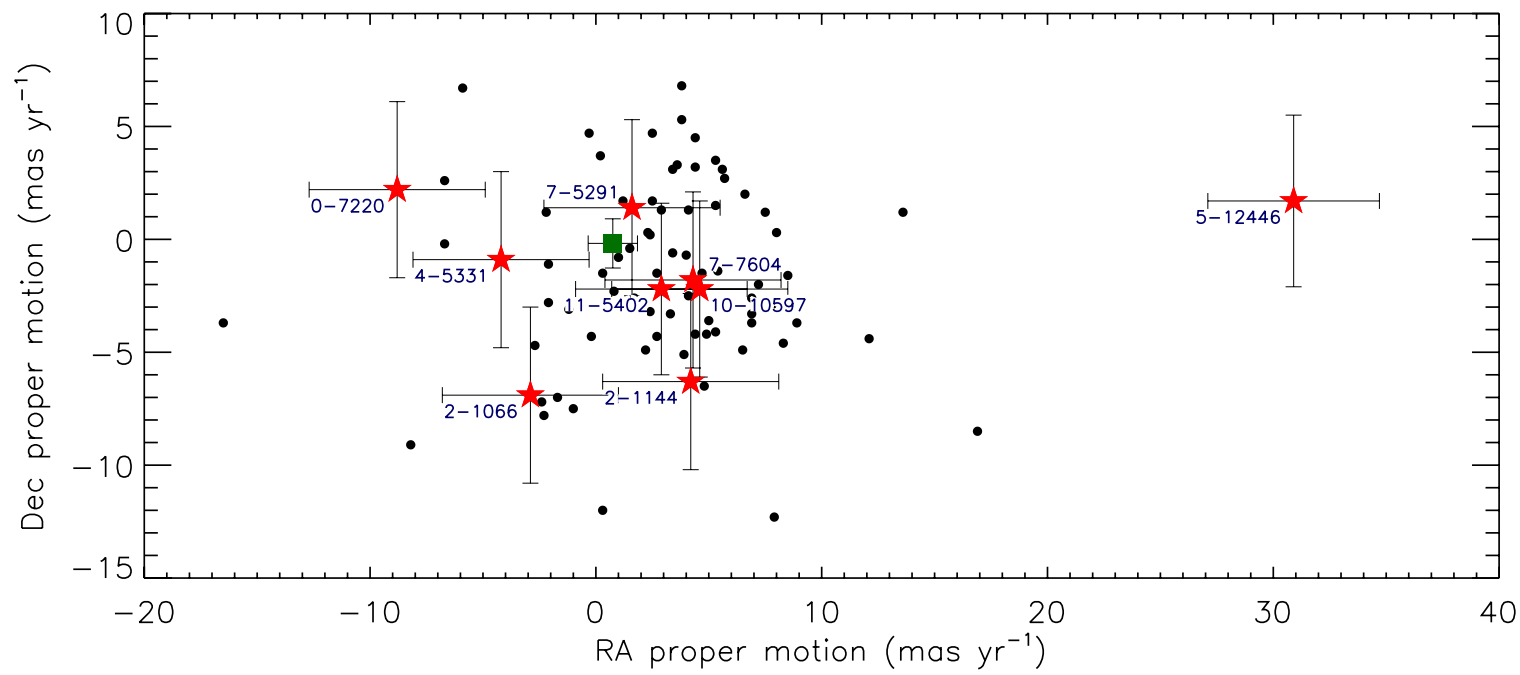

Figure 9. PPMXL proper motions for all the identified eclipsing binary star systems. Candidate young systems are marked with star symbols and have error bars indicated. Error bars for all other systems are omitted for clarity, but are similar in size. The square symbol marks the proper motion of the 25 Ori cluster measured by Kharchenko et al. (2005), with error bars representing the dispersion derived from RV measurements by Briceño et al. (2007). (See Section 5.2.4.)

(A color version of this figure is available in the online journal.)

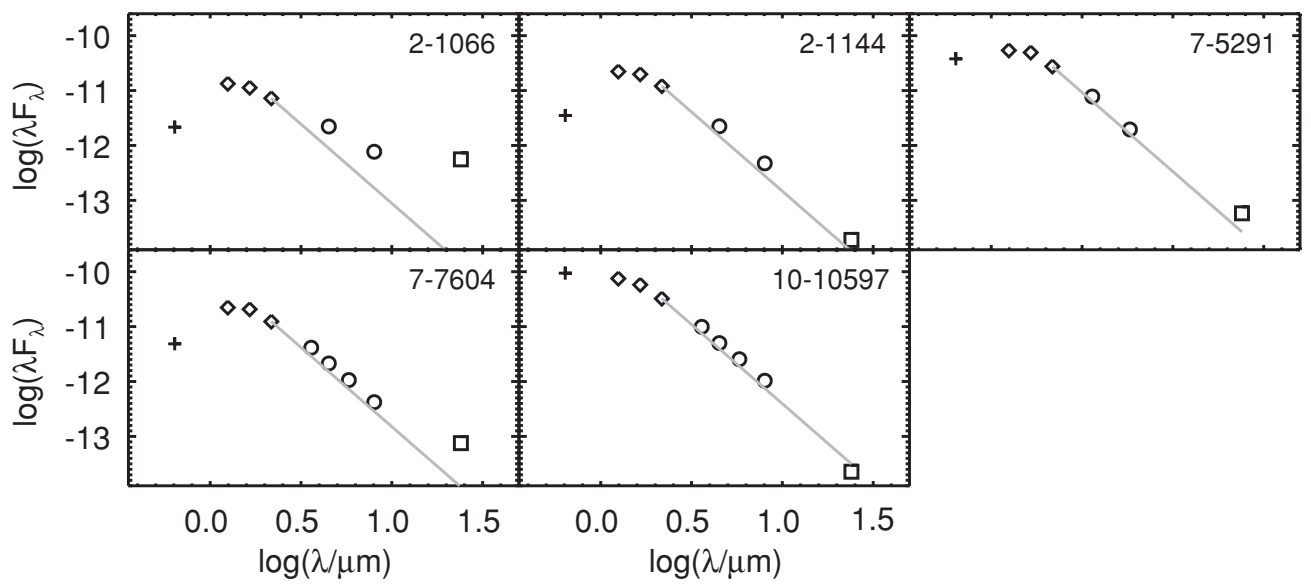

Figure 10. SEDs for the candidate young binary systems for which IR Spitzer data could be found (Section 5.2 .5 and Table 2). The SEDs are all in log $\lambda F_{\lambda}$ in cgs units ( $\left.\mathrm{erg} \mathrm{s}^{-1} \mathrm{~cm}^{-2}\right)$, against $\log \lambda$ in microns. Crosses are median PTF $R$, diamonds are 2MASS $\left(J H K_{s}, 1.25,1.65\right.$, and $\left.2.17 \mu \mathrm{m}\right)$, circles are IRAC (3.6, 4.5, 5.8, $8 \mu \mathrm{m})$, and squares are MIPS $(24 \mu \mathrm{m})$. A gray line is extended from $K_{s}$ to guide the eye, representing an extrapolated Rayleigh-Jeans slope. If the object is a star, there is no excess at $K_{s}$, no significant reddening, and the star is earlier than mid-M, this would be the approximate location of the photosphere. 2-1066 shows a clear possible IR excess; a very weak excess appears to be present at $24 \mu \mathrm{m}$ for 7-7604.

\subsection{Identifying Low-mass Binaries}

In Figure 11, we show a $J H K_{s}$ color-color diagram plotting all of the 82 eclipsing binary systems for which good quality photometry are found in the 2MASS point-source catalog. Also indicated are the regions encompassed by the main sequence and the giant branch. The nine young binary candidates are highlighted with star symbols as before.

From the figure we can see that, with the exception of the two contact binaries, 5-12446 and 10-10597, all of the previously discussed candidate young systems also have 2MASS colors that - assuming no major reddening — are consistent with M-dwarf primaries. These systems are therefore consistent with being both young and low mass.

In addition to the candidate young systems already discussed, we also mark on the diagram the five other systems that fit in or near the M-dwarf region of the plot, but are not necessarily young or associated with Orion: 0-3542, 0-9653, 2-822, 5-5767, and 11-7037, of which three (0-3542, 0-9653, and 11-7037) are classified as "close" systems. These five systems are also broken out at the beginning of Table 1 and are discussed in Sections 5.4.2 and 5.4.4.

Dereddening any of these systems by the mean assumed reddening, indicated on the plot, would make little difference to their status. For the young-system candidates, it only brings the sources closer to the main sequence. However, it remains possible that some sources are either obscured by tight localized regions of interstellar dust, or intrinsically reddened objects. The small IR excesses in the Spitzer data for 2-1066 (Section 5.2.5), and possibly 7-7604, may be indicative of intrinsic reddening. It should be noted that the possibility of unresolved source confusion remains, which could also lead to a distortion of the measured colors.

\subsection{Discussion}

In the previous sections (Sections 5.2 and 5.3), we discussed the approaches employed for identifying particular sources of interest in the eclipsing binary sample. We identified nine PMS candidate systems (0-7220, 2-1066, 2-1144, 4-5331, 5-12446, 


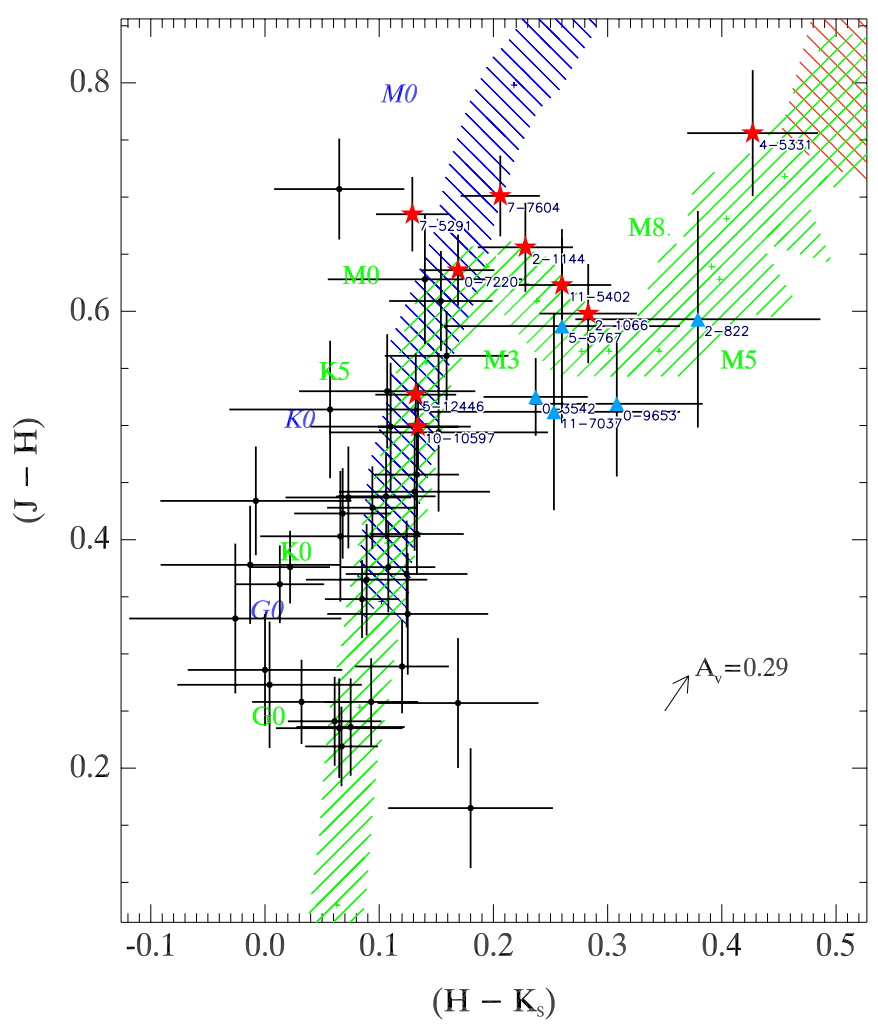

Figure 11. Color-color diagram for the new binaries based on 2MASS colors (Section 5.3). The green hatched region (bottom left to top right in the printed journal) marks the main sequence, the blue hatched region (middle left to top middle in the printed journal) marks the giant branch, and the red hatched region (top right in the printed journal) marks the beginning of the L-dwarf regime. The assumed mean reddening vector for the field is indicated. The candidate young binary systems are marked with a star symbol as in previous figures. In addition, five other potential low-mass binary systems are marked with triangles on the basis of their location in the diagram.

(A color version of this figure is available in the online journal.)

7-5291, 7-7604, 10-10597, and 11-5402), of which two (512446 and 10-10597) appear to be contact binary systems, one is also categorized as "close" but is somewhat anomalous (75291), and the remainder are likely low-mass systems. (Contact binaries are not normally found as late as M-type-see, e.g., Rucinski 2004; Bilir et al. 2005.) We also identified three notable very red contact binaries (0-3542, 0-9653, and 117037). In addition, two more systems (not previously discussed) are particularly interesting: 11-8774, a contact binary with an exceptionally short period, and 9-2980, a detached system which appears to exhibit stellar pulsations. We summarize the results here and discuss some of the specific systems. Since there is significant overlap between the results from the analysis above, we break down the specific sources identified into the four categories below, which are reflected at the beginning of Table 1 .

\subsubsection{Young Low-mass Binary Candidates}

Six of the nine young candidate systems we found also fell into the category of candidate low-mass (M0 or later) systems: 0-7220, 2-1066, 2-1144, 4-5331, 7-7604, and 11-5402. These specific systems are discussed in more detail below. The remaining three are close binaries, discussed in Section 5.4.3 (though 7-5291 may also qualify as low mass).

$0-7220$. Presents an unusual light curve. The regular variation outside the primary and (small, $\sim 1 \%$ ) secondary eclipses is comparable with the depth of the primary eclipse itself (see Figure 4(a)), and its phase is not consistent with ellipsoidal variation. It may be attributable to star spots: either one spot system with the star rotating at twice the orbital period, or more likely, two spot systems, separated by $\approx 180^{\circ}$ in longitude, rotating synchronously with the 0.680 day orbit. This would also be consistent with the long-term variation of the out-of-eclipse modulation during the length of the observations, which can be seen from the gray scale in the light curve plot (Figure 4(a)).

2-1066. Although coverage for this object was good, only one eclipse was observed (Figure 4(a), bottom right), so the period is indeterminate. The eclipse is flat-bottomed, making it a noteworthy source for further observations. Out-of-eclipse variation is on the level of $\approx 4 \%$. It is also notable for its significant apparent IR excess, with $K_{s}-[24]=4.75 \pm$ 0.06 mag (Section 5.2.5 and Figure 10), which is suggestive of a disk (although it may also be attributable to the secondary companion).

2-1144. Displays a similar light curve to 0-7220, though a secondary eclipse is not clearly evident in the data, and the outof-eclipse variation peaks only once per orbit. Again, this is not consistent with ellipsoidal variation and is likely attributable to star spot/hot spot variation with the stellar rotation period synchronous with the 0.554 day orbital period.

4-5331. Only part of one eclipse was captured for this object (Figure 4(b)), and therefore the period is not constrained, although coverage was good. The rest of the light curve is variable with an amplitude of $\sim 0.15 \mathrm{mag}$, with no clear period (Figure 5(a)). It is particularly noticeable for its very red colors, located as late as M9 in Figure 11; it is likely significantly reddened since if it had such a low mass it would appear much fainter at the distance of Orion. No coverage was found in the Spitzer data, but this reddening could be intrinsic, perhaps due to a disk. Though it seems likely that a stellar eclipse is the best explanation, it remains possible that the sudden drop in brightness is not in fact an eclipse from a binary companion, but that 4-5331 is a TTS, perhaps a "dipper"-like CTTS similar to 9-6886, shown in Figure 15(d) (Section 6).

7-7604. Shows a small but significant level of flaring consistent with its proposed young age and a low mass - hence the apparent discontinuity in the folded light curve (Figure 4(c)). The object shows a possible very weak IR excess in the Spitzer data at $K_{s}$ only, with $K_{s}-[24]=2.00 \pm$ $0.11 \mathrm{mag}$ and [3.6] $-[24]=1.79 \pm 0.13 \mathrm{mag}$ (Table 2 and Figure 10). 7-7604 lies closer to the giant branch than the main sequence in the $J H K_{s}$ color-color diagram (Figure 11), but if it is intrinsically reddened, this would bring its true color closer to the $\approx \mathrm{M} 0$ main-sequence region. The apparent broadness of the eclipses suggests quite a closely separated system, with significant tidal distortion. Similarly to 7-5291 (see below, Section 5.4.3), its short 2.07 day period argues against it being a giant system, since a giant would be larger than the implied orbital separation.

11-5402. Shows clear, roughly sinusoidal out-of-eclipse variation (Figure 4(e)), again most likely due to star spots given the phase of the variation relative to the eclipse. Only one eclipse was observed for the object. The eclipse has good coverage despite being narrow, and is shown in more detail in Figure 12, but the period of the binary is therefore not unambiguously determined. We take the period of the out-of-eclipse variations as the best guess of the orbital period, on the assumption of star spots corotating with the orbital period as for 0-7220 and 2-1144 (though the period here is longer, at 5.5 days). 


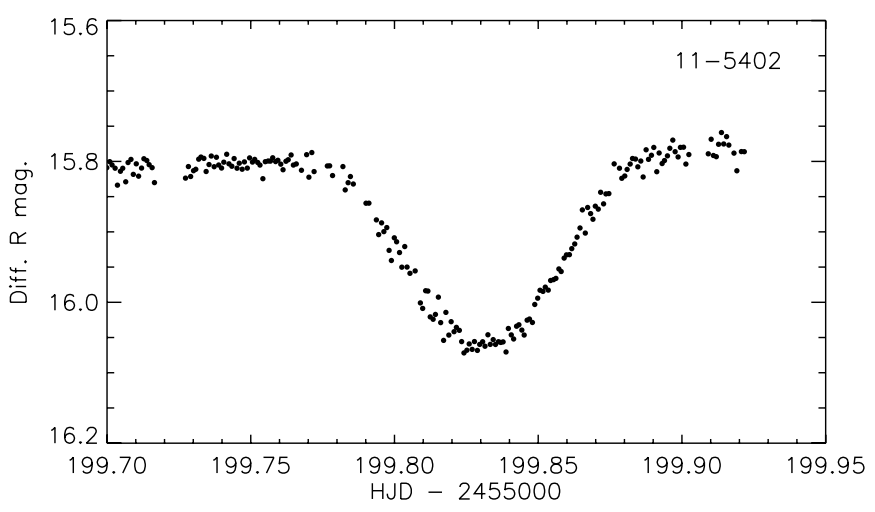

Figure 12. Stretched view of the light curve for 11-5402, showing the single eclipse observed for the object. The period for this system is estimated from the periodic out-of-eclipse variation (see Section 5.4.1). Error bars are omitted for clarity.

\subsubsection{Other Low-mass Binary Candidates}

We identified two other detached binary systems as low-mass candidates on the basis of the 2MASS $J H K_{s}$ color-color selection (Figure 11, Section 5.3). Given their distance estimates, and the fact that they do not appear in the PMS region of the color-magnitude diagram (Figure 6), they are most likely background systems unconnected with the Orion association.

2-822. Shows a straightforward grazing-transit-detached light curve, with out-of-eclipse variation suggesting longitudinal temperature variation on one of the components, synchronous with the 0.9204 day orbital period (Figure 4(a)). The $J H K_{S}$ colors suggest a spectral type of $\approx \mathrm{M} 5 \mathrm{~V}$ (though see the caveat below). Dereddening by the assumed mean reddening vector makes little difference to this estimation; dereddening by a greater amount would move the system off the main sequence, unless it is a late F-dwarf system, which would require a rather extreme reddening $\left(A_{V} \approx 5 \mathrm{mag}\right)$.

5-5767. Only two grazing eclipses were detected for this detached system, leaving a "factor of two" ambiguity in the period, which is resolved by the periodic out-of-eclipse variation, if we assume that it is synchronous with the orbital period. Folding on the resulting 2.105 day period suggests that both are primary (or both secondary) eclipses. From the phase coverage (Figure 4(b)), we see that the other eclipse was missed, assuming it would have fallen at phase 0.5 . The out-of-eclipse variation appears to be caused by star spots. The $J H K_{s}$ colors suggest a spectral type of $\approx \mathrm{M} 3 \mathrm{~V}$, unless it is a heavily reddened Gtype system, with $A_{V} \approx 2.5 \mathrm{mag}$. Again, see the caveat below, however.

In both cases, we note that given the sizes of their respective 2MASS measurement errors, the spectral type estimates are quite uncertain. The same two systems are labeled in the color-magnitude diagram in Figure 6. At face value, their $R-K_{s}$ values suggest somewhat earlier spectral types, around late $\mathrm{K}$ to $\mathrm{M} 0$ depending on reddening. However, the difference in epochs for the $R$ and $K_{s}$ observations leads also to large errors in $R-K_{s}$, as reflected in the error bars; given the errors in both the color-magnitude and the color-color diagrams, the measurements are not inconsistent. The true spectral types likely lie somewhere between the two values, around early-M-type. Follow-up spectroscopy is needed to provide a better estimate.

\subsubsection{Young "Close” Binary Candidates}

The majority of the "close" type binary systems in our sample appear most likely to be contact binaries from the shape of their light curves. Two W UMa systems are identified in our binary sample as candidate young 25 Ori/OB1a association members. It remains possible that their colors are distorted by unresolved third sources within the PSF, which could confuse their apparent young status, but if their ages are in indeed in the 7-10 Myr range, then there would be significant constraints on the mechanisms by which they could have formed, and they may merit further investigation. A third candidate young "close" system is less likely to be a contact binary, but shows some unusual properties.

5-12446. Shows a straightforward W UMa type light curve (Figure 4(c)), with period 0.23663 days. This system is an outlier in proper motion space, with a proper motion of $30.0 \pm 3.8 \mathrm{mas} \mathrm{yr}^{-1}$ in right ascension (Figure 9). It is probably an interloper; given its location somewhat below the cluster sequence in Figure 6, it could also be an unrelated system with a mass ratio near unity that has been shifted upward in the color-magnitude diagram due to the resulting luminosity enhancement, as discussed in Section 5.2.1. The expected location of such contact systems at young ages with respect to single-star isochrones is uncertain, however, and the system's estimated distance $(317 \pm 41 \mathrm{pc})$ is extremely close to that of the 25 Ori cluster. Even if this is an underestimate due to a near-unity mass ratio (Section 5.2.2), it may still lie in the range of the broader Orion association. The system was previously identified as a candidate variable star by Kraus et al. (2007).

7-5291. This object presents a peculiar light curve. Classified as a "close" type system under our definition, it has the appearance of a contact binary system, but with an unusual form. Assuming the two minima are indeed eclipses, then the width of the eclipses alone would suggest anything from a contact to a semi-detached system. However, the maxima and minima are rather sharp, and the primary ingress and egress are somewhat asymmetric. The period of the system, 3.62 days, is much longer than that expected for a W UMa system, and appears as an outlier in Figure 13. The 2MASS $J H K_{s}$ colors are consistent with a midto late-K-type giant or possibly a late-K- or M0-dwarf primary (see Figure 11), but the period does not allow for either of these: a dwarf system would not even be significantly tidally distorted at such an orbital separation $\left(\approx 10 R_{\odot}\right)$, and therefore could not be near contact; a giant would be larger than the orbital separation. This suggests a subgiant-sized primary.

One possibility is that distorted shape of the light curve results from a semi-detached (accreting) system with a disk. A semi-detached system would also be more consistent with the marked difference between the two eclipse depths, indicative of a relatively large temperature difference. If we assume that the system cannot be a contact binary owing to its period, then using the alternative (detached) distance calibration puts it at a distance of $268 \pm 64 \mathrm{pc}$, which is $\approx 1 \sigma$ from the 25 Ori/OB1a distance. A $10 \mathrm{Myr}$ old inflated young low-mass primary, however, still would not be large enough. An alternative explanation for the light curve may be periodic occultation by a warped disk, in an analogue to AA Tau (Bouvier et al. 2003), similar to the objects reported by Morales-Calderón et al. (2011). The available (single-epoch) Spitzer photometry of this object (Figure 10, Section 5.2.5), however, shows at most only a very marginal IR excess at $24 \mu \mathrm{m}$, with $K_{s}-[24]=0.84 \pm 0.12$ and $[3.6]-[24]=0.73 \pm 0.13 \mathrm{mag}$ (Table 2). The estimated spectral type is on the border between $\mathrm{K}$ and $\mathrm{M}$, however, and source confusion at the relatively low spatial resolution of the MIPS data is a potential concern. Additional follow-up is needed to 


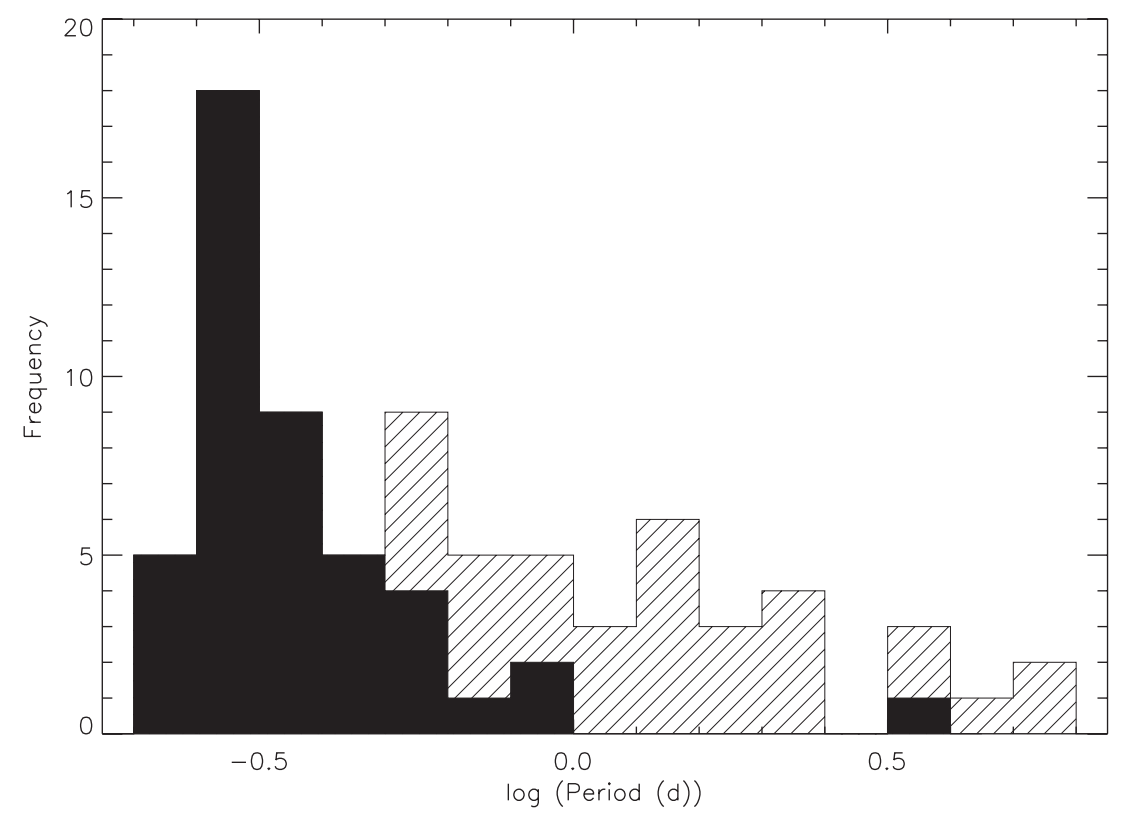

Figure 13. Orbital period histogram for all eclipsing binaries for which a period estimate could be found (Section 5.4.4). The solid regions indicate the fraction that is "close" (probably mostly contact binary) sources; the hatched regions indicate the remaining detached sources. At the shortest end of the distribution is 11-8774, with among the shortest W UMa periods known (Section 5.4.4). The outlying "close" source with a long period at just above $\log ($ period) $=0.5$ is due to object 7-5291, which presents a somewhat unusual light curve (see Section 5.4.3).

better constrain the spectral type and $A_{V}$ and better understand this object.

10-10597. Again shows a straightforward W UMa type light curve (Figure 4(e)), with a period of 0.29953 days. Spitzer data were found for this object showing no IR excess; this is unsurprising, as we might expect any disk to be disrupted in such a system. Like 5-12446, this system also lies somewhat below the cluster sequence in Figure 6, with a similar distance estimate (348 $\pm 45 \mathrm{pc})$, so the same comments with regard to possible near-unity mass ratio may apply.

\subsubsection{Other Binaries of Interest}

Among the remaining binaries, we identified a further five that are not necessarily young or associated with Orion, but are noteworthy for other reasons.

$0-3542,0-9653$, and 11-7037. In the $J H K_{s}$ color-color diagram (Figure 11), while all the other low-mass (M0V and later) systems are classified as "detached" and lie on or very close to the main sequence, these three alone lie somewhat below the M-dwarf branch, and show W UMa like light curves. If they are genuine contact binaries and not near-contact or semi-detached systems, their location on the diagram suggests that they are either anomalously low-mass systems (W UMa systems are not normally found as late as M-type-see, e.g., Rucinski 2004; Bilir et al. 2005), or probably more likely, particularly reddened W UMa systems $\left(A_{V} \approx 2-3 \mathrm{mag}\right)$ of earlier spectral type. In either case they may be interesting for further investigation. Given their unusual colors, we note that they lie outside the formal validity range of the Eker et al. (2009) calibrations, and their respective distance estimates may be unreliable. 0-9653 was previously identified as a candidate variable star by Kraus et al. (2007).

9 -2980. Shows small but pronounced $\sim 0.01 \mathrm{mag}, \approx 45$ minute modulations superimposed on the eclipsing binary light curve. Stellar pulsations seem the most likely cause. The 2MASS colors of the source imply a spectral type of $\sim \mathrm{G} 0$, and the significant out-of-eclipse variations suggest both spot modulation and ellipsoidal variation indicative of a tidally distorted system. More observations are needed to sample the secondary eclipse.

11-8774. Figure 13 shows a histogram of the periods of all the binaries from Table 1, highlighting the distribution of the "close" type systems. W UMa systems have a known sharp cutoff in the period distribution at the short-period end below around 0.22 days, just below a peak in the distribution (see, e.g., Rucinski 2007). The cause of this cutoff remains largely unclear. We have not attempted to correct for the effects of the observing windowing function or magnitude completeness effects, but it is still clear that a significant number of our targets lie near the short-period end of this distribution, which is not currently well sampled (Rucinski 2007). In particular, the shortest-period source, 11-8774, has a period of $0.2156509 \pm 0.0000071$ days, putting it among the most rapidly orbiting W UMa systems known. 11-8774 also displays clear flat-bottomed primary eclipses, meaning the eclipses are total rather than grazing (Figure 14). This makes the object a particularly good target for constraining parameters via light curve modeling. The derived distance of 11-8774, $450 \pm 60 \mathrm{pc}$, may be too distant for the 25 Ori cluster and the larger Orion $\mathrm{OB} 1 \mathrm{a}$ association $(\approx 330 \mathrm{pc})$, but is consistent with that of the more distant $(\approx 440$ pc; Briceño et al. 2005; Brown et al. 1999) — and younger-OB1b association. It could perhaps be an outlier of the OB1b association, although at such a young age, it would be far from the main sequence, and the distance estimate would likely be systematically wrong. If, however, the system does lie within the general distance of the broader Orion association, it could constitute a young W UMa system. As such it would be useful in understanding and constraining the formation and evolution of such systems.

\section{CTTS SOURCES}

Another class of light curve found in our data is that of CTTSs. These young systems exhibit particularly large and irregular brightness modulations, thought to be related to pho- 


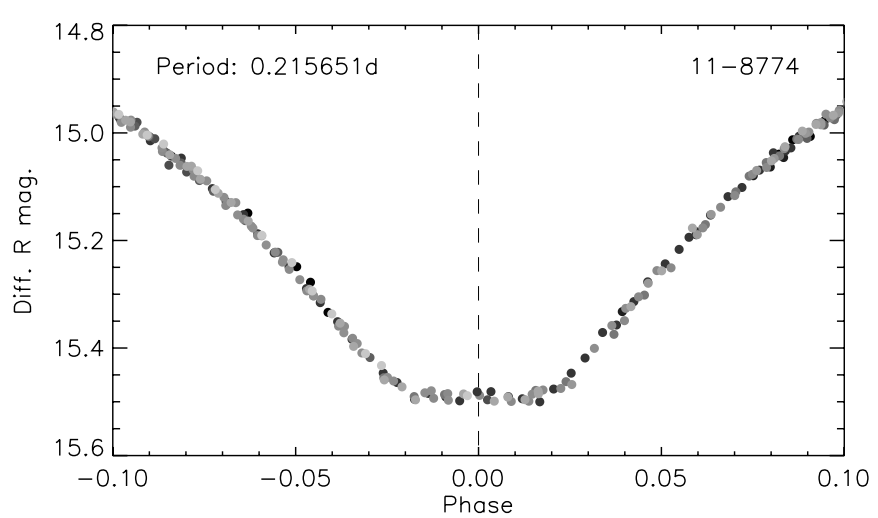

Figure 14. Stretched view of the folded light curve for the very short period W UMa system, 11-8774, centered on the primary eclipse (see Section 5.4.4.). The flat bottom of the eclipse can clearly be seen. The gray scale is again used to represent time of observation, running from dark gray at the beginning of the PTF Orion run to pale gray at the end of the run. The secondary eclipse (not shown) is also flat-bottomed. Error bars are omitted for clarity.

tospheric spots, short-lived accretion hot spots, and/or obscuration due to circumstellar dust (Herbst et al. 1994). On the basis of this variability, we identified visually 16 candidate CTTS light curves that showed irregular variability on scales of $\sim 1$ mag or more, listed in Table 3 . These include 14 candidates that are new to our knowledge, along with one known CTTS, CVSO 35 (8-10691), previously reported by Briceño et al. (2005; 2007), and one previously reported as a candidate WTTS, SDSS J052700.12+010136.8 (9-3485) (McGehee 2006). The light curves for all 16 of the sources are shown in Figure 15.

Figure 16 shows the location of the candidate CTTS sources on the same color-magnitude diagram as previously shown for the binaries (Figure 6). All clearly lie in the PMS region for the distance and mean reddening of the 25 Ori region.

Of the 16 CTTS sources, 10 were found with coverage in the Spitzer archive data (including the one known CTTS), and all show IR excesses suggestive of disk presence. The SEDs of these sources are shown in Figure 17. All the objects appear to have clear excesses, and for those where multi-band Spitzer data are available, the excess is seen in more than one band, lending credence to the identification of a disk excess. For the three where there are only $24 \mu \mathrm{m}$ detections, the apparent $24 \mu \mathrm{m}$ excess is very large $\left(K_{s}-[24]=3.52 \pm 0.06,5.45 \pm 0.06\right.$, and $4.69 \pm 0.06$, for sources 5-9166, 5-9411, and 7-3471, respectively), and seems to be real, although the uncertainty is larger than the formal error on the points because of the uncertainty in $A_{V}$ and spectral type.

1-3452. Seems to show a slight discontinuity between $J H K_{S}$ and the Spitzer IRAC bands (Figure 17). This may either be a result of the contribution from the blackbody function of the disk, or due to intrinsic variability between the epochs of 2MASS and Spitzer observations.

9-6886. Displays a light curve that appears flat for a substantial portion of the time, but with downward deviations of up to almost a magnitude (Figure 15(d)). This is reminiscent of "dipper" objects discussed by Morales-Calderón et al. (2011), for which AA Tau is a prototype (Bouvier et al. 2003). (These objects were previously alluded to in Section 5.4.3 with regard to object 7-5291.)

The new sources are spatially distributed fairly evenly across the PTF field of view, with no obvious clustering. This suggests that they are likely associated with the broader Orion OB1a association rather than with the 25 Ori group itself, which is at approximately the same distance.

\section{SUMMARY}

We have presented some of the initial results from the PTF Orion survey based on the most strongly variable light curves, relating to eclipsing binary systems and PMS stars, two of the main science objectives of the survey. We have identified 82 eclipsing binary systems, of which 37 are detached, and 45 are "close" (mostly contact binaries). Of the close systems, 11-8774 is among the shortest-period W UMa type systems known, lying in an as-yet poorly sampled region of W UMa parameter space. Furthermore, the distance estimate for this system puts it within the range of the Orion association, implying it may be very young: it may therefore be particularly interesting for constraining evolutionary models of contact binaries.

Among the eclipsing binaries, we find a total of nine candidate PMS systems, with implied ages of $\approx 7-10 \mathrm{Myr}$ if we assume Orion 25 Ori and/or Orion OB1a association membership. Their positions in the color-magnitude diagram, estimated distances (with the exception of one uncertain estimate), outof-eclipse variability, and proper motions (with the exception of one), are all consistent with their proposed youth and association membership, making them good candidate PMS binaries. Such systems are potentially valuable for constraining evolutionary models of both binary systems and individual stars. Furthermore, two of these systems are potentially young contact binaries. If these are genuinely as young as $7-10 \mathrm{Myr}$, this could have significant implications regarding the timescales of mechanisms by which they could have formed.

A number of the binary systems are also candidate lowmass systems, apparently with M-dwarf primaries; this is also a regime that remains poorly sampled to date. With the exception of the two contact systems (which are not expected at such late spectral types), most, if not all, of the above-mentioned candidate young systems appear to have low-mass primaries based on their 2MASS colors. We also find an additional two detached systems whose $J H K_{s}$ colors are suggestive of low mass, but with distance estimates more consistent with being background stars, and three contact (or near-contact) systems that appear to be unusually reddened.

Some of the systems show somewhat unusual light curves. One in particular, 7-5291, has the appearance of a distorted contact or near-contact binary curve, but has a much longer period than is found for contact binaries, and its position on the color-magnitude diagram is consistent with a young age at the distance of Orion. One possible explanation is that it is a semidetached system with an inflated PMS primary, perhaps with a disk - although the available Spitzer data do not seem to show a clear excess. Assuming it is not a contact binary system gives it a distance estimate nearer the 25 Ori/Orion OB1a association. Alternatively, the light curve might be explained by a star being occulted by a warped disk, in an analogue of AA Tau (Bouvier et al. 2003) and the "dipper" systems identified by MoralesCalderón et al. (2011). Further data and analysis are needed for this object.

One of the detached systems, 9-2980 appears to exhibit stellar pulsations superimposed on the eclipsing binary light curve. The pronounced ellipsoidal variation suggests a fairly closely separated system. Further data are needed for coverage of the secondary eclipse, but the pulsations could potentially allow 
Table 3

Candidate CTTSs: Sources with Irregular Light Curves and $\Delta R \sim 1.0$ mag or More

\begin{tabular}{|c|c|c|c|c|c|c|c|c|c|c|c|}
\hline \multirow[t]{2}{*}{ ID } & \multirow[t]{2}{*}{$\begin{array}{c}\alpha \\
(\operatorname{deg})\end{array}$} & \multirow[t]{2}{*}{$\begin{array}{c}\delta \\
(\operatorname{deg})\end{array}$} & \multirow[t]{2}{*}{ 2MASS } & \multirow{2}{*}{$\begin{array}{c}\mathrm{PM} \alpha \\
\left(\mathrm{mas} \mathrm{yr}^{-1}\right)\end{array}$} & \multirow{2}{*}{$\begin{array}{c}\begin{array}{c}\mathrm{PM} \delta \\
\left(\mathrm{mas} \mathrm{yr}^{-1}\right)\end{array} \\
{[4.5]} \\
(\mathrm{mag})\end{array}$} & \multirow{2}{*}{$\begin{array}{c}\begin{array}{c}R_{\mathrm{med}}{ }^{\mathrm{d}} \\
(\mathrm{mag})\end{array} \\
{[5.8]} \\
(\mathrm{mag})\end{array}$} & \multirow{2}{*}{$\begin{array}{c}\begin{array}{c}\Delta R \\
(\mathrm{mag})\end{array} \\
\begin{array}{c}{[8]} \\
(\mathrm{mag})\end{array}\end{array}$} & \multirow{2}{*}{$\begin{array}{c}\begin{array}{c}\sigma_{R} \\
(\mathrm{mag})\end{array} \\
\begin{array}{c}{[24]} \\
(\mathrm{mag})\end{array}\end{array}$} & \multirow{2}{*}{$\begin{array}{c}J \\
(\mathrm{mag})\end{array}$} & \multirow{2}{*}{$\begin{array}{c}\begin{array}{c}H \\
(\mathrm{mag})\end{array} \\
\begin{array}{c}{[3.6]-[24]} \\
(\mathrm{mag})\end{array}\end{array}$} & \multirow[t]{2}{*}{$\begin{array}{c}K_{s} \\
(\mathrm{mag})\end{array}$} \\
\hline & & & & & & & & & & & \\
\hline $1-3452$ & 81.07590 & 2.28890 & $05241824+0217200$ & $\begin{aligned} 7.0 & \pm 3.9 \\
10.36 & \pm 0.05\end{aligned}$ & $\begin{aligned} 2.7 & \pm 3.9 \\
9.86 & \pm 0.05\end{aligned}$ & $\begin{array}{c}17.33 \\
9.56 \pm 0.05\end{array}$ & $\begin{array}{c}1.08 \\
9.17 \pm 0.05\end{array}$ & $\begin{array}{c}0.23 \\
6.91 \pm 0.05\end{array}$ & $\begin{array}{r}13.60 \pm 0.02 \\
4.96 \pm 0.05\end{array}$ & $\begin{aligned} 12.59 & \pm 0.02 \\
3.45 & \pm 0.07\end{aligned}$ & $11.88 \pm 0.03$ \\
\hline $2-8756$ & 81.42163 & 2.81835 & $05254119+0249059$ & $\begin{array}{c}0.3 \pm 3.9 \\
\ldots\end{array}$ & $\begin{array}{c}-7.7 \pm 3.9 \\
\ldots\end{array}$ & $\begin{array}{c}16.33 \\
\ldots\end{array}$ & $\begin{array}{l}1.09 \\
\ldots\end{array}$ & $\begin{array}{l}0.27 \\
\ldots\end{array}$ & $\begin{array}{c}13.59 \pm 0.03 \\
\ldots\end{array}$ & $\begin{array}{c}12.86 \pm 0.03 \\
\ldots\end{array}$ & $12.35 \pm 0.03$ \\
\hline $4-2045$ & 82.63182 & 2.05147 & 05303164+0203051 & $\begin{array}{c}3.8 \pm 4.0 \\
9.86 \pm 0.05\end{array}$ & $\begin{aligned}-0.3 & \pm 4.0 \\
9.58 & \pm 0.05\end{aligned}$ & $\begin{array}{c}14.49 \\
9.39 \pm 0.05\end{array}$ & $\begin{array}{c}1.13 \\
8.62 \pm 0.05\end{array}$ & $\begin{array}{c}0.30 \\
5.29 \pm 0.05\end{array}$ & $\begin{aligned} 11.97 & \pm 0.02 \\
5.19 & \pm 0.05\end{aligned}$ & $\begin{aligned} 11.02 & \pm 0.02 \\
4.57 & \pm 0.07\end{aligned}$ & $10.48 \pm 0.02$ \\
\hline $5-9166$ & 82.87327 & 2.68835 & $05312959+0241183$ & $\begin{array}{c}-0.8 \pm 4.0 \\
\ldots\end{array}$ & $0.2 \pm 4.0$ & 15.88 & 0.92 & $\begin{array}{c}0.24 \\
8.35 \pm 0.05\end{array}$ & $\begin{aligned} 13.19 & \pm 0.03 \\
3.52 & \pm 0.06\end{aligned}$ & $\begin{array}{c}12.39 \pm 0.03 \\
\ldots\end{array}$ & $11.87 \pm 0.03$ \\
\hline $5-9411$ & 82.97474 & 2.70859 & $05315396+0242310$ & $\begin{array}{c}6.1 \pm 3.8 \\
\ldots\end{array}$ & $\begin{array}{c}-4.4 \pm 3.8 \\
\ldots\end{array}$ & 17.55 & 1.72 & $\begin{array}{c}0.45 \\
7.73 \pm 0.05\end{array}$ & $\begin{aligned} 14.97 & \pm 0.05 \\
5.45 & \pm 0.06\end{aligned}$ & $\begin{array}{c}13.99 \pm 0.04 \\
\ldots\end{array}$ & $13.18 \pm 0.04$ \\
\hline 7-3471 & 80.97543 & 1.13702 & $05235410+0108128$ & $\begin{array}{c}0.5 \pm 3.9 \\
\ldots\end{array}$ & $\begin{array}{c}-10.9 \pm 3.9 \\
\ldots\end{array}$ & 16.03 & 1.14 & $\begin{array}{c}0.20 \\
7.60 \pm 0.05\end{array}$ & $\begin{array}{r}13.50 \pm 0.03 \\
4.69 \pm 0.06\end{array}$ & $\begin{array}{c}12.76 \pm 0.03 \\
\ldots\end{array}$ & $12.29 \pm 0.03$ \\
\hline $8-6391$ & 81.26950 & 1.34760 & $05250468+0120509$ & $\begin{array}{l}-0.4 \pm 3.9 \\
10.06 \pm 0.05\end{array}$ & $-1.6 \pm 3.9$ & $\begin{array}{c}15.37 \\
9.21 \pm 0.05\end{array}$ & 0.90 & $\begin{array}{c}0.24 \\
5.15 \pm 0.04\end{array}$ & $\begin{array}{r}12.46 \pm 0.03 \\
5.91 \pm 0.05\end{array}$ & $\begin{aligned} 11.67 & \pm 0.02 \\
4.92 & \pm 0.07\end{aligned}$ & $11.06 \pm 0.02$ \\
\hline $8-10691^{a}$ & 81.44118 & 1.76381 & $05254589+0145500$ & $\begin{array}{c}6.1 \pm 3.9 \\
9.74 \pm 0.05\end{array}$ & $\begin{aligned}-1.7 & \pm 3.9 \\
9.42 & \pm 0.05\end{aligned}$ & $\begin{array}{c}13.77 \\
8.94 \pm 0.05\end{array}$ & $\begin{array}{c}1.60 \\
7.58 \pm 0.05\end{array}$ & $\begin{array}{c}0.53 \\
4.73 \pm 0.04\end{array}$ & $\begin{aligned} 11.46 & \pm 0.03 \\
5.62 & \pm 0.05\end{aligned}$ & $\begin{aligned} 10.76 & \pm 0.03 \\
5.01 & \pm 0.07\end{aligned}$ & $10.35 \pm 0.02$ \\
\hline $9-3150$ & 82.23882 & 0.99533 & $05285732+0059431$ & $\begin{array}{c}5.6 \pm 16.3 \\
\ldots\end{array}$ & $\begin{array}{c}-31.4 \pm 16.3 \\
\ldots\end{array}$ & $\begin{array}{c}18.70 \\
\ldots\end{array}$ & $\begin{array}{c}1.38 \\
\ldots\end{array}$ & $\begin{array}{c}0.30 \\
\ldots\end{array}$ & $\begin{array}{c}13.90 \pm 0.03 \\
\ldots\end{array}$ & $\begin{array}{c}13.26 \pm 0.03 \\
\ldots\end{array}$ & $12.89 \pm 0.03$ \\
\hline $9-3485^{\mathrm{b}}$ & 81.75044 & 1.02696 & $05270012+0101368$ & $2.3 \pm 3.9$ & $-3.2 \pm 3.9$ & 16.46 & 0.78 & 0.18 & $\begin{array}{c}13.18 \pm 0.02 \\
\ldots\end{array}$ & $\begin{array}{c}12.47 \pm 0.02 \\
\ldots\end{array}$ & $12.10 \pm 0.03$ \\
\hline $9-6886^{c}$ & 82.08428 & 1.35453 & $05282024+0121159$ & $\begin{array}{c}3.5 \pm 3.9 \\
\ldots\end{array}$ & $\begin{array}{c}-0.4 \pm 3.9 \\
\ldots\end{array}$ & $\begin{array}{c}15.07 \\
\ldots\end{array}$ & 0.71 & 0.22 & $\begin{array}{c}13.05 \pm 0.02 \\
\ldots\end{array}$ & $\begin{array}{c}12.31 \pm 0.02 \\
\ldots\end{array}$ & $12.06 \pm 0.02$ \\
\hline 9-10304 & 81.73075 & 1.67283 & $05265537+0140224$ & $\begin{aligned}-16.0 & \pm 3.9 \\
10.59 & \pm 0.05\end{aligned}$ & $\begin{aligned} 2.9 & \pm 3.9 \\
10.34 & \pm 0.06\end{aligned}$ & $\begin{array}{c}15.16 \\
10.24 \pm 0.06\end{array}$ & $\begin{array}{c}0.82 \\
9.76 \pm 0.06\end{array}$ & $\begin{array}{c}0.13 \\
6.08 \pm 0.05\end{array}$ & $\begin{aligned} 12.85 & \pm 0.03 \\
5.34 & \pm 0.05\end{aligned}$ & $\begin{array}{r}11.96 \pm 0.03 \\
4.51 \pm 0.07\end{array}$ & $11.42 \pm 0.02$ \\
\hline $10-2160$ & 82.72717 & 0.94125 & $05305452+0056286$ & $\begin{array}{c}5.4 \pm 4.0 \\
\ldots\end{array}$ & $\begin{array}{c}-1.5 \pm 4.0 \\
\ldots\end{array}$ & $\begin{array}{c}17.10 \\
\ldots\end{array}$ & $\begin{array}{c}0.76 \\
\ldots\end{array}$ & $\begin{array}{c}0.14 \\
\ldots\end{array}$ & $\begin{array}{c}13.34 \pm 0.03 \\
\ldots\end{array}$ & $\begin{array}{c}12.80 \pm 0.03 \\
\ldots\end{array}$ & $12.46 \pm 0.03$ \\
\hline $10-10515$ & 82.55167 & 1.80581 & $05301240+0148214$ & $\begin{array}{c}3.9 \pm 3.9 \\
11.52 \pm 0.06\end{array}$ & $\begin{array}{c}-1.7 \pm 3.9 \\
11.16 \pm 0.06\end{array}$ & $\begin{array}{c}16.47 \\
10.87 \pm 0.06\end{array}$ & $\begin{array}{c}1.29 \\
10.35 \pm 0.06\end{array}$ & $\begin{array}{c}0.27 \\
7.71 \pm 0.05\end{array}$ & $\begin{array}{r}13.69 \pm 0.03 \\
4.38 \pm 0.05\end{array}$ & $\begin{array}{r}12.79 \pm 0.02 \\
3.81 \pm 0.07\end{array}$ & $12.10 \pm 0.02$ \\
\hline $10-10679$ & 82.41315 & 1.82079 & $05293913+0149156$ & $\begin{array}{c}-3.8 \pm 3.9 \\
11.77 \pm 0.06\end{array}$ & $\begin{array}{c}5.0 \pm 3.9 \\
11.41 \pm 0.06\end{array}$ & $\begin{array}{c}16.86 \\
11.11 \pm 0.06\end{array}$ & $\begin{array}{c}0.38 \\
10.63 \pm 0.06\end{array}$ & $\begin{array}{c}0.09 \\
8.48 \pm 0.05\end{array}$ & $\begin{array}{r}13.72 \pm 0.02 \\
4.05 \pm 0.06\end{array}$ & $\begin{array}{r}13.03 \pm 0.03 \\
3.29 \pm 0.07\end{array}$ & $12.53 \pm 0.02$ \\
\hline $11-8549$ & 82.99254 & 1.66050 & $05315820+0139383$ & $\begin{array}{c}4.1 \pm 3.8 \\
\quad \cdots\end{array}$ & $\begin{array}{c}-1.6 \pm 3.8 \\
\ldots\end{array}$ & $\begin{array}{c}17.75 \\
\ldots \\
\end{array}$ & 0.96 & 0.21 & $\begin{array}{c}14.40 \pm 0.04 \\
\ldots\end{array}$ & $\begin{array}{c}13.72 \pm 0.04 \\
\ldots\end{array}$ & $13.13 \pm 0.04$ \\
\hline
\end{tabular}

Notes. See Section 6. 2MASS quality flag is "A" in all three bands for all sources (see Section 2.3). A description of how to construct general PTF catalog identifiers is given in Table 1.

${ }^{a}$ Known CTTS CVSO 35 (Briceño et al. 2005, 2007).

${ }^{\mathrm{b}}$ Reported as a candidate WTTS by McGehee (2006) (SDSS J052700.12+010136.8).

${ }^{c}$ Resembles the "dipper" objects discussed by Morales-Calderón et al. (2011)

${ }^{\mathrm{d}}$ See Section 4.2 for a discussion of zero-point accuracy. 

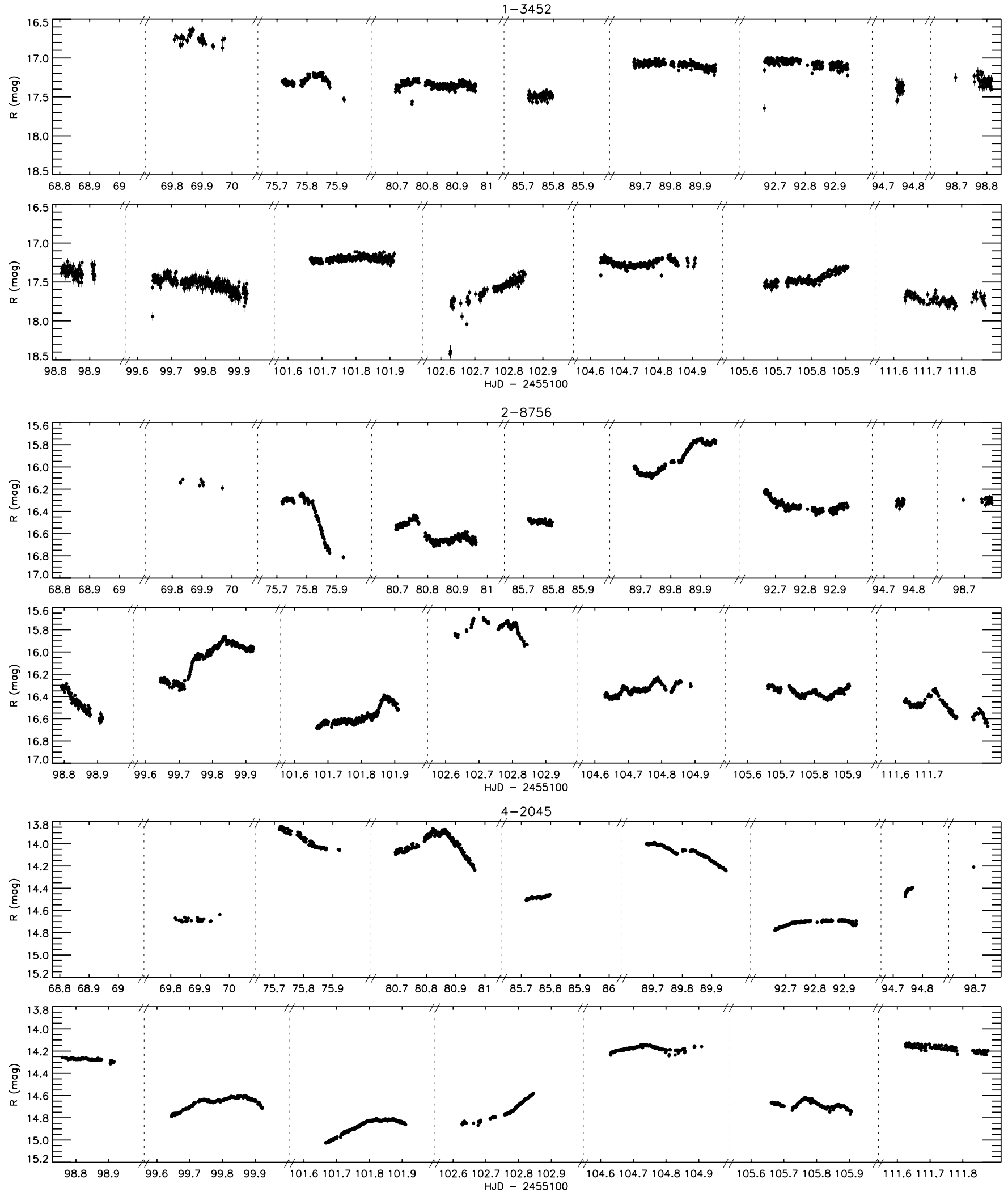

(a)

Figure 15. Light curves for the candidate CTTS (see Section 6). 8-10691 (c, center panel) is a known CTTS (Briceño et al. 2005). 9-3485 (d, top panel) was previously reported as a candidate weak-lined TTS by McGehee (2006). 9-6886 (d, center panel) resembles the "dipper" sources discussed by Morales-Calderón et al. (2011). 

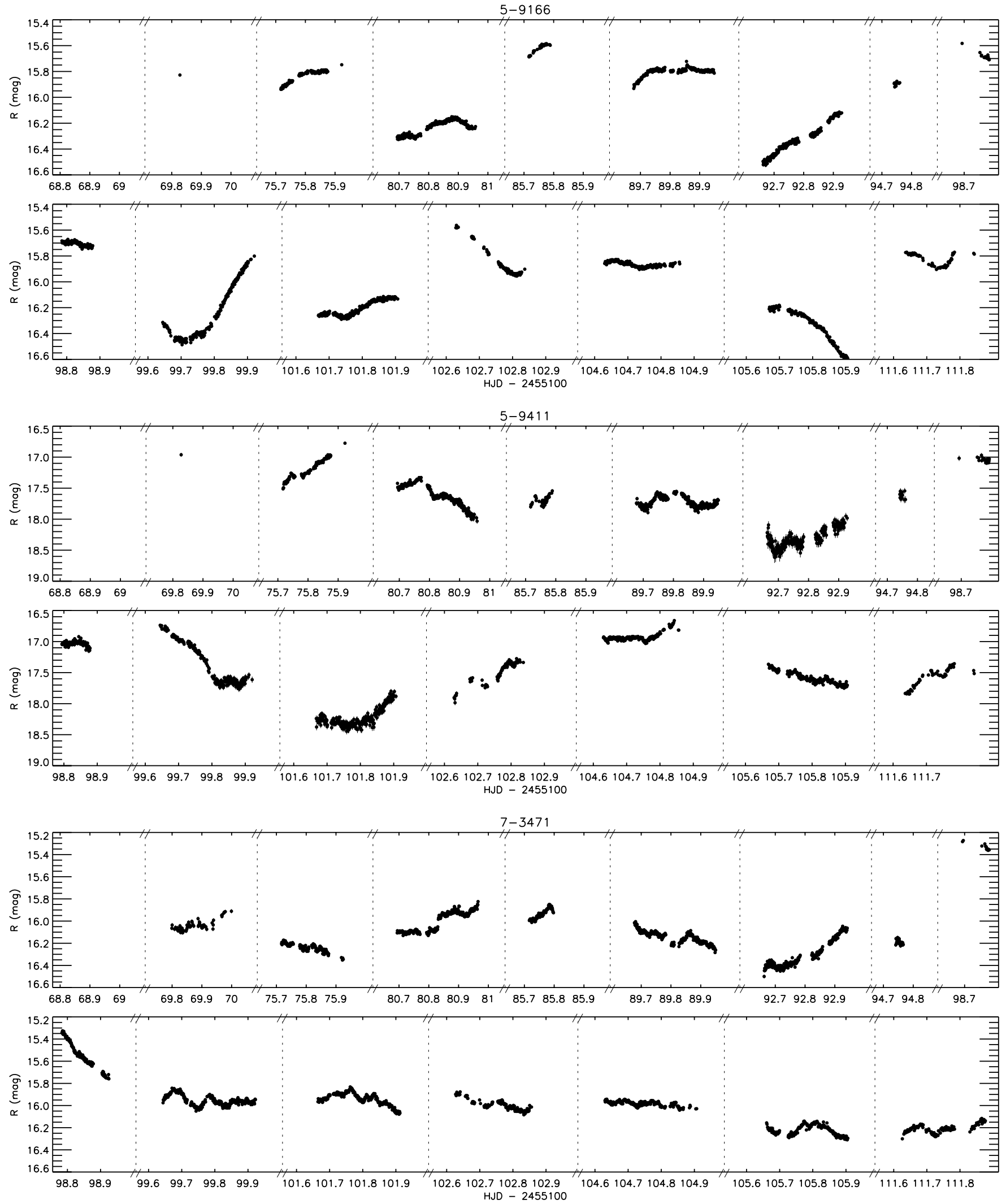

(b)

Figure 15. (Continued)

for valuable asteroseismological measurements of the system to complement those that can be obtained from standard light curve fitting and RV measurements.
Finally, we have identified 16 candidate CTTS systems, 14 of which are new to our knowledge, 1 of which is already known (8-10691, CVSO 35; Briceño et al. 2005, 2007), and 1 of which 

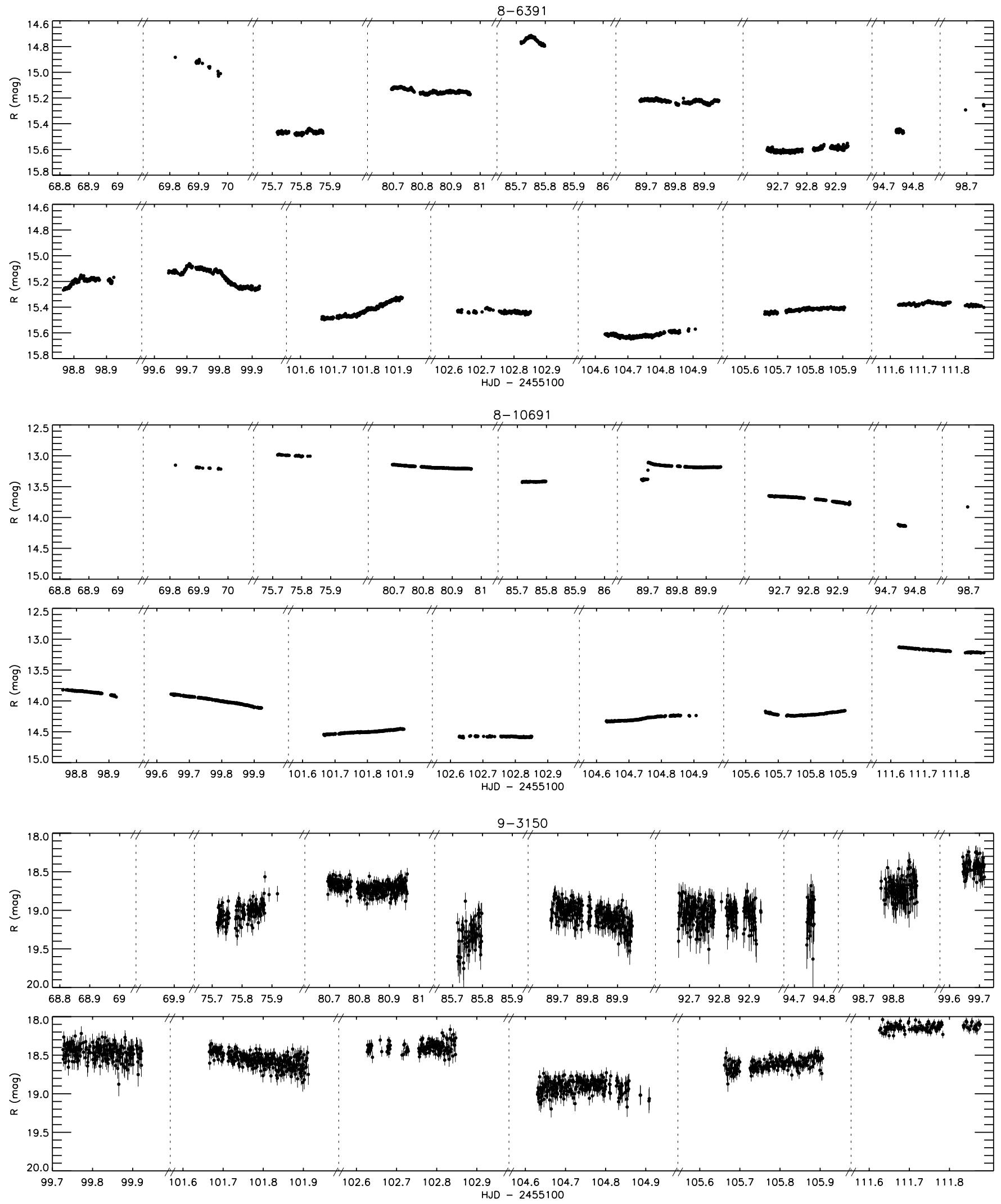

(c)

Figure 15. (Continued)

has previously been reported as a candidate WTTS (9-3485, J052700.12+010136.8; McGehee 2006). One of the new CTTSs, 9-6886, has the appearance of another "dipper" source.
Absolute photometric accuracy in the data set is estimated to be around $3 \%-5 \%(\approx 7 \%$ for chips 5 and 11$)$. The noise floor in the differential photometry sets in at a precision of around 

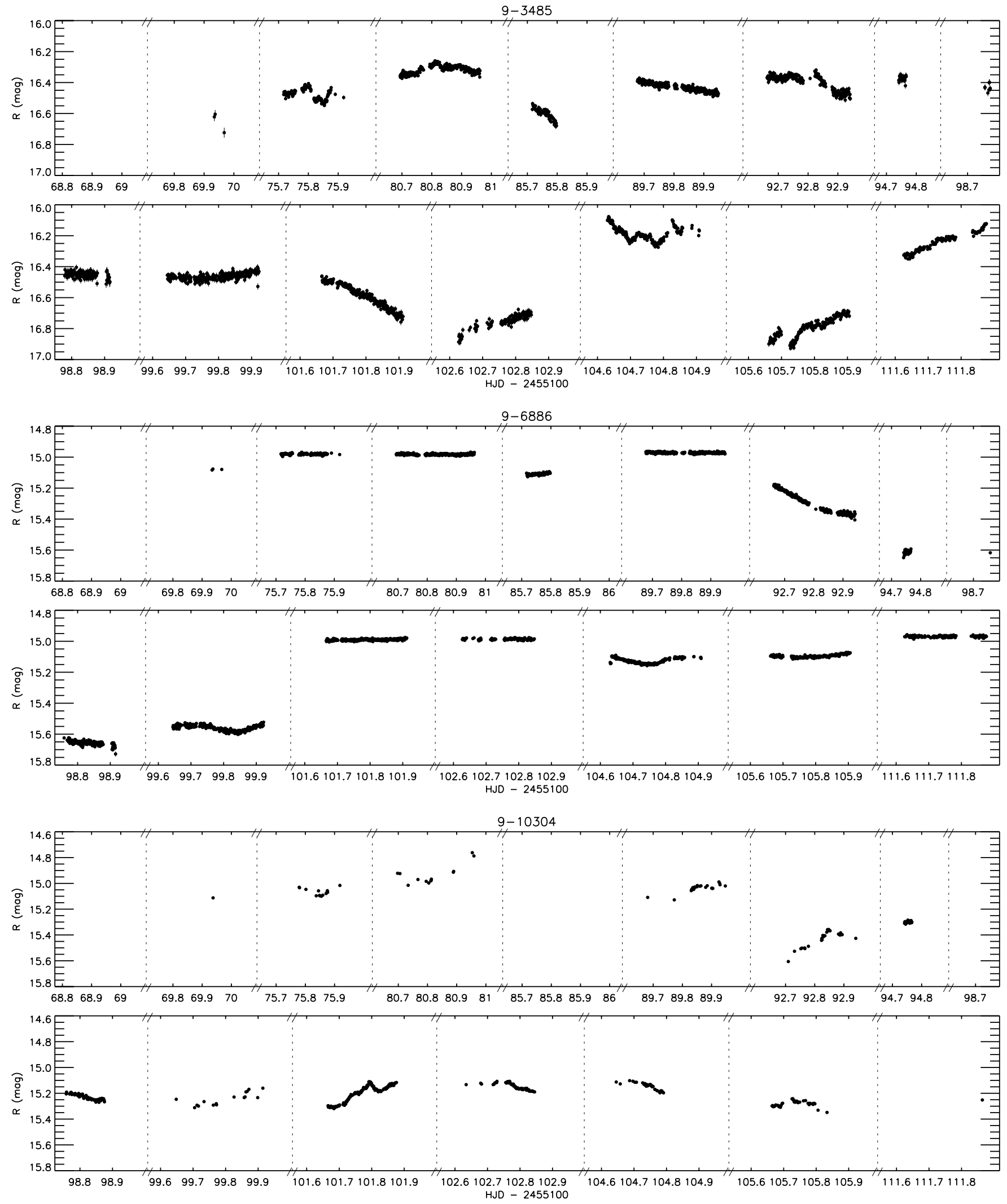

(d)

Figure 15. (Continued)

4 mmag for a single exposure, without any attempt at detrending. The differential photometry algorithm developed appears to be relatively robust against systematic trends in all but the worst cases, although, owing to known instrument issues at the time of these observations, detrending the data may reveal more light curves that could be of interest, particularly on chip 4.

The data reported here represent an initial cut of only the most strongly variable stars, but have yielded a wealth of 

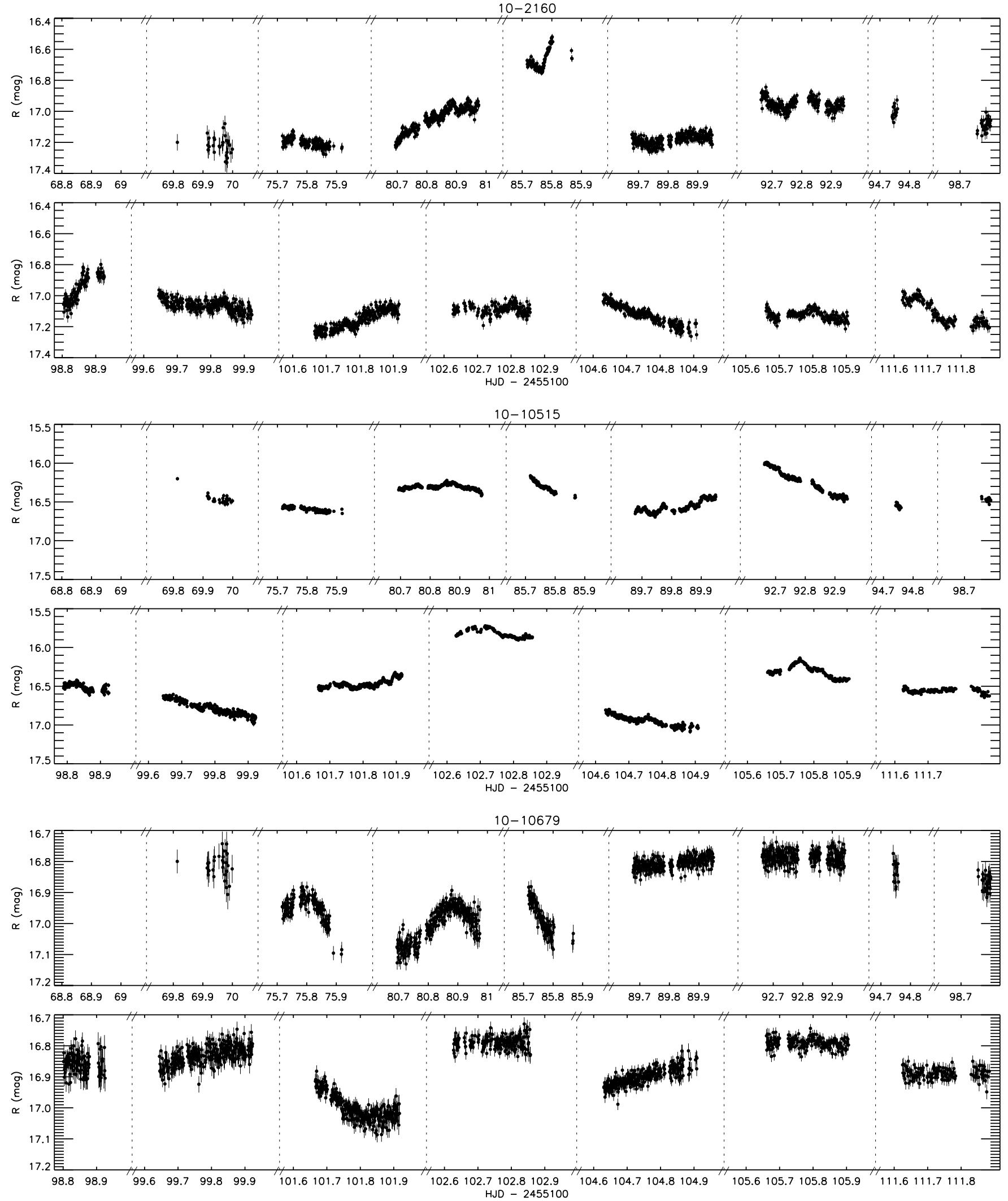

(e)

Figure 15. (Continued)

interesting data. Further follow-up data on the same field taken with PTF at the time of writing will enable confirmation of interesting sources, and help to distinguish persistent sources of variability (binaries, planets, etc.) from more transient sources (star spots, stellar activity, etc.). A finer search of the PTF Orion data at a lower variability threshold will explore a much larger sample of sources. Much remains to be mined in the data. 


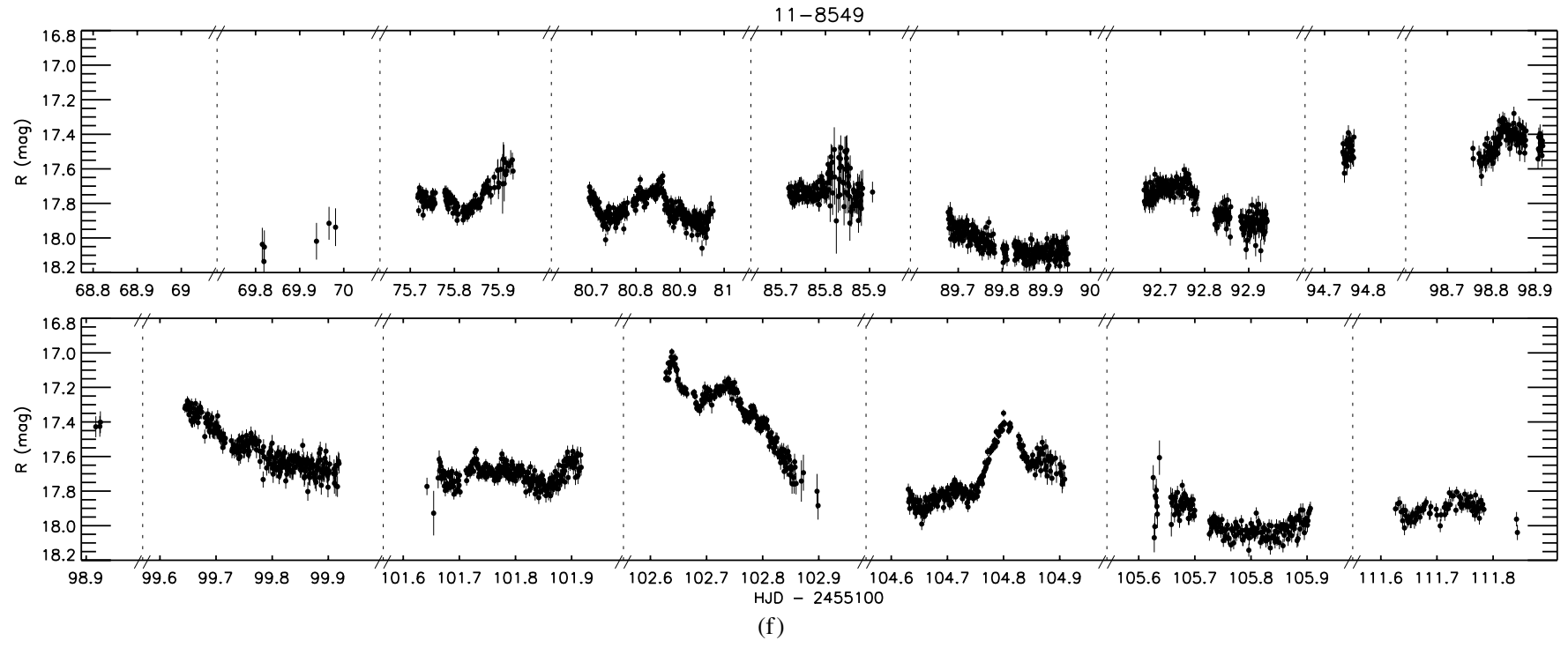

Figure 15. (Continued)

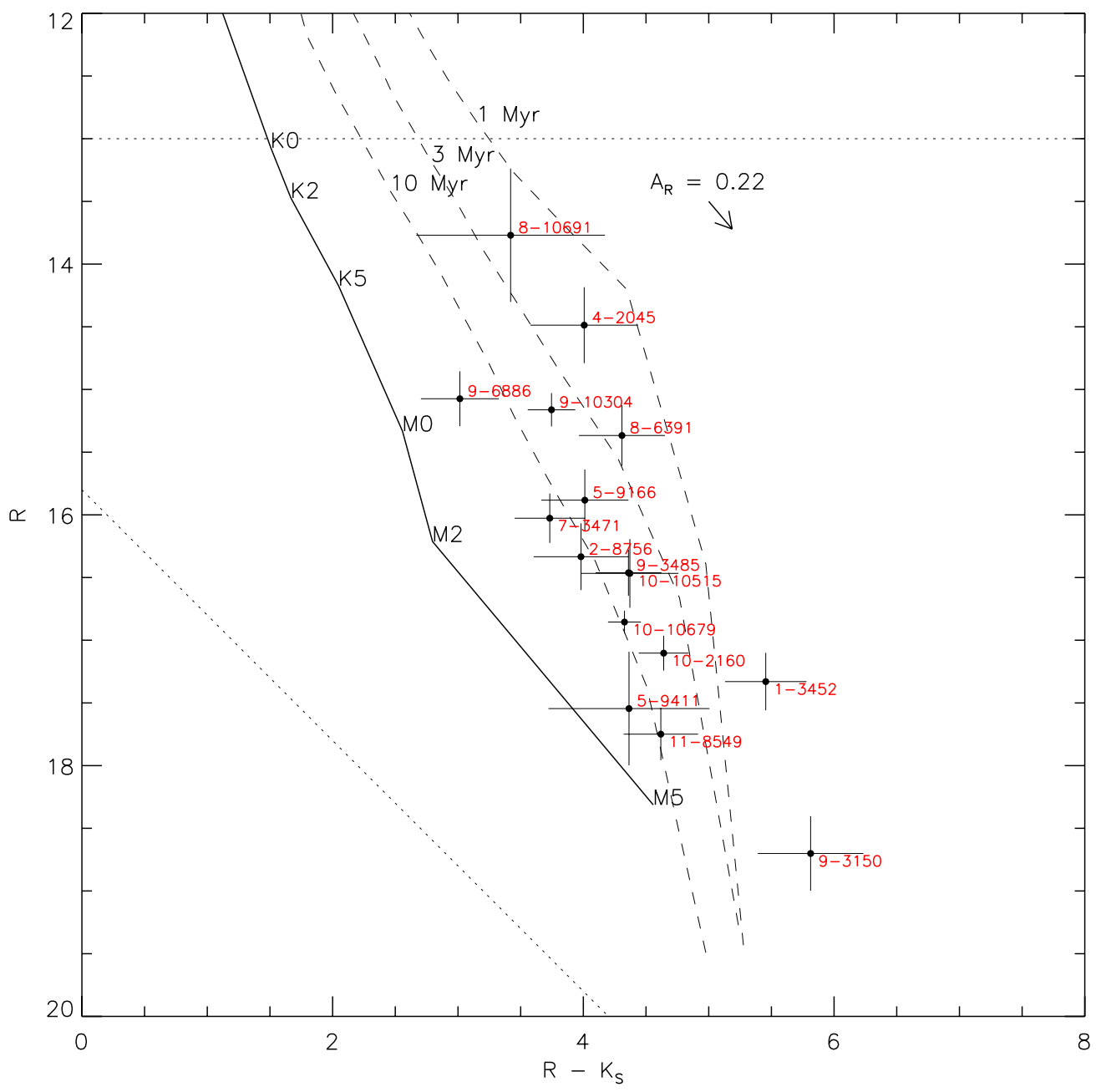

Figure 16. Color-magnitude diagram as for Figure 6, for the CTTS identified in the PTF Orion data matched against the 2MASS catalog (see Section 6). Errors in $R$ represent the standard deviation in the clipped (1st-99th percentile) standard deviation of the light curves to account for variability; errors in $K_{s}$ are taken to be the same in the absence of other information, added in quadrature with the intrinsic measurement errors in $K_{s}$.

(A color version of this figure is available in the online journal.) 


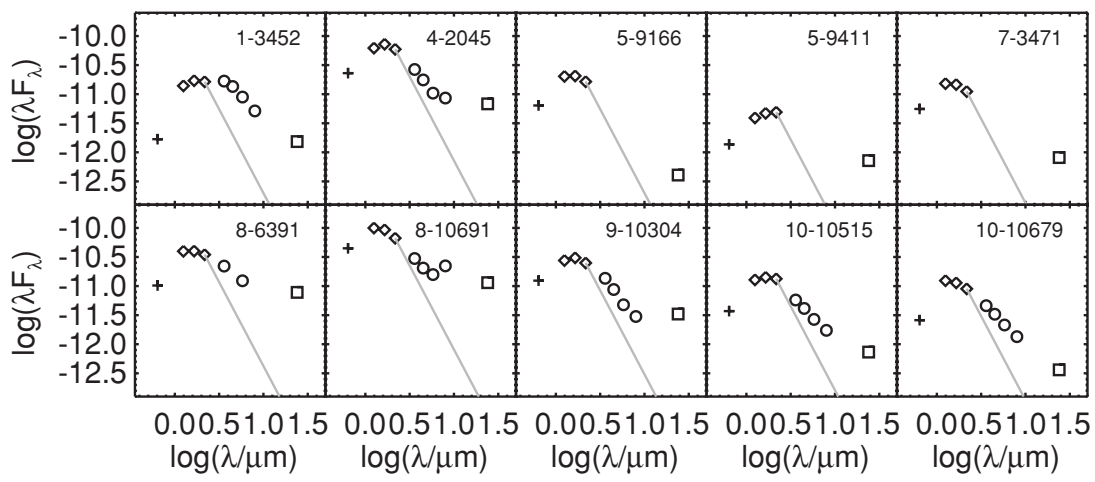

Figure 17. SEDs for the 10 CTTS-classified sources for which Spitzer archive data were available (nine new candidates and the known CTTS, 8-10691 (CVSO 35; Briceño et al. 2005; 2007)). Symbols are as for Figure 10, and the gray line again represents an extrapolated photosphere. All appear to have clear excesses, as expected for such sources.

The authors thank Cesar Briceño for providing the reference stars for zero-point calibration, and for his advice regarding field selection; and Tim Lister, Rachel Street, Andrej Prša, David Bradstreet, Ed Guinan, and Adam Krauss, for valuable discussions and input. S.B.C. wishes to acknowledge generous support from Gary and Cynthia Bengier, the Richard and Rhoda Goldman Fund, National Aeronautics and Space Administration (NASA)/Swift grant NNX10AI21G, NASA/Fermi grant NNX1OA057G, and National Science Foundation (NSF) grant AST-0908886. Observations obtained with the Samuel Oschin Telescope at the Palomar Observatory as part of the PTF project, a scientific collaboration between the California Institute of Technology, Columbia University, Las Cumbres Observatory, the Lawrence Berkeley National Laboratory, the National Energy Research Scientific Computing Center, the University of Oxford, and the Weizmann Institute of Science. This publication makes use of data products from the Two Micron All-Sky Survey, which is a joint project of the University of Massachusetts and the Infrared Processing and Analysis Center/California Institute of Technology, funded by the National Aeronautics and Space Administration and the National Science Foundation. This work is based in part on archival data obtained with the Spitzer Space Telescope, which is operated by the Jet Propulsion Laboratory, California Institute of Technology under a contract with NASA. This research has made use of the NASA/IPAC/ NExScI Star and Exoplanet Database, which is operated by the Jet Propulsion Laboratory, California Institute of Technology, under contract with the National Aeronautics and Space Administration. This research has made use of the NASA/IPAC Infrared Science Archive, which is operated by the Jet Propulsion Laboratory, California Institute of Technology, under contract with the National Aeronautics and Space Administration. This research has made use of the VizieR catalogue access tool, CDS, Strasbourg, France. Support for this work was provided by an award issued by JPL/Caltech.

Facilities: PO:1.2m (PTF), Spitzer

\section{REFERENCES}

Aigrain, S., Hodgkin, S., Irwin, J., Hebb, L., Irwin, M., Favata, F., Moraux, E., \& Pont, F. 2007, MNRAS, 375, 29

Armitage, P. J. 2009, in AIP Conf. Ser. 1192, XIII Special Courses at the National Observatory of Rio de Janeiro, ed. F. Roig,

D. Lopes, R. de La Reza, \& V. Ortega (Melville, NY: AIP), 3

Basri, G., et al. 2010, ApJ, 713, L155
Bertin, E. 2006, in ASP Conf. Ser. 351, Astronomical Data Analysis Software and Systems XV, ed. C. Gabriel, C. Arviset, D. Ponz, \& S. Enrique (San Francisco, CA: ASP), 112

Bertin, E., \& Arnouts, S. 1996, A\&AS, 117, 393

Bilir, S., Karataş, Y., Demircan, O., \& Eker, Z. 2005, MNRAS, 357, 497

Bilir, S., et al. 2008, Astron. Nachr., 329, 835

Bouvier, J., et al. 2003, A\&A, 409, 169

Briceño, C., Calvet, N., Hernández, J., Vivas, A. K., Hartmann, L., Downes, J. J., \& Berlind, P. 2005, AJ, 129, 907

Briceño, C., Hartmann, L., Hernández, J., Calvet, N., Vivas, A. K., Furesz, G., \& Szentgyorgyi, A. 2007, ApJ, 661, 1119

Briceño, C., et al. 2001, Science, 291, 93

Brown, A. G. A., Walter, F. M., \& Blaauw, A. 1999, arXiv:astro-ph/9802054v2 Cardelli, J. A., Clayton, G. C., \& Mathis, J. S. 1989, ApJ, 345, 245

Cargile, P. A., Stassun, K. G., \& Mathieu, R. D. 2008, ApJ, 674, 329

Covino, E., Frasca, A., Alcalá, J. M., Paladino, R., \& Sterzik, M. F. 2004, A\&A, 427, 637

Covino, E., et al. 2000, A\&A, 361, L49

de Zeeuw, P. T., Hoogerwerf, R., de Bruijne, J. H. J., Brown, A. G. A., \& Blaauw, A. 1999, AJ, 117, 354

Eggleton, P. P., \& Kisseleva-Eggleton, L. 2006, Ap\&SS, 304, 75

Eker, Z., Bilir, S., Yaz, E., Demircan, O., \& Helvaci, M. 2009, Astron. Nachr., 330,68

Eker, Z., Demircan, O., Bilir, S., \& Karatas, Y. 2006, MNRAS, 373, 1483

Everett, M. E., \& Howell, S. B. 2001, PASP, 113, 1428

Fazio, G. G., et al. 2004, ApJS, 154, 10

Gautier, T. N., III., et al. 2007, ApJ, 667, 527

Gazeas, K., \& Stępień, K. 2008, MNRAS, 390, 1577

Grillmair, C. C., et al. 2010, in ASP Conf. Ser. 434, Astronomical Data Analysis Software and Systems XIX, ed. Y. Mizumoto, I. I. Morita, \& M. Ohishi (Ann Arbor, MI: ASP), 28

Guieu, S., et al. 2010, ApJ, 720, 46

Hebb, L., et al. 2010, A\&A, 522, A37

Herbst, W., Herbst, D. K., Grossman, E. J., \& Weinstein, D. 1994, AJ, 108, 1906 Hillenbrand, L. A. 2008, Phys. Scr. T, 130, 014024

Irwin, J., et al. 2007, MNRAS, 380, 541

Jeffries, R. D., Oliveira, J. M., Naylor, T., Mayne, N. J., \& Littlefair, S. P. 2007, MNRAS, 376, 580

Kenyon, S. J., \& Hartmann, L. 1995, ApJS, 101, 117

Kharchenko, N. V., Piskunov, A. E., Röser, S., Schilbach, E., \& Scholz, R. 2005, A\&A, 440, 403

Kraus, A. L., Craine, E. R., Giampapa, M. S., Scharlach, W. W. G., \& Tucker, R. A. 2007, AJ, 134, 1488

Kraus, A. L., Tucker, R. A., Thompson, M. I., Craine, E. R., \& Hillenbrand, L. A. 2011, ApJ, 728, 48

Lamm, M. H., Bailer-Jones, C. A. L., Mundt, R., Herbst, W., \& Scholz, A. 2004, A\&A, 417, 557

Landolt, A. U. 1992, AJ, 104, 340

Landsman, W. B. 1993, in ASP Conf. Ser. 52, Astronomical Data Analysis Software and Systems II, ed. R. J. Hanisch, R. J. V. Brissenden, \& J. Barnes (San Francisco, CA: ASP), 246

Law, N. M., et al. 2009, PASP, 121, 1395

Law, N. M., et al. 2010, Proc. SPIE, 7735, 7735M

Mathieu, R. D., Baraffe, I., Simon, M., Stassun, K. G., \& White, R. 2007, Protostars and Planets V (Tucson, AZ: Univ. Arizona Press), 411 
McGehee, P. M. 2006, AJ, 131, 2959

Miller, A. A., Irwin, J., Aigrain, S., Hodgkin, S., \& Hebb, L. 2008, MNRAS, 387,349

Monet, D. G., et al. 2003, AJ, 125, 984

Morales-Calderón, M., et al. 2011, ApJ, 733, 50

Plavchan, P., Jura, M., Kirkpatrick, J. D., Cutri, R. M., \& Gallagher, S. C. 2008, ApJS, 175, 191

Pribulla, T., \& Rucinski, S. M. 2006, AJ, 131, 2986

Rau, A., et al. 2009, PASP, 121, 1334

Rieke, G. H., et al. 2004, ApJS, 154, 25

Robin, A. C., Reylé, C., Derriere, S., \& Picaud, S. 2003, A\&A, 409, 523

Roeser, S., Demleitner, M., \& Schilbach, E. 2010, AJ, 139, 2440

Roxburgh, I. W. 1966, ApJ, 143, 111

Rucinski, S. M. 2004, New Astron. Rev., 48, 703

Rucinski, S. M. 2007, MNRAS, 382, 393

Rucinski, S. M., Pribulla, T., \& van Kerkwijk, M. H. 2007, AJ, 134, 2353

Schlegel, D. J., Finkbeiner, D. P., \& Davis, M. 1998, ApJ, 500, 525

Siess, L., Dufour, E., \& Forestini, M. 2000, A\&A, 358, 593
Skrutskie, M. F., et al. 2006, AJ, 131, 1163

Stassun, K. G., Mathieu, R. D., Cargile, P. A., Aarnio, A. N., Stempels, E., \& Geller, A. 2008, Nature, 453, 1079

Stassun, K. G., Mathieu, R. D., \& Valenti, J. A. 2006, Nature, 440, 311

Stassun, K. G., Mathieu, R. D., \& Valenti, J. A. 2007, ApJ, 664, 1154

Stassun, K. G., Mathieu, R. D., Vaz, L. P. R., Stroud, N., \& Vrba, F. J. 2004, ApJS, 151,357

Stauffer, J. R., et al. 2007, ApJS, 172, 663

Stempels, H. C., Hebb, L., Stassun, K. G., Holtzman, J., Dunstone, N., Glowienka, L., \& Frandsen, S. 2008, A\&A, 481, 747

Stępień, K. 1995, MNRAS, 274, 1019

Stępień, K. 2006, Acta Astron., 56, 199

Stetson, P. B. 1987, PASP, 99, 191

Tody, D. 1986, Proc. SPIE, 627, 733

Tody, D. 1993, in ASP Conf. Ser. 52, Astronomical Data Analysis Software and Systems II, ed. R. J. Hanisch, R. J. V. Brissenden, \& J. Barnes (San Francisco, CA: ASP), 173

Tokovinin, A., Thomas, S., Sterzik, M., \& Udry, S. 2006, A\&A, 450, 681

Werner, M. W., et al. 2004, ApJS, 154, 1 